\title{
Non-Fermi Liquid Regimes and Superconductivity in the Low Temperature Phase Diagrams of Strongly Correlated $d$ - and $f$-Electron Materials
}

\author{
M. Brian Maple • Ryan E. Baumbach • \\ Nicholas P. Butch • James J. Hamlin • \\ Marc Janoschek
}

Received: 21 July 2010 / Accepted: 8 August 2010 / Published online: 31 August 2010

(C) The Author(s) 2010. This article is published with open access at Springerlink.com

\begin{abstract}
Standard models for simple metals and insulators often fail for systems based on elements with unstable $d$ - or $f$-electron shells, where strong electronic correlations can generate new and unexpected states of matter. Such a scenario can often be induced when a magnetic phase transition is tuned to absolute zero temperature by an external control parameter such as chemical composition, pressure or magnetic field. At the resulting quantum critical point (QCP), emergent phenomena, such as unconventional superconductivity and novel magnetic phases are frequently observed. The temperature and energy dependences of the physical properties are also found to deviate from expectations for a simple Fermi liquid. This "non-Fermi-liquid" (NFL) behavior is commonly manifested as weak power laws and logarithmic divergences in the physical properties at low temperatures and is often found in a V-shaped region near a QCP, which has become the "classic" QCP phase diagram. However, there is also a growing number of materials where the NFL behavior either occurs far away from the QCP, within an ordered phase, or may not be associated with any putative QCP. Thus, after nearly 20 years of research, it remains unknown whether NFL physics is universal, or if a multitude of unique subclasses exist. In this article, we review research that has primarily been carried out in our laboratory on systems that exhibit NFL behavior that does not conform to the "classic" QCP scenario.
\end{abstract}

Keywords Strongly correlated electrons · Non-Fermi liquid · Quantum criticality

M. Brian Maple (凶) · R.E. Baumbach · J.J. Hamlin · M. Janoschek Department of Physics, University of California, San Diego, La Jolla, CA 92093-0354, USA

e-mail: mbmaple@physics.ucsd.edu

N.P. Butch

Department of Physics and CNAM, University of Maryland, College Park, MD 20742, USA 


\section{Introduction}

A large number of strongly correlated $d$ - and $f$-electron systems have been investigated during the past two decades in which the Fermi liquid paradigm appears to be violated [1-5]. These systems are based on certain intermetallic compounds containing transition metal, lanthanide, or actinide ions such as $\mathrm{Mn}, \mathrm{Fe}, \mathrm{Ce}, \mathrm{Pr}, \mathrm{Sm}, \mathrm{Eu}$, $\mathrm{Yb}$ and $\mathrm{U}$ with partially-filled $d$ - or $f$-electron shells in which the localized $d$ - or $f$-states are hybridized with conduction electron states. The interaction between the magnetic dipole and electric quadrupole moments of the transition metal, lanthanide, or actinide ions and the spins and charges of the conduction electrons generally leads to magnetic or quadrupolar order at sufficiently low temperatures. In these systems, complex phase diagrams of temperature $T$ vs. a control parameter $\delta$, such as chemical composition $x$, pressure $P$, and magnetic field $H$, have been found which contain regions that exhibit non-Fermi liquid (NFL) behavior in the physical properties at low temperature. The NFL behavior is characterized by logarithmic or weak power law divergences in the physical properties at low temperatures $T$ and an effective Fermi temperature that appears in the dynamical susceptibility that is the temperature $T$ itself (i.e., $\omega / T$ scaling). The NFL behavior was first established in chemically substituted $f$-electron compounds containing nonmagnetic substituents $[1,6$, 7] and subsequently observed in stoichiometric $f$-electron compounds [8, 9]. Since these NFL characteristics are found in both chemically substituted and stoichiometric compounds, the underlying physics does not seem to be primarily driven by atomic disorder.

A widely accepted "explanation" of NFL behavior is the classic quantum critical point (QCP) scenario in which the NFL behavior is associated with quantum mechanical fluctuations of the order parameter about the QCP, the critical value $\delta_{c}$ of $\delta$ at which a second order phase transition is suppressed to $0 \mathrm{~K}$. In the QCP scenario, the line describing the second order phase transition and the line that represents the crossover from NFL behavior to Fermi liquid (FL) behavior emanate from the QCP and form a "V-shape" on a plot of temperature vs. control parameter as illustrated in Fig. 1(a). However, there are a large number of $d$ - and $f$-electron systems that do not conform to the QCP scenario; in some systems, the NFL behavior extends far from the QCP into the unordered region or does not even seem to be associated with a readily identifiable QCP (Fig. 1(b)), whereas in other systems, the NFL behavior extends deep into the ordered region. A way of visualizing these situations is in terms of a "line of QCPs" like one would encounter in a chemically substituted system in which the NFL behavior is associated with single ion physics where the "single ion" QCP is independent of composition (as long as interactions between ions do not become so strong as to significantly alter the single ion physics). In some of these systems, the NFL behavior may be attributable to a single ion description such as the Kondo disorder model $[10,11]$ or a multichannel Kondo effect [12-16], whereas in other systems, the NFL behavior may be ascribed to interacting ion models such as the Griffiths phase model [17] that incorporates the Kondo effect and the RKKY interaction in the presence of disorder and anisotropy. On the other hand, the nearly universal recurrence of the $T$-linear electrical resistivity $\rho, \log (T)$ divergence of the specific heat $C$ divided by $T, C / T$ (the electronic specific heat coefficient $\gamma=C / T$ ), non 

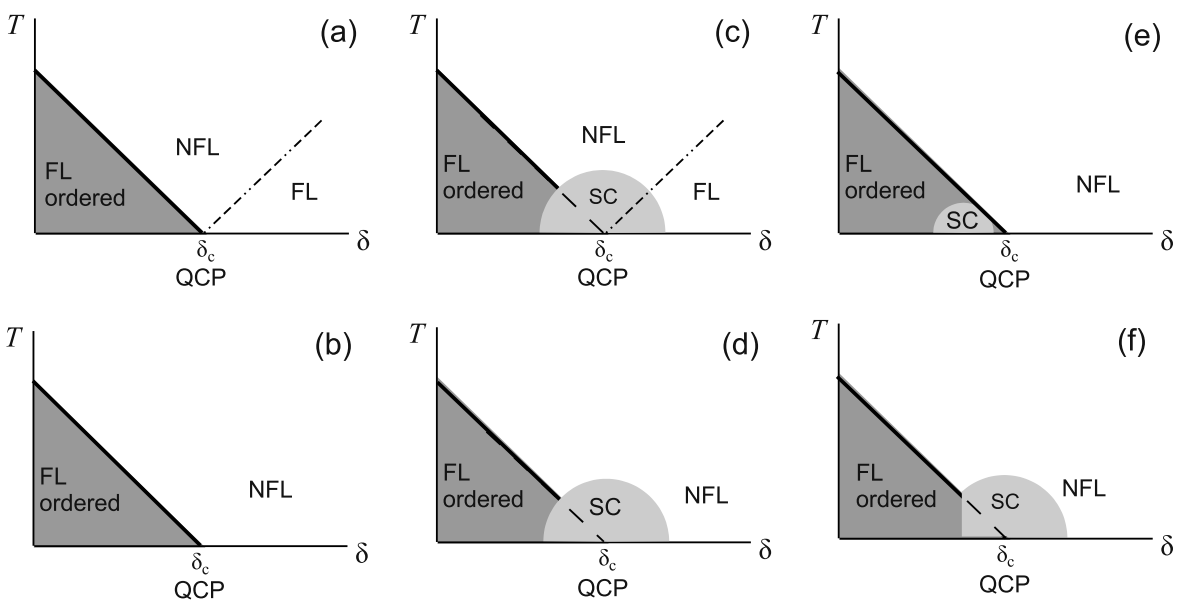

Fig. 1 Schematic temperature $T$ vs. control parameter $\delta$ phase diagram for different NFL scenarios, where $\delta$ is usually chemical composition $x$, pressure $P$ or magnetic field $H$. The ordered state exhibiting FL behavior is suppressed when the control paramater $\delta$ is increased. At the critical value $\delta_{c}$, the order parameter vanishes at a quantum critical point (QCP). The phase diagrams illustrate the salient features of some of the different scenarios. Not every phase diagram has been observed in the exact form in real systems. However, when similar phase diagrams have been reported in the literature, this is noted in the corresponding references. (a) NFL behavior occurs in a narrow V-shaped region above the QCP. For higher values $\delta>\delta_{c}$, FL behavior is recovered. An example of this scenario is $\mathrm{YbRh}_{2} \mathrm{Si}_{2}$, where the control parameter is the magnetic field (Fig. 24) [47, 235, 238]. (b) NFL behavior resides within an extended range of the control parameter $\delta>\delta_{c}$. Examples are $\mathrm{Ce}_{1-x} \mathrm{Y}_{x} \mathrm{RhIn}_{5}$ as a function of Y concentration (Fig. 12) [161] and MnSi as function of pressure (Fig. 13) [179]. (c) The QCP is "protected" by some type of order (usually superconductivity) that develops in a "dome" around the critical value of the control parameter $\delta_{c}$. NFL behavior is observed in a $\mathrm{V}$-shape above the protected phase and FL is recovered for $\delta>\delta_{c}$. A prototypical system for this scenario is CeIn 3 as function of pressure [124]. (d) This situation is similar to (c), but NFL behavior is observed outside the ordered and SCing phases. (e) The SC occurs entirely within the ordered phase. A similar phase diagram is found for $\mathrm{UGe}_{2}$ under pressure, although no NFL behavior is observed and the transition at $\delta_{c}$ is first order (Fig. 15) [207, 219]. (f) SC occurs only outside of the ordered phase. This scenario is realized in CeRhIn 5 as a function of pressure [132]

Curie-Weiss $T$-dependence of the magnetic susceptibility $\chi$, and $\omega / T$ scaling of the imaginary part of the dynamical susceptibility $\chi^{\prime \prime}(\omega, T)$ suggest that there may be a more general picture that encompasses all of these disparate situations that remains to be developed.

Another remarkable phenomenon is the occurrence of some type of order (often superconducting order) that envelops and seemingly "protects" the QCP by removing the large amount of degeneracy associated with the quantum mechanical fluctuations of the order parameter as shown in Figs. 1(c)-1(d). The presence of the degeneracy can be seen in some systems as a peak in the residual resistivity $\rho(0)$ or isotherms of entropy $S(T)$ in the neighborhood of the QCP. In such cases, the new phase that envelops the QCP generally extends to both lower and higher values of $\delta$ than $\delta_{c}$, or, in other words, coexists with both the ordered and the unordered phases. However, there are also cases where the new phase only coexists with either the ordered or unordered phase, and excludes the other. The most striking example of this is superconductivity near magnetic quantum critical points. In many systems, superconductivity coexists with both the magnetically ordered and paramagnetic phases (Figs. 1(c) and 1(d)), 
while for other systems, it coexists with only the magnetically ordered (Fig. 1(e)) or the paramagnetic phase (Fig. 1(f)). The case of superconductivity raises other important issues such as the symmetry of the superconducting order parameter which involves singlet or triplet spin pairing of electrons.

The purpose of this article is to review research that has primarily been carried out in our laboratory during the past two decades on systems that exhibit NFL behavior that does not conform to the classic QCP scenario and the occurrence of superconductivity near QCPs, especially for FM QCPs and quadrupolar QCPs. The systems discussed are primarily strongly correlated $f$-electron systems based on $\mathrm{Ce}, \mathrm{Pr}, \mathrm{Yb}$ and $\mathrm{U}$ ions, although we also discuss related behavior in $d$-electron systems, particularly MnSi and elemental Fe.

\subsection{Outline of this paper}

In this paper, the temperature-control parameter $(T-\delta)$ phase diagrams, where $\delta$ is chemical concentration $x$, pressure $P$ or magnetic field $H$, and NFL characteristics in the electrical resistivity $\rho$, specific heat $C$, and magnetic susceptibility $\chi$ at low temperatures of a number of $f$-electron systems we have investigated in our laboratory are reviewed. Most of these systems do not conform to the simple QCP scenariothe NFL behavior either occurs far away from the QCP, within the ordered phase, or may not be associated with any readily identifiable QCP. These systems include several U-based systems, $M_{1-x} \mathrm{U}_{x} \mathrm{Pd}_{3}(M=\mathrm{Sc}, \mathrm{Y}), \mathrm{U}_{1-x} M_{x} \mathrm{Pd}_{2} \mathrm{Al}_{3}(M=\mathrm{Y}, \mathrm{La}$, $\mathrm{Th}$ ), and $\mathrm{URu}_{2-x} \mathrm{Re}_{x} \mathrm{Si}_{2}$, a few Ce-based systems, $\mathrm{CeRh}_{1-x} \mathrm{Co}_{x} \mathrm{In}_{5}, \mathrm{Ce}_{1-x} \mathrm{Y}_{x} \mathrm{RhIn}_{5}$, $\mathrm{Ce}_{1-x} R_{x} \mathrm{CoIn}_{5}$, and a newly discovered Yb-based NFL system $\mathrm{Yb}_{2} \mathrm{Fe}_{12} \mathrm{P}_{7}$.

We also discuss the temperature-pressure $(T-P)$ and chemical composition $(T-x)$ phase diagrams of various $f$-electron compounds that exhibit superconductivity in the vicinity of magnetic quantum critical points that are accessed through the application of an external pressure. These include the antiferromagnetic compounds $\mathrm{CeIn}_{3}[9,18], \mathrm{CeRhIn}_{5}$ [19], and the ferromagnetic compounds $\mathrm{UGe}_{2}[20,21]$, URhGe [22, 23], and UCoGe [24-26].

A brief description is given of the heavy femion compound $\mathrm{PrOs}_{4} \mathrm{Sb}_{12}$ in which unconventional superconductivity occurs near an antiferroquadrupolar ordered state and may be mediated by quadrupolar fluctuations [27, 28].

Finally, we compare the NFL behavior and phase diagrams for the $f$-electron materials discussed in this article with the NFL behavior observed in $d$-electron systems such as MnSi [29] and elemental Fe [30, 31].

\subsection{Non-Fermi Liquid Characteristics}

For many of the $f$-electron systems, the electrical resistivity $\rho(T)$, specific heat $C(T)$, magnetic susceptibility $\chi(T)$, and imaginary part of the dynamical magnetic susceptibility $\chi^{\prime \prime}(\omega, T)$ have the following NFL temperature dependences for $T \ll T_{0}$ :

(i) $\rho(T) \sim \pm\left(T / T_{0}\right)^{n}$ where $n \approx 1-1.5$ ( $n \sim 0.5$ has also been observed);

(ii) $C(T) / T \sim\left(-1 / T_{0}\right) \ln \left(T / T_{0}\right)$, or $\sim T^{-1+\lambda}(\lambda \sim 0.7-0.8)$; 
(iii) $\chi(T) \sim 1-\left(T / T_{0}\right)^{1 / 2}, \sim\left(-1 / T_{0}\right) \ln \left(T / T_{0}\right), T^{-1+\lambda}(\lambda \sim 0.7-0.8)$, or $C /\left(T^{\alpha}-\right.$ $\Theta)$;

(iv) $\chi^{\prime \prime}(\omega, T)$ scales as $\omega / T$, where $\omega$ is the frequency.

A detailed discussion of these $T$-dependences follows below. In several of the $f$ electron systems, the characteristic temperature $T_{0}$ can be identified with the Kondo temperature $T_{K}$. These new NFL $f$-electron materials can be compared with "conventional" heavy fermion $f$-electron compounds, such as $\mathrm{CeAl}_{3}$ and $\mathrm{UPt}_{3}$, which behave as Fermi liquids [32], in spite of the strong electron-electron interactions that renormalize the electron mass by $\sim 10^{2}-10^{3}$ (or, equivalently, the effective Fermi temperature $T_{F}$ is low, $\left.\sim 1-10 \mathrm{~K}\right)$. Here, the temperature and frequency dependences of the physical properties scale with $T_{F}$. The quantities $\rho(T), C(T), \chi(T)$, and $\chi^{\prime \prime}(T)$ have the following familiar FL forms for $T \ll T_{F}$ :

(i) $\rho(T) \sim \pm\left(T / T_{F}\right)^{2}$;

(ii) $C(T) / T \sim \gamma_{0}$, where $\gamma_{0}$ can be as large as $\sim$ several $\mathrm{J} / \mathrm{mol}-\mathrm{K}^{2}$ !;

(iii) $\chi(T) \sim \chi_{0}$, such that $\chi_{0} / \gamma_{0} \sim 1$;

(iv) $\chi^{\prime \prime}(\omega, T)$ scales as $\omega / T_{F}$.

\subsection{Issues Concerning Characterization of NFL Behavior}

The electrical resistivity of the NFL systems displays power law behavior of the form $\rho(T) \sim \rho_{0} \pm A T^{n}$ where $n$ is often close to 1 or $3 / 2$ and in a few instances as low as $1 / 2[33,34]$. In many cases, the power law dependence can extend over several decades in temperature. A common method of analyzing the resistivity is to plot the $\rho(T)$ data as $\ln \left[\rho(T)-\rho_{0}\right]$ vs. $\ln T$ and to adjust $\rho_{0}$ to give the best fit of the data by a straight line over the largest range of temperature with emphasis on the data at the lowest temperatures [6]. Such a straight line has the form $\ln \left[\rho(T)-\rho_{0}\right]=$ $\ln A+n \ln T$, from which $\rho_{0}, A$ and $n$ can be determined. In performing such an analysis, it is most meaningful that it be performed on cubic materials if they are in the form of polycrystals; if the compounds are not cubic, it is preferable to work with single crystal specimens, if possible, to avoid obtaining a polycrystalline average over the different grains with varying orientations with respect to the current direction in the specimen.

One of the most striking types of NFL behavior, which is nearly universal, is found in the specific heat $C(T)$, which can generally be described by a logarithmic or weak power law divergence in temperature $T$ with decreasing $T$ of $C(T) / T[1,2,6,7$, $35]$. In some cases, this behavior crosses over into a more rapid divergence with decreasing $T$. This more rapid dependence has been attributed to magnetic ordering at lower $T$, lifting the degeneracy of the NFL ground state. For example, the quadrupolar Kondo effect (an electric quadrupole version of the spin 1/2, two channel magnetic Kondo effect) has a logarithmic divergence of $C(T) / T$ with decreasing $T$ and a residual entropy at $T=0 \mathrm{~K}$ of $(1 / 2) k_{B} \ln 2$ per ion with an electric quadrupole or magnetic dipole moment. If quadrupolar or magnetic ordering occurs at lower $T$, this entropy has to be removed, which will result in a faster increase of $C(T) / T$ with decreasing $T$. In zero magnetic field, the specific heat is a reliable measure of NFL behavior for polycrystalline samples. However, in finite magnetic field, it is necessary to work with single crystals to get meaningful results and avoid a polycrystalline 
average of the response from different grains with varying orientations with respect to the applied magnetic field.

The magnetic susceptibility of systems that exhibit NFL behavior seems to have many different $T$-dependences. This may be due to magnetic impurities which introduce Brillouin contributions to the magnetization $M$ that become large at low temperatures and saturate at large values of $\mu H / k_{B} T$, where $\mu$ is the magnetic moment of the magnetic impurity ion. On the other hand, $M(H)$ may have negative curvature that is intrinsic and due to a crossover from NFL to FL behavior. As a result, it is difficult to determine whether the magnetization with negative curvature sometimes found for NFL systems is intrinsic or associated with contributions from magnetic impurities. This yields uncertainties in how to analyze the magnetization measurements; i.e., whether to determine $\chi(T)$ from the low field susceptibility or correct the magnetization for the possible presence of magnetic impurities as described in other publications $[6,36]$.

\subsection{Routes to Non-Fermi Liquid Behavior}

Experiments on a variety of $f$-electron systems suggest that there are two general routes to NFL behavior involving interactions of the charges or spins of the conduction electrons with (1) unordered (single ion effect) and (2) ordered electric quadrupole or magnetic dipole moments of the transition metal, lanthanide or actinide ions. Case (1) appears to be related to an unconventional Kondo effect, while case (2) is associated with order parameter fluctuations in the vicinity of a second order magnetic (or, possibly, quadrupolar) phase transition that has been suppressed to zero temperature (quantum critical point QCP) or an effective field deep in the ordered state. Theoretical models based on single ion physics include a multichannel Kondo effect, of either magnetic or electric (quadrupolar) origin [12-16] and a single channel Kondo effect with a distribution of Kondo temperatures due to chemical disorder (referred to as the "Kondo disorder" model) $[10,11]$. Theoretical models that incorporate interionic interactions include fluctuations of an order parameter in the vicinity of a second order phase transition at $0 \mathrm{~K}$ (quantum critical point model) [37-44], an inhomogeneous Griffiths phase [17], or ferromagnetic order [45]. The Griffiths phase consists of magnetic clusters in a paramagnetic phase and forms as a result of the competition between the Kondo effect and the Ruderman-Kittel-Kasuya-Yosida (RKKY) interaction in the presence of magnetic anisotropy and disorder. Most of the chemically substituted systems in which NFL behavior is found have complex phase diagrams containing a rich variety of strongly correlated electron phenomena; e.g., magnetic order, quadrupolar order, spin glass freezing, Kondo effect coupled with low temperature NFL behavior, heavy fermion behavior, and superconductivity. The proximity of the glassy or long-range ordered magnetic phases to the phases with Kondo and low temperature NFL behavior has made it difficult to distinguish between single ion or cooperative effects as the source of the NFL behavior.

The QCP model has been widely applied to situations where a second order phase transition, usually antiferromagnetic (AFM), is suppressed to $T=0 \mathrm{~K}$ by a control parameter such as chemical composition $(x)$, pressure $(P)$, or magnetic field $(H)$, terminating in a QCP at $T=0 \mathrm{~K}$. Order parameter fluctuations are manifested as NFL 
behavior at temperatures above the QCP and a line emanating from the QCP delineates a gradual crossover from NFL to FL behavior at lower $T$ and higher values of the control parameter. Extensive investigations of several prototypical systems (e.g., $\mathrm{CeCu}_{6-x} \mathrm{Au}_{x}$ [46] and $\mathrm{YbRh}_{2} \mathrm{Si}_{2}$ [47]) have been analyzed within the context of the QCP model and support the predicted "V-shaped" temperature-control parameter phase diagram.

\section{$2 \mathbf{Y}_{1-x} \mathbf{U}_{x} \mathbf{P d}_{3}$}

An experimental investigation of the $\mathrm{Y}_{1-x} \mathrm{U}_{x} \mathrm{Pd}_{3}$ system, carried out in our laboratory in 1990, revealed an unconventional Kondo effect with concomitant NFL behavior at low temperatures for $\mathrm{U}$ concentrations $x$ in the range $0<x \leq 0.2[6,48]$. The NFL characteristics included a linear $T$-dependence of $\rho$, a logarithmic in $T$ divergence of $C / T$, and a non-Curie Weiss $\chi$ that varies as $T^{1 / 2}$. Interestingly, these NFL signatures were later observed in many other $d$ - and $f$-electron systems [1, $2,4,5]$, including both chemically substituted and stoichiometric compounds. The low temperature-composition $(T-x)$ phase diagram of the $\mathrm{Y}_{1-x} \mathrm{U}_{x} \mathrm{Pd}_{3}$ system is shown in Fig. 2 [49]. Within the $U$ concentration interval $0<x \leq 0.5$, where $\mathrm{Y}_{1-x} \mathrm{U}_{x} \mathrm{Pd}_{3}$ crystallizes in the cubic $\mathrm{Cu}_{3} \mathrm{Au}$ structure, three different ground states are found: non-Fermi liquid (NFL) behavior $(0<x \lesssim 0.2)$, spin glass (SG) freezing $(0.2 \lesssim x \lesssim 0.42)$, and long range antiferromagnetic (AFM) order $(0.42 \lesssim x \lesssim 0.54)$. The rapid decrease of the Kondo temperature $T_{K}$ with $x$, indicated in Fig. 2, has been attributed to a 'Fermi level tuning' phenomenon in which the $\mathrm{U}^{4+} 5 f$ binding energy $\varepsilon_{f}=E_{F}-E_{f}$, where $E_{F}$ is the Fermi energy and $E_{f}$ is the energy of the $\mathrm{U}^{4+} 5 \mathrm{f}$ state, increases by $1 \mathrm{eV}$ as $x$ increases from 0 to $1[1,50]$. The increase of $\varepsilon_{f}$ with $x$ was discovered in photoemission studies of $\mathrm{Y}_{1-x} \mathrm{U}_{x} \mathrm{Pd}_{3}$ [51] and can be understood in terms of the increase of $E_{F}$ with $x$ as tetravalent $\mathrm{U}$ is substituted for trivalent $\mathrm{Y}$. The nearly linear increase of $\varepsilon_{f}$ with $x$ should cause a rapid decrease in $T_{K}$ since

$$
\left.T_{K} \sim T_{F} \exp \left[-1 / N\left(E_{F}\right)|\boldsymbol{J}|\right)\right] \sim T_{F} \exp \left[-\varepsilon_{f} / N\left(E_{F}\right)\left\langle V_{k f}^{2}\right\rangle\right]
$$

where $T_{F}$ is the Fermi temperature, $N\left(E_{F}\right)$ is the density of states at $E_{F}$, $J \sim-\left\langle V_{k f}^{2}\right\rangle / \varepsilon_{F}$ is the exchange interaction parameter, and $V_{k f}$ is the hybridization matrix element that characterizes the admixture of the localized $5 f$ states of $U$ and the conduction electron states. Assuming that $\varepsilon_{f}$ increases linearly with $x$, it follows from (1) that $\ln \left(T_{K}\right)$ decreases linearly with $x$; i.e.,

$$
\ln \left(T_{K}\right)=\ln \left(T_{F}\right)-\varepsilon_{f} / N\left(E_{F}\right)\left\langle V_{k f}^{2}\right\rangle \propto-\varepsilon_{f} \propto-x
$$

The linear decrease of $\ln \left(T_{K}\right)$ with $x$ is borne out by the experimental $\ln \left(T_{K}\right)$ vs. $x$ data for $\mathrm{Y}_{1-x} \mathrm{U}_{x} \mathrm{Pd}_{3}$ plotted in Fig. 2.

An analysis of the NFL characteristics in the U contributions to $\rho(T), C(T)$, and $\chi(T)$, the quantities $\Delta \rho(T), \Delta C(T)$, and $\Delta \chi(T)$, respectively, indicate that these features scale with $\mathrm{U}$ concentration $x$ and Kondo temperature $T_{K}$, where $T_{K}$ is inferred from the high temperature behavior of $\rho(T)$ and $\chi(T)$, at least over the range $0.1 \leq x \leq 0.2$. It has proven to be difficult to test the scaling of $\Delta \rho(T), \Delta C(T)$, and 

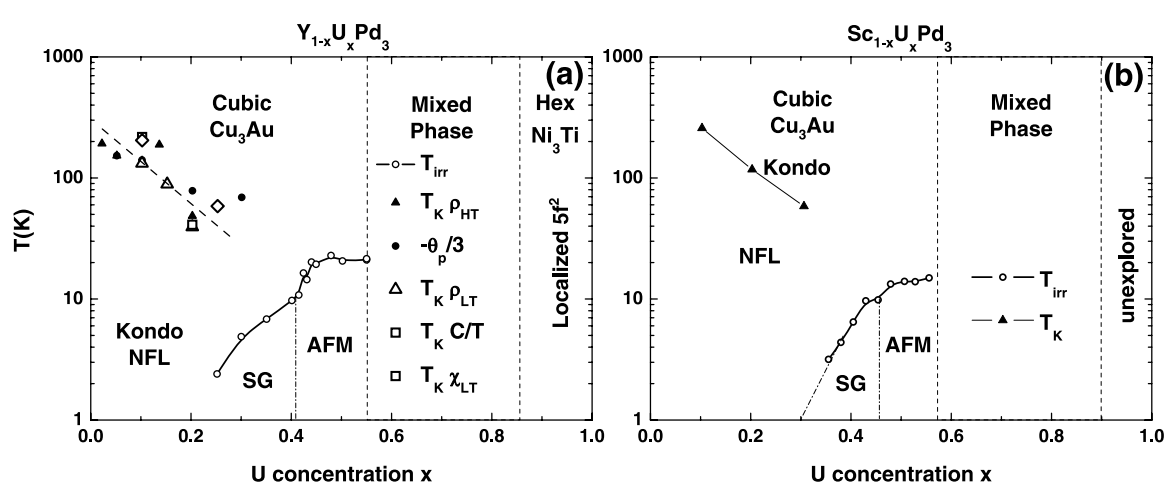

Fig. 2 Temperature-composition $(T-x)$ phase diagrams with a logarithmic temperature scale for the $\mathrm{Y}_{1-x} \mathrm{U}_{x} \mathrm{Pd}_{3}$ (left panel) and $\mathrm{Sc}_{1-x} \mathrm{U}_{x} \mathrm{Pd}_{3}$ (right panel) systems. Antiferromagnetic (AFM), spin glass (SG), Kondo, and non-Fermi liquid (NFL) regimes are identified in the figures. The meanings of the symbols are: $T_{\text {irr }}$-irreversibility temperature; $T_{K}$-Kondo temperature; $\Theta_{p}$-Curie-Weiss temperature; $\rho$-electrical resistivity; $C$-specific heat; $\chi$-magnetic susceptibility; the subscripts HT and LT refer to measurements made at high and low temperatures, respectively. After Refs. [49, 52]

$\Delta \chi(T)$ to lower values of $x$. This is because the NFL features weaken rapidly with decreasing $x$ since their magnitudes are proportional to $x$ and their gradients decrease with increasing $T_{K}$ (decreasing $x$ ). Examples of the striking NFL characteristics in $\Delta \rho(T), \Delta C(T)$, and $\Delta \chi(T)$ found in the $\mathrm{Y}_{1-x} \mathrm{U}_{x} \mathrm{Pd}_{3}$ system at low temperature for a specimen with $x=0.2$ are shown in Figs. 3(a), 3(b), and 3(c), respectively.

The $\Delta \rho(T)$ data displayed in Fig. 3(a) have been fitted by the relation $\Delta \rho(T) /$ $\Delta \rho(0)=1-a\left(T / T_{K}\right)^{n}$, where $\rho(0)$ and $n$ are adjustable fitting parameters. Since the best fit yields the value $n=1.1 \pm 0.1$, it was concluded that the $\Delta \rho(T)$ data are consistent with the expression

$$
\Delta \rho(T) / \Delta \rho(0)=1-a\left(T / T_{K}\right)
$$

The $\Delta C(T) / T$ data shown in Fig. 3(b) can be described well by an expression of the form

$$
\Delta C(T) / T=\left(-b R / T_{K}\right) \ln \left[b^{\prime}\left(T / T_{K}\right)\right]
$$

in the range $0.3 \mathrm{~K}<T \leq 10 \mathrm{~K}$, but deviate from it below $0.3 \mathrm{~K}$. Equation (4) has the form predicted by the two-channel spin-1/2 Kondo model which yields specific values for the contants $b$ and $b^{\prime}$. Within the context of the two-channel spin-1/2 Kondo model, the upturn in $\Delta C(T) / T$ could be due to a lifting of the degeneracy of a $\mathrm{U}^{4+}$ doublet ground state by an exchange field, which could remove the residual $(R / 2) \ln (2)$ entropy. The $\Delta \chi(T)$ data in Fig. 3(c) can be fitted between $0.6 \mathrm{~K}$ and $40 \mathrm{~K}$ by an expression of the form

$$
\Delta \chi(T) / \Delta \chi(0)=1-\chi\left(T / T_{K}\right)^{1 / 2}
$$

after the magnetization $M(H, T)$ data have been corrected by removing a nonlinear contribution that scales with $H /\left(T-\Theta_{p}\right)$ and was assumed to be due to magnetic 

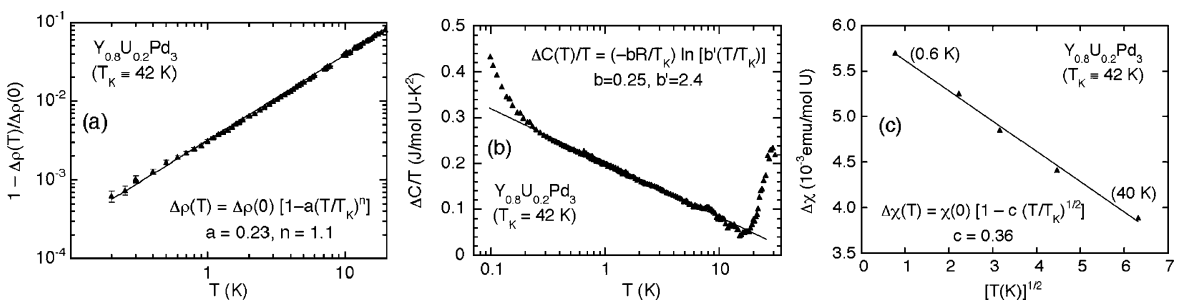

Fig. 3 The $\mathrm{U}$ contribution to the electrical resistivity, $\Delta \rho$, specific heat, $\Delta C$, and magnetic susceptibility, $\Delta \chi$, of $\mathrm{Y}_{0.8} \mathrm{U}_{0.2} \mathrm{Pd}_{3}$, plotted as (a) $\log [1-\Delta \rho(T) / \Delta \rho(0)]$ vs. $\log T$, (b) $\Delta C(T) / T$ vs. $\log T$, and (c) $\Delta \chi(T)$ vs. $T^{1 / 2}$. After Ref. [49]

impurities [1]. The constants $a, b, b^{\prime}$ and $c$ in (3)-(5) have values of the order of unity and were determined from an analysis described in Ref. [49].

The scaling of $\Delta \rho(T), \Delta C(T)$, and $\Delta \chi(T)$ with $x$ and $T_{K}$ suggests that the NFL behavior at low temperatures is a single ion phenomenon that is associated with the Kondo effect observed at higher temperatures. It is noteworthy that some of the NFL features are, in fact, consistent with the predictions of the single ion quadrupolar Kondo model, the electric analogue of the magnetic two-channel spin-1/2 Kondo model. According to this model [15], the electrical resistivity $\Delta \rho(T)$ should vary as

$$
\Delta \rho(T) / \Delta \rho(0)=1-a\left(T / T_{K}\right)^{1 / 2},
$$

a result that is clearly at variance with the experimentally observed linear $T$-dependence described by (3). On the other hand, the quadrupolar Kondo model predicts that the specific heat $\Delta C(T)[13,53]$ and the magnetic susceptibility $\Delta \chi(T)$ [54] have the same forms as (4) and (5), respectively, both of which are consistent with experiment $[1,2,50]$. Furthermore, the applicability of the quadrupolar Kondo model to the $\mathrm{Y}_{1-x} \mathrm{U}_{x} \mathrm{Pd}_{3}$ system requires that the ground state of $\mathrm{U}^{4+}$ in the cubic crystal CEF be the $\Gamma_{3}$ nonmagnetic doublet that carries an electric quadrupole moment. In a cubic CEF, the nine-fold degenerate $J=4$ Hund's rule multiplet of $\mathrm{U}^{4+}$ is split into $\Gamma_{4}$ and $\Gamma_{5}$ triplets, a $\Gamma_{1}$ singlet, and a $\Gamma_{3}$ nonmagnetic doublet.

Several inelastic neutron scattering (INS) experiments have been carried out to determine the ground state of $\mathrm{U}^{4+}$ in the $\mathrm{Y}_{1-x} \mathrm{U}_{x} \mathrm{Pd}_{3}$ system [55-57]. However, the results are not consistent with one another and further research will be required to resolve this issue. From INS measurements on $\mathrm{Y}_{0.8} \mathrm{U}_{0.2} \mathrm{Pd}_{3}$, Mook et al. [55] inferred that the $\mathrm{U}^{4+}$ ground state is the $\Gamma_{3}$ nonmagnetic doublet. Based on polarized INS studies of $\mathrm{Y}_{1-x} \mathrm{U}_{x} \mathrm{Pd}_{3}$ with $x=0.2$ and 0.45 , Dai et al. [56] concluded that the $\mathrm{U}^{4+}$ ground state is the $\Gamma_{5}$ triplet. From INS measurements on $\mathrm{Y}_{1-x} \mathrm{U}_{x} \mathrm{Pd}_{3}$ with $x=0.2$, $0.28,0.37$, and 0.45 , Bull et al. [57] deduced that the $\mathrm{U}^{4+}$ ground state is the $\Gamma_{3}$ doublet with a low lying $\Gamma_{5}$ triplet first excited state. Furthermore, Bull et al. observed that the low energy excitation moves towards zero energy transfer with decreasing $U$ concentration, leading to a ground state in which the $\Gamma_{3}$ doublet and $\Gamma_{5}$ triplet states become degenerate at $x=0.2$.

It should be noted that the $\mathrm{U}$ concentration range within which NFL behavior in the $\mathrm{Y}_{1-x} \mathrm{U}_{x} \mathrm{Pd}_{3}$ system is observed $(0<x \lesssim 0.2)$ is contiguous with the region in which spin glass freezing occurs. This suggests that the NFL behavior in the physical 
properties at low temperature could instead be associated with a magnetic quantum critical point at $x \approx 0.2$. Andraka and Tsvelik [39], who performed measurements of $\rho, C$, and $M$ as a function of $T$ and $H$ on a $\mathrm{Y}_{1-x} \mathrm{U}_{x} \mathrm{Pd}_{3}$ specimen of composition $x=0.2$, concluded from the scaling behavior of $C(T, H)$ and $M(T, H)$ with $H / T$ that the NFL behavior was associated with a second order phase transition at $T=0 \mathrm{~K}$. An interesting possibility is that the NFL behavior may originate from both an unconventional Kondo effect, possibly quadrupolar in character, and order parameter fluctuations associated with a magnetic quantum critical point. On the other hand, interactions between the $\mathrm{U}$ ions could modify the $T$-dependence of $\rho$ so that it varies as $T$ rather than $T^{1 / 2}$, as predicted by the impurity quadrupolar Kondo model.

Motivated by evidence for a quadrupolar Kondo effect (QKE) in $\mathrm{Y}_{1-x} \mathrm{U}_{x} \mathrm{Pd}_{3}[6$, 49], studies of other $M_{1-x} \mathrm{U}_{x} \mathrm{Pd}_{3}$ systems, where $M$ is trivalent or tetravalent, were undertaken [49]. Among the systems investigated, only $\mathrm{Sc}_{1-x} \mathrm{U}_{x} \mathrm{Pd}_{3}$ was found to display NFL behavior [58]. Given that Sc and Y are isoelectronic, this similarity may not seem surprising; however, $\mathrm{La}$ is also isoelectronic with $\mathrm{Sc}$ and $\mathrm{Y}$, but $\mathrm{La}_{1-x} \mathrm{U}_{x} \mathrm{Pd}_{3}$ does not display Kondo or NFL behavior $[49,52]$. The $T-x$ phase diagrams of the $\mathrm{Y}_{1-x} \mathrm{U}_{x} \mathrm{Pd}_{3}$ and $\mathrm{Sc}_{1-x} \mathrm{U}_{x} \mathrm{Pd}_{3}$ systems are compared in Fig. 2, while the $\mathrm{U}$ contributions to the electrical resistivity, $\Delta \rho(T)$, specific heat, $\Delta C(T)$, and magnetic susceptibility, $\Delta \chi(T)$, for the compounds $\mathrm{Y}_{0.8} \mathrm{U}_{0.2} \mathrm{Pd}_{3}$ and $\mathrm{Sc}_{0.7} \mathrm{U}_{0.3} \mathrm{Pd}_{3}$ are displayed in Figs. 4(a), 4(b), and 4(c), respectively. For low values of $x$, the physical phenomena are consistent with single-ion Kondo behavior. Because $\mathrm{Sc}^{3+}$ is smaller than $\mathrm{Y}^{3+}$, the magnitude of the localized $5 \mathrm{f}$-itinerant electron exchange interaction is greater in $\mathrm{Sc}_{1-x} \mathrm{U}_{x} \mathrm{Pd}_{3}$, and thus $T_{K}$ is larger for a given value of $x$. As shown in Fig. 4, the $T$-dependences of the low temperature NFL electronic properties and the values of $T_{K}$ of $\mathrm{Y}_{0.8} \mathrm{U}_{0.2} \mathrm{Pd}_{3}$ and $\mathrm{Sc}_{0.7} \mathrm{U}_{0.3} \mathrm{Pd}_{3}$ are nearly identical. The similar values of $T_{K}$ for the two compounds can be explained in terms of Fermi level tuning [52], discussed above.
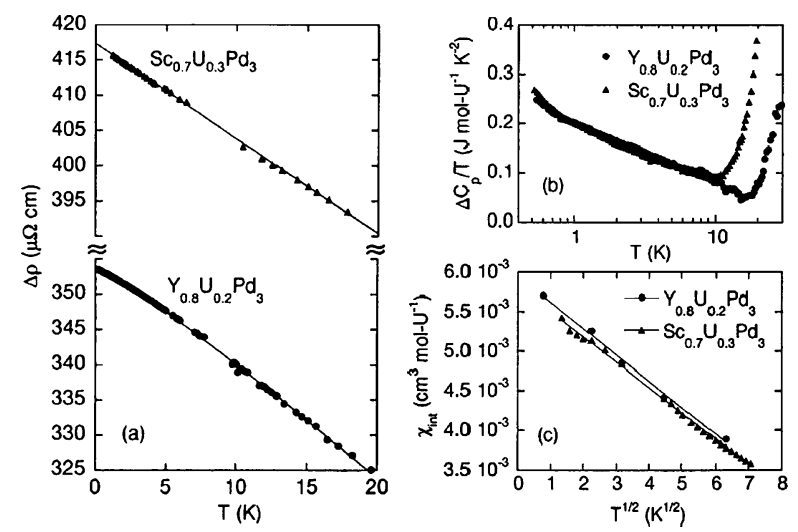

Fig. 4 (a) $\mathrm{U}$ contribution to the electrical resistivity, $\Delta \rho$, vs. $T$ for $\mathrm{Sc}_{0.7} \mathrm{U}_{0.3} \mathrm{Pd}_{3}$ and $\mathrm{Y}_{0.8} \mathrm{U}_{0.2} \mathrm{Pd}_{3}$. Solid lines are fits of (2) to the data with $n=1.0$ and $T_{K}=72 \mathrm{~K}$ for $\mathrm{Sc}_{0.7} \mathrm{U}_{0.3} \mathrm{Pd}_{3}$ and $n=1.1$ and $T_{K}=49 \mathrm{~K}$ for $\mathrm{Y}_{0.8} \mathrm{U}_{0.2} \mathrm{Pd}_{3}$. (b) $\mathrm{U}$ contribution to the electronic specific heat coefficient, $\Delta C_{p} / T$, vs. $T$ on a semilogarithmic scale. (c) Intrinsic magnetic susceptibility, $\chi_{\text {int }}, v s . T^{1 / 2}$. Solid lines are fits of (5) to the data with $T_{K}=43 \mathrm{~K}$ for $\mathrm{Sc}_{0.7} \mathrm{U}_{0.3} \mathrm{Pd}_{3}$ and $T_{K}=42 \mathrm{~K}$ for $\mathrm{Y}_{0.8} \mathrm{U}_{0.2} \mathrm{Pd}_{3}$. After Ref. [52] 
There has been some debate about whether the NFL behavior in the $\mathrm{Y}_{1-x} \mathrm{U}_{x} \mathrm{Pd}_{3}$ system is an intrinsic electronic property or an extrinsic effect associated with metallurgical inhomogeneity that several groups have reported is present in their samples of $\mathrm{Y}_{1-x} \mathrm{U}_{x} \mathrm{Pd}_{3}[59,60]$. Whereas $\mathrm{U}$ is inhomogeneously distributed on a micron length scale in the $\mathrm{Y}_{1-x} \mathrm{U}_{x} \mathrm{Pd}_{3}$ system, $\mathrm{U}$ was found to be nearly homogeneously distributed in the $\mathrm{Sc}_{1-x} \mathrm{U}_{x} \mathrm{Pd}_{3}$ system [52]. However, in spite of the difference in their metallurgical properties, the $\mathrm{Y}_{1-x} \mathrm{U}_{x} \mathrm{Pd}_{3}$ and $\mathrm{Sc}_{1-x} \mathrm{U}_{x} \mathrm{Pd}_{3}$ systems have very similar $T-x$ phase diagrams and NFL characteristics in their low temperature physical properties. These results suggest that the observed NFL behavior in the $\mathrm{Y}_{1-x} \mathrm{U}_{x} \mathrm{Pd}_{3}$ and $\mathrm{Sc}_{1-x} \mathrm{U}_{x} \mathrm{Pd}_{3}$ systems is intrinsic, and not a result of metallurgical inhomogeneity.

Interestingly, the electronic specific heat in $\mathrm{Sc}_{1-x} \mathrm{U}_{x} \mathrm{Pd}_{3}$ was found to follow the form of (2) for $T$ above the SG transition, while for $0.05 \mathrm{~K}<T<2.5 \mathrm{~K}$, the electrical resistivity follows $\Delta \rho(T) \sim 1-a\left(T / T_{K}\right)^{n}$, where $n$ ranges from 1.3 at $x=0.2$ to 0.5 at $x=0.35$ [33]. Surprisingly, for concentrations in the vicinity of the onset of $\mathrm{SG}$ freezing, the low- $T$ resistivity is indeed consistent with the QKE. However, since the sublinear $T$-dependence of $\rho$ of $\mathrm{Sc}_{1-x} \mathrm{U}_{x} \mathrm{Pd}_{3}$ occurs in the region of spin glass ordering, its origin may be at least partially associated with the spin glass phase. It is also interesting to note that sublinear $T$-dependences of $\rho$ have been observed in the NFL compound $\mathrm{CeCoIn}_{5}$ with various lanthanide substituents [34].

Inelastic neutron scattering experiments on $\mathrm{Sc}_{1-x} \mathrm{U}_{x} \mathrm{Pd}_{3}$ [61] reveal excitations in the $x=0.35$ compound that are broad in energy $\hbar \omega, T$-independent, and wave vector $q$-independent, and consistent with a single-ion scenario. Furthermore, the imaginary part of the dynamical susceptibility $\chi^{\prime \prime}(q, \omega, T)$ scales with $\omega / T$ for all values of $q$, $\omega$, and $T$ studied with a scaling exponent $\alpha=1 / 5$, indicating that temperature is the only relevant energy scale. Since the $\omega / T$ scaling is found in a sample that is close to the SG QCP, it is tempting to ascribe the $\omega / T$ scaling of $\chi^{\prime \prime}(\omega, T)$ with $\alpha=1 / 5$ to magnetic fluctuations associated with the spin glass (SG) QCP. Because similar scaling has been observed in $\mathrm{UCu}_{5-x} \mathrm{Pd}_{x}(\alpha=1 / 3)[62,63]$ and $\mathrm{CeCu}_{6-x} \mathrm{Au}_{x}(\alpha=$ 0.75) [64], both of which have AFM QCPs, $\mathrm{URu}_{2-x} \mathrm{Re}_{x} \mathrm{Si}_{2}(\alpha$ ranging from 0.2 at $x=0.2$ to 0.5 at $x=0.6$ ), which has a FM QCP [65], it is possible that the NFL behavior in all of these systems is tied to quantum criticality, specifically related to local moment fluctuations, regardless of the exact nature of the magnetically ordered phase.

\section{$3 \mathrm{U}_{1-x} \mathrm{M}_{x} \operatorname{Pd}_{2} \mathrm{Al}_{3}(M=\mathrm{Th}, \mathrm{Y}, \mathrm{La})$}

Three systems that exhibit NFL behavior at low temperatures, derived by substituting different elements (Th, Y, La) into the same heavy fermion parent compound, $\mathrm{UPd}_{2} \mathrm{Al}_{3}$, have been extensively studied in our laboratory. The NFL behavior in these three systems, $\mathrm{U}_{1-x} M_{x} \mathrm{Pd}_{2} \mathrm{Al}_{3}(M=\mathrm{Th}, \mathrm{Y}, \mathrm{La})$, apparently originates from two different mechanisms: an unconventional Kondo effect $(M=\mathrm{Th})$ and fluctuations of an order parameter in the vicinity of an SG QCP $(M=\mathrm{Y}, \mathrm{La})$. The parent compound $\mathrm{UPd}_{2} \mathrm{Al}_{3}$ has a moderately large electron effective mass $m^{*} \approx 50 m_{e}$, inferred from an electronic specific heat coefficient $\gamma \approx 140 \mathrm{~mJ} / \mathrm{mol} \mathrm{K}^{2}$ [66], and exhibits the coexistence of AFM order with a Néel temperature $T_{N}=14.6 \mathrm{~K}$ and superconductivity 
with $T_{c} \sim 2 \mathrm{~K}$. The AFM structure consists of an AFM stacking along the $c$-axis of FM planes with relatively large ordered moments of $\sim 0.85 \mu_{B}$ lying in the hexagonal basal plane [67]. In the following discussion, we briefly describe the $T-x$ phase diagrams and NFL behavior observed in the Th, Y, and La substituted $\mathrm{UPd}_{2} \mathrm{Al}_{3}$ based systems.

The $T-x$ phase diagram of the $\mathrm{U}_{1-x} \mathrm{Th}_{x} \mathrm{Pd}_{2} \mathrm{Al}_{3}$ system is shown in Fig. 5(a). With increasing Th concentration $x$ below $\sim 0.2, T_{N}$ and $T_{c}$ decrease only slightly [2, 68], whereas the features in $\rho(T), C(T)$, and $\chi(T)$ associated with the AFM and superconducting transitions are suppressed rapidly for $x$ below $\sim 0.2$ [2]. The small decrease of $T_{N}$ and $T_{c}$ with $x$ suggests that $\mathrm{U}$ has the same valence as $\mathrm{Th}(4+)$ in $\mathrm{U}_{1-x} \mathrm{Th}_{x} \mathrm{Pd}_{2} \mathrm{Al}_{3}$ in the region where AFM order and $\mathrm{SC}$ are observed $(0 \leq x \lesssim 0.2)$ [68]. As $x$ in the $\mathrm{U}_{1-x} \mathrm{Th}_{x} \mathrm{Pd}_{2} \mathrm{Al}_{3}$ system is increased further, a crossover occurs in the range $0.2 \lesssim x \lesssim 0.4$ to an NFL regime for $0.6 \lesssim x<1$. In the NFL regime, $\rho(T)$ and $\chi(T)$ exhibit Kondo-like behavior at high temperatures and NFL behavior at low temperatures $T<T_{K}[2,69,70]$. The NFL characteristics in $\rho(T)$, $C(T)$, and $\chi(T)$ scale with $U$ concentration $(1-x)$ and $T_{K}$, indicating that the NFL behavior is a single ion phenomenon, presumably associated with an unconventional magnetic moment screening mechanism (Kondo effect). It is not known whether the magnetic moments of the $\mathrm{U}$ ions are completely, under, or over screened by the conduction electron spins. However, there is no evidence for magnetic order down to $20 \mathrm{mK}$ from the $\rho(T)$ measurements. The U contributions to $\rho(T)$, $C(T)$, and $\chi(T)$ in $\mathrm{U}_{1-x} \mathrm{Th}_{x} \mathrm{Pd}_{2} \mathrm{Al}_{3}$ have the following $T$-dependences: (i) $\Delta \rho(T)=$ $\Delta \rho_{c}+\Delta \rho_{K}(T)$, where $\Delta \rho_{K}(T)=\Delta \rho_{K}(0)\left[1-a\left(T / T_{K}\right)^{n}\right]$ for $20 \mathrm{mK} \lesssim T \lesssim$ $20 \mathrm{~K}$, with $n \approx 1.5, T_{K} \approx 20 \mathrm{~K}$, and $a \approx 0.3$ [70]. The potential scattering term $\Delta \rho_{c}$ and the Kondo scattering contribution $\Delta \rho_{K}(0)$ both scale with U concentration $(1-x)$ and the Kondo temperature $T_{K}$ is independent of $x$, consistent with singleion behavior. (ii) $\Delta C(T) / T \propto-\left[(1-x) / T_{K}\right] \ln \left(T / T_{K}\right)[29,33]$ or $\Delta C(T) / T \propto$ $(1-x)\left(T / T_{K}\right)^{-1+\lambda}$ with $\lambda=0.8$ [35] (Fig. 6). (iii) $\Delta \chi(T) \propto(1-x)\left(T / T_{K}\right)^{-1+\lambda}$ with $\lambda \approx 0.5-0.6[2,35]$. Similar results for $\Delta \chi(T)$ were obtained by Strydom et al. [71].

Shown in Fig. 5(b) is the most recent version of the $T-x$ phase diagram of the $\mathrm{U}_{1-x} \mathrm{Y}_{x} \mathrm{Pd}_{2} \mathrm{Al}_{3}$ system [72, 73]. In contrast to the $\mathrm{U}_{1-x} \mathrm{Th}_{x} \mathrm{Pd}_{2} \mathrm{Al}_{3}$ system, $T_{N}$ and $T_{c}$ decrease rapidly with increasing $x$ in the $\mathrm{U}_{1-x} \mathrm{Y}_{x} \mathrm{Pd}_{2} \mathrm{Al}_{3}$ system. The AFM order that occurs in the region $0<x \lesssim 0.3$ is replaced by spin glass freezing in the region $0.3<x \leq x_{c} \approx 0.7$. At $x_{c}$, the spin glass freezing temperature $T_{S G}$ vanishes (QCP) and NFL behavior occurs for $0.7 \leq x \lesssim 0.85$ [72].

Displayed in Fig. 7 are $\rho$ vs. $T, C / T$ vs. $\log T$ (upper inset), and $\chi$ vs. $T^{1 / 2}$ (lower inset) data for a $\mathrm{U}_{1-x} \mathrm{Y}_{x} \mathrm{Pd}_{2} \mathrm{Al}_{3}$ sample with a composition $(x=0.8)$ close to that of the QCP at $x_{c} \approx 0.7$. These results suggest that the NFL behavior in this system is a cooperative phenomenon arising from fluctuations of a magnetic order parameter above the spin glass freezing transition at $T=0 \mathrm{~K}$. In the $\mathrm{U}_{1-x} \mathrm{Y}_{x} \mathrm{Pd}_{2} \mathrm{Al}_{3}$ system, $\rho(T), C(T)$, and $\chi(T)$ have the following $T$-dependences at low temperatures [72, 73]. (i) $\rho$ (T) $\propto T^{n}(0.1 \mathrm{~K} \lesssim T \lesssim 7 \mathrm{~K})$ where $n$ evolves from 1.5 for samples in the vicinity of the QCP to 1 for $x>0.8$. (ii) $C(T) / T \propto-\ln T$ or $T^{-1+\lambda}$ with $\lambda \approx 0.8$ $(0.6 \lesssim T \lesssim 5 \mathrm{~K})$. (iii) $\chi(T) \propto\left[1-\left(T / T_{0}\right)^{1 / 2}\right]$ with $T_{0} \approx 30 \mathrm{~K}(0.4 \mathrm{~K} \lesssim \mathrm{T} \lesssim 7 \mathrm{~K})$. It is interesting that $\rho$ is predicted to vary as $T^{1.5}$ for $3 \mathrm{D}$ and $T$ for $2 \mathrm{D}$ near an AFM 
Fig. 5

Temperature-composition $(T-x)$ phase diagrams for

(a) $\mathrm{U}_{1-x} \mathrm{Th}_{x} \mathrm{Pd}_{2} \mathrm{Al}_{3}$,

(b) $\mathrm{U}_{1-x} \mathrm{Y}_{x} \mathrm{Pd}_{2} \mathrm{Al}_{3}$, and

(c) $\mathrm{U}_{1-x} \mathrm{La}_{x} \mathrm{Pd}_{2} \mathrm{Al}_{3}$.

Antiferromagnetic (AFM), spin

glass (SG), superconducting

(SC), and non-Fermi liquid

(NFL) regions are identified in the figures. After Ref. [74]

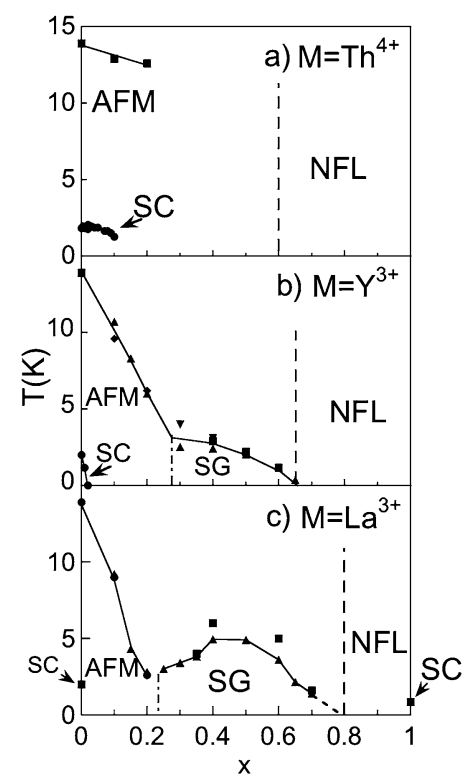

Fig. 6 Specific heat $C$ divided by temperature $T$ for $\mathrm{U}_{1-x} \mathrm{Th}_{x} \mathrm{Pd}_{2} \mathrm{Al}_{3}$ for $x=0.4$, 0.6 , and 0.8 . For all of these concentrations, there is a low temperature upturn in $C / T$ that is indicative of non-Fermi-liquid (NFL) behavior

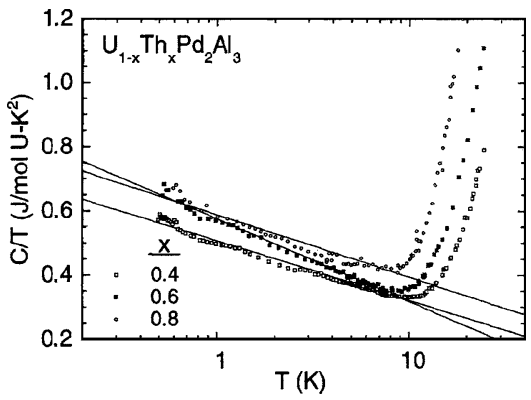

QCP. However, there is no evidence for a dimensional crossover in this system near $x=0.8$.

The $T-x$ phase diagram of the $\mathrm{U}_{1-x} \mathrm{La}_{x} \mathrm{Pd}_{2} \mathrm{Al}_{3}$ system shown in Fig. 5(c) is very similar to that of the Y-substituted system; both show the suppression of $T_{N}$, giving way to spin glass freezing at higher substituent concentrations, followed by NFL behavior where $T_{S G}$ is suppressed to zero [76]. The fact that substituting $\mathrm{UPd}_{2} \mathrm{Al}_{3}$ with trivalent $\mathrm{La}$ and $\mathrm{Y}$ results in similar phase diagrams, in contrast to tetravalent thorium, supports the idea that the valence of the substituent atom is primarily responsible for the magnetic properties of the system. For the $\mathrm{U}_{1-x} \mathrm{Y}_{x} \mathrm{Pd}_{2} \mathrm{Al}_{3}$ system with $x=0.8$ and 0.9 , the specific heat $C(T) / T \propto-\ln T$, similar to the La and Th substituted systems. The resistivity can be fit by the expression $\rho(T) / \rho_{0}=1-a T^{n}$ where $n \sim 1.3$.

Shown in Fig. 8 is the resistivity $\rho(T)$ for a sample with $x=0.8$ in the NFL regime for each of the three systems $\mathrm{U}_{1-x} M_{x} \mathrm{Pd}_{2} \mathrm{Al}_{3}(M=\mathrm{Th}, \mathrm{Y}, \mathrm{La})$ [74]. At low temperatures, $\rho(T)$ behaves as a power law with $n \sim 1.5$ for all three systems; however, $\rho(T)$ increases with $T$ for the Y substituted system, and decreases with $T$ for the La and 
Fig. 7 Electrical resistivity $\rho$ vs. $T$, specific heat divided by temperature, $C / T$, vs. $\ln T$ (upper inset), and magnetic susceptibility $\chi$ vs. $T^{1 / 2}$ (lower inset) for $\mathrm{U}_{0.2} \mathrm{Y}_{0.8} \mathrm{Pd}_{2} \mathrm{Al}_{3}$. After Ref. [75]

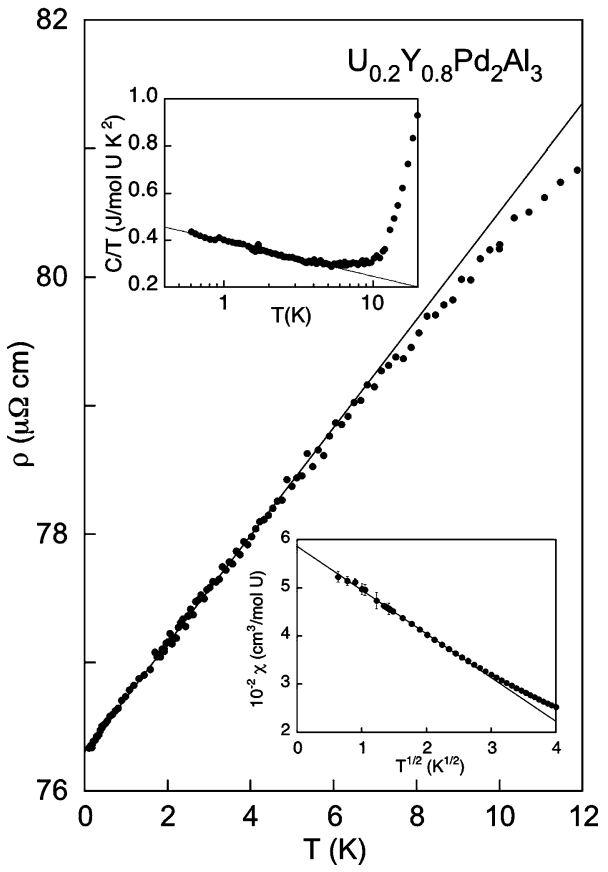

Fig. 8 Electrical resistivity $\rho$ normalized to its value at $300 \mathrm{~K}$ as a function of temperature $T$ for samples of $\mathrm{U}_{0.2} M_{0.8} \mathrm{Pd}_{2} \mathrm{Al}_{3}$ ( $M=\mathrm{Th}, \mathrm{Y}, \mathrm{La})$. The inset shows the unit cell volume $V$ determined from powder X-ray diffraction as a function of $x$ for the $\mathrm{U}_{1-x} M_{x} \mathrm{Pd}_{2} \mathrm{Al}_{3}$ systems. After Ref. [74]

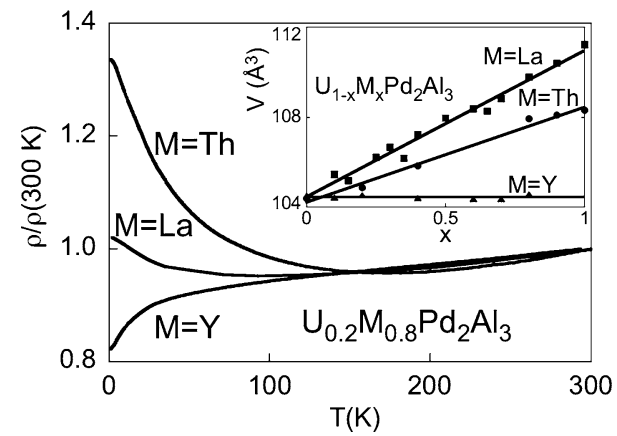

Th substituted systems (see Fig. 8). These different behaviors of $\rho(T)$ correlate with the lattice parameters in these systems [76], which are shown in the inset to Fig. 8. Substitution of La or Th results in an expansion of the hexagonal lattice of $\mathrm{UPd}_{2} \mathrm{Al}_{3}$ and an increase in $\rho(T)$ with decreasing $T$, whereas substitution of Y leaves the lattice virtually unchanged and a decrease of $\rho(T)$ with decreasing $T$. However, the correlations between the change in $\rho(T)$ and the lattice parameter is not quantitative; the change in $\rho(T)$ is larger for Th substitution, while the change in the lattice parameter is larger for La substitution. The experiments on the $\mathrm{U}_{1-x} M_{x} \mathrm{Pd}_{2} \mathrm{Al}_{3}(M=\mathrm{Th}$, $\mathrm{Y}, \mathrm{La}$ ) systems reveal that there are two possible routes to NFL behavior, a singleion mechanism associated with an unconventional Kondo effect, and an inter-ionic interaction mechanism involving order parameter fluctuations near a second-order phase transition that has been suppressed to $0 \mathrm{~K}$. Although atomic disorder is obvi- 
ously present in these pseudoternary systems, it does not appear to be the dominant underlying mechanism for the NFL behavior, in view of the similarity of the NFL characteristics for chemically substituted and stoichiometric $f$-electron materials.

\section{$4 \mathrm{URu}_{2} \mathrm{Si}_{2}$}

The heavy fermion compound $\mathrm{URu}_{2} \mathrm{Si}_{2}$ undergoes two ordering transitions: below about $1.5 \mathrm{~K}$ it is a superconductor, while at higher temperatures but below $17.5 \mathrm{~K}$, it is in a hidden order (HO) phase [77-79]. The latter ordered phase has been the subject of much attention and disagreement over the more than two decades since the discovery of the compound. The HO phase is so named because its ordering transition is accompanied by a large BCS-like specific heat anomaly [77] whose entropy cannot be accounted for by the small associated antiferromagnetic moment [80]. While it is now widely accepted that the antiferromagnetic moment is not intrinsic to the HO phase [81], the search for an alternative order parameter continues. Theoretical descriptions of the $\mathrm{HO}$ parameter can be broadly divided into those based on local or itinerant degrees of freedom, as indicated by some recent proposals, which include quadrupolar order [82], nesting-driven [83, 84], and unconventional Kondo scenarios [85]. Although the microscopic order parameter has not yet been definitively established, a multitude of bulk property measurements indicate that a partial gapping of the Fermi surface is involved in the HO transition. In addition to the BCS-like specific heat anomaly, there are anomalies in the electrical resistivity, magnetic susceptibility [77-79], ultrasound [86], thermal expansion [87], and an increase in lattice thermal conductivity [88]. Additional evidence points to the opening of a large gap in the tunneling spectrum [89], the incommensurate spin excitation spectrum [90], and the crossing of the chemical potential by a heavy electron band [91].

Identifying NLF behavior in $\mathrm{URu}_{2} \mathrm{Si}_{2}$ is complicated by the rather complex $T$ dependence of its bulk properties under ambient conditions. Both the magnetic susceptibility and electrical resistivity are anisotropic [78, 79], and show characteristics typical of Kondo lattice materials. The magnetic susceptibility $\chi(T)$ follows a Curie-Weiss law at high temperatures, reflecting a uranium free ion moment, but deviates from this behavior above $100 \mathrm{~K}$ and peaks at $50 \mathrm{~K}$. At low temperatures, $\chi(T)$ saturates to a value of $\chi_{0} \approx 0.005 \mathrm{~cm}^{3} \mathrm{~mol}^{-1}$. The electrical resistivity $\rho(T)$ has a negative slope between room temperature and $75 \mathrm{~K}$, where it reaches a maximum and then decreases dramatically, which may be attributed to Kondo coherence. Well below the HO transition temperature, $\rho(T)$ has been described both by $T^{2}$ Fermi liquid behavior [92-94], and $T^{n}$ dependence, where $n<2$ [95, 96]. Given the presence of the two phase transitions, the electronic specific heat $C(T)$ is also somewhat complicated. It is possible to estimate $T$-linear Fermi liquid-like $\gamma_{0}$ terms both above $\left(\sim 150 \mathrm{~mJ} \mathrm{~mol}^{-1} \mathrm{~K}^{-2}\right)$ and below $\left(\sim 60 \mathrm{~mJ} \mathrm{~mol}^{-1} \mathrm{~K}^{-2}\right)$ the transition $[77$, $79,90]$, and the Sommerfeld-Wilson ratio $\chi_{0} / \gamma_{0}$ is on the order of one. However, below $10 \mathrm{~K}$ but above the superconducting anomaly, $C / T$ actually has a negative slope, well-described by logarithmic or weak power-law $T$-dependence [77]. Taken together, these properties are broadly consistent with Fermi liquid behavior, although 
the agreement may be fortuitous because the $\mathrm{HO}$ state of $\mathrm{URu}_{2} \mathrm{Si}_{2}$ is not a paramagnetic metal, and the true low temperature limiting behavior is masked by the superconducting phase.

In order to find signatures of NFL behavior in $\mathrm{URu}_{2} \mathrm{Si}_{2}$, it is thus necessary to tune the system away from its superconducting ground state. Such tuning is generally accomplished in the laboratory by the application of external pressure, magnetic field, or chemical substitution, and all three approaches are well represented in the literature. While the application of pressure does suppress superconductivity [92], it also transitions the system from $\mathrm{HO}$ into an antiferromagnetic state at $8 \mathrm{kbar}[81,96]$. Despite this change in ground state, the anomalies in the bulk properties associated with the $\mathrm{HO}$ and antiferromagnetic phase transitions are similar and changes in the $T$ dependence are subtle between the two phases [93, 95, 97, 98]. For example, it is possible to fit $\rho(T)$ with a $T^{2}$ term to at least $20 \mathrm{kbar}[95,97]$. Complementary low$T$ magnetic and calorimetric data are lacking. The absolute value of $\chi(T)$ is difficult to measure under pressure because of the small moment [99]. However, early measurements of $C(T)$ under pressure seem to suggest that the magnitude of the negative slope in $C / T$ is diminished by pressure [100]. Consequently, there is little evidence for the presence of NFL behavior under pressure.

A number of high magnetic field studies on $\mathrm{URu}_{2} \mathrm{Si}_{2}$ have uncovered a rich phase diagram in the vicinity of the critical field of the $\mathrm{HO}$ phase. Building on early measurements that showed multiple high field transitions [101, 102], it has been determined that there are at least five ordered phases between $30 \mathrm{~T}$ and $40 \mathrm{~T}$ [103-106]. Much of the novel physics appears related to a Fermi surface reconstruction near $38 \mathrm{~T}$ associated with polarization of the heavy electrons [104, 105, 107]. Magnetization and electrical resistivity studies suggest that a high-field quantum critical point is masked or "protected" by an ordered phase [104, 105]. The $T$ dependence of the resistivity outside of this phase, for $T>3 \mathrm{~K}$, can be described by power laws $\rho(T) \sim T^{n}$ with $n<1$ [105]. This effectively defines a fan-like NFL region in the $H-T$ plane emanating from the $H$-tuned quantum critical point hidden under the ordered phase. At lower $T, \rho(T)$ fits exhibit $n<2$ in several of the ordered phases for fields below 39 T. At higher fields, in the field-polarized paramagnetic phase, $\rho(T)$ exhibits a $T^{2}$ dependence, with an $A$ coefficient that diverges as the critical field is approached from high field. Surprisingly, these high $H$ features persist even in Rh-substituted samples, in which the HO is suppressed, indicating that the quantum critical behavior is a phenomenon independent of the HO [108, 109]. Non-Fermi liquid behavior has also been identified at lower fields, just above the superconducting critical field, although still well into the HO phase, where the in-plane $\rho(T)$ is linear in $T$ to millikelvin temperatures [110]. High field calorimetric or magnetic evidence for NFL behavior has not been reported.

Chemical substitution, particularly on the $\mathrm{Ru}$ site $\left(\mathrm{URu}_{2-x} M_{x} \mathrm{Si}_{2}\right.$ for transition metal $M$ ), also has yielded very rich physics [111,112]. The HO phase is typically suppressed by about $x=0.1(5 \%)$, while at higher substituent concentration, long range magnetism emerges. The most extensively studied examples have been antiferromagnetism in the case of Rh substitution [113] and ferromagnetism in the case of Re substitution [114]. Details of the phase diagrams of these two systems were recently compared [115]. In fact $\mathrm{URu}_{2-x} \mathrm{Re}_{x} \mathrm{Si}_{2}$, studied extensively in our laboratory, is the first known example of a ferromagnetic heavy fermion compound, with a 
Fig. 9 Strength of the logarithmic low $T$ divergence in $C / T$ in polycrystalline $\mathrm{URu}_{2-x} \mathrm{Re}_{x} \mathrm{Si}_{2}$. The inset shows how the divergence is strongest near $x=0.3$

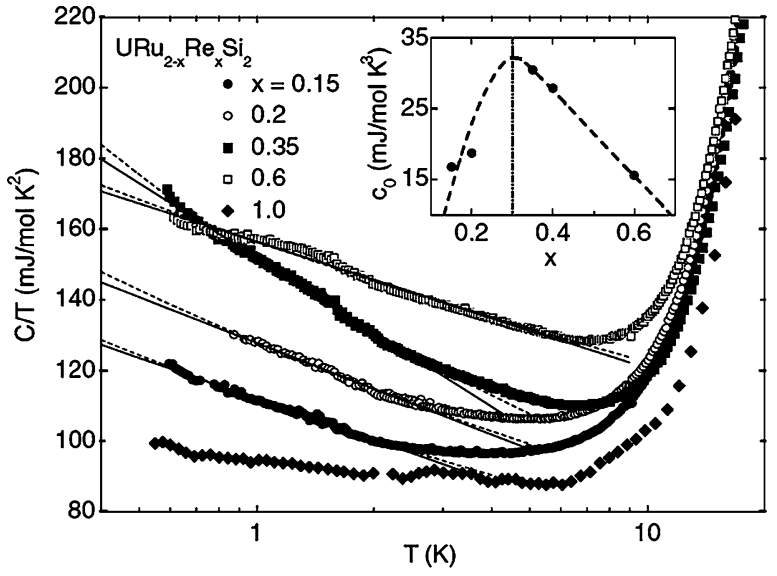

maximum Curie temperature of $40 \mathrm{~K}$ at $x=0.8$, which is very near the Re solubility limit at $x=1$. The ferromagnetic state, which features only a small moment, has been confirmed by neutron scattering and ${ }^{29} \mathrm{Si}$ NMR $[116,117]$. The magnetism appears to have an itinerant origin: in addition to the small moment, there are no anomalies in electrical resistivity or specific heat at the Curie temperature. The heavy fermion state gives rise to an enhanced electronic specific heat [111] and a narrow Drude peak in optical conductivity [118].

Most interestingly, archetypal NFL behavior has been identified in the bulk properties of $\mathrm{URu}_{2-x} \mathrm{Re}_{x} \mathrm{Si}_{2}$ : power law $T$ dependence in $\rho(T)$ and $\chi(T)$, and weak power law or logarithmic divergence in $C(T) / T$ at low $T$, shown in Fig. 9 [119]. However, these NFL phenomena are rather unique, as they are found inside the ferromagnetic phase, in sharp contrast to the "conventional" occurrence of NFL behavior in the unordered or paramagnetic state. The behavior was tentatively ascribed to a quantum Griffiths phase scenario, which could in principle explain the power-law $T$-dependence and is consistent with the expected important role of substitutional disorder in this system. The case for NFL behavior was strengthened by a subsequent inelastic neutron scattering study, which found energy-temperature $(\omega / T)$ scaling in the dynamic magnetic susceptibility [120]. The significance of this behavior, identified in varied NFL materials like $\mathrm{UCu}_{5-x} \mathrm{Pd}_{x}$ [62] and $\mathrm{Sc}_{1-x} \mathrm{U}_{x} \mathrm{Pd}_{3}$ [61], is that the temperature itself acts like the energy scale in the system, as would happen in the absence of an effective Fermi energy. Also, the scaling exponents determined from $\omega / T$ scaling agree with the NFL exponents from the power-law $T$ dependence in bulk $\chi(T)$.

A major source of uncertainty in the preceding studies on $\mathrm{URu}_{2-x} \mathrm{Re}_{x} \mathrm{Si}_{2}$ was the polycrystalline microstructure of the samples, which could lead to disorder or directional averaging. Nonetheless, the physical properties of single crystals of $\mathrm{URu}_{2-x} \mathrm{Re}_{x} \mathrm{Si}_{2}$ do generally agree with the earlier bulk measurements, although some new and interesting information has been revealed [121]. A phase diagram based on the measurements on single crystals is shown in Fig. 10. Because the bulk magnetism in $\mathrm{URu}_{2-x} \mathrm{Re}_{x} \mathrm{Si}_{2}$ is very Ising-like, with an easy magnetic $c$-axis, oriented single crystals allowed a comprehensive study of the finite-temperature (classical) critical scaling as the ferromagnetic quantum phase transition is approached 
Fig. 10 (Color online) Phase diagram of $\mathrm{URu}_{2-x} \mathrm{Re}_{x} \mathrm{Si}_{2}$ derived from measurements on single crystals. The upper panel shows the evolution of two parameters associated with NFL behavior: the $\rho(T)$ power law exponent, which is always less than 2, and the coefficient of the divergent logarithmic term in $C / T$. The lower panel shows the suppression of the hidden order phase and the onset of ferromagnetism with increased Re concentration. The ferromagnetic phase boundary is derived from an unconventional scaling analysis [65]
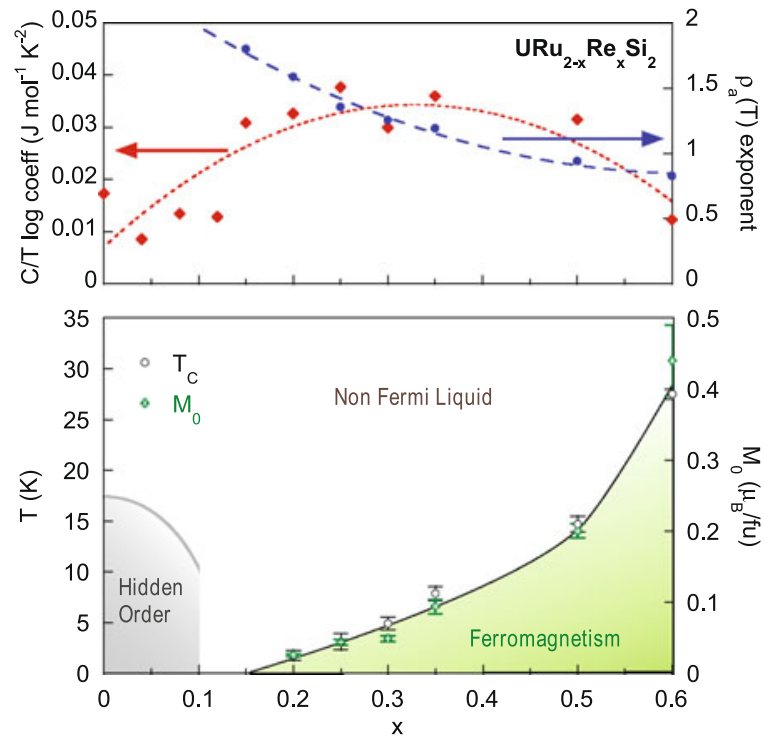

from high $x$ [65]. The results are unprecedented: the magnetic exponents are not constant as the Curie temperature varies. Instead, $\delta$ and $\gamma$ are suppressed continuously towards the critical concentration, implying a continual change in symmetry class, a rather unconventional scenario. Despite this, the exponents $\gamma$ match the NFL-like $\chi(T)$ exponents determined previously [119], providing a consistent explanation. In addition, the ferromagnetic critical concentration has been identified to be less than $x=0.2$, which is quite close to the extrapolated endpoint of the HO phase boundary near $x=0.15$. This raises the exciting possibility that the two phases share a common critical endpoint, which may lie at the heart of the unusual physics in this system. Future neutron scattering measurements on single crystals will provide important insight into this open question.

\section{$5 \mathrm{CeIn}_{3}$ and $\mathrm{CeMIn} 5(M=\mathrm{Co}, \mathrm{Ir}, \mathrm{Rh})$}

The family of compounds $\mathrm{Ce}_{n} T_{m} \operatorname{In}_{3 n+2 m}$, with $T=\mathrm{Co}$, Rh and Ir, $n=1,2$ and $m=0,1$ [122], has continuously attracted scientific interest in the context of heavy fermion superconductivity since its discovery in the late 1990ies. This class of materials displays a variety of exciting phenomena including superconductivity (with and without application of pressure), antiferromagnetism, valence fluctuations, Kondo physics and NFL behavior.

The cubic parent compound $\mathrm{CeIn}_{3}$ orders antiferromagnetically below a Néel temperature $T_{N} \approx 10 \mathrm{~K}$ that can be suppressed by application of pressure and vanishes at $P_{N} \approx 25 \mathrm{kbar}$ [123]. The pressure phase diagram of $\mathrm{CeIn}_{3}$ is prototypical for an antiferromagnetic QCP scenario (Hertz-Millis theory [40, 41]): the temperature dependence of the electrical resistivity changes from a nearly quadratic temperature dependence at ambient pressure to $\Delta \rho \sim T^{1.5}$ in a narrow interval around $P_{N}$ and back 
to ideal $T^{2}$-Fermi-liquid behavior in the paramagnetic phase [124, 125]. De Haas-van Alphen measurements confirm that the electronic state of the $4 f$-electrons changes from local to itinerant character at $P_{N}$ [126], demonstrating that the magnetic phase diagram is driven by the competition between long-range RKKY and Kondo interactions as in the classical Doniach picture [127]. On the high pressure side of $P_{N}$, the Kondo interactions, in turn, lead to the formation of a heavy Fermi-liquid. However, the existence of a QCP is contradicted by recent NQR measurements that suggest that the QPT at $P_{N}$ is first order [128]. In a narrow dome around $P_{N}$ and below $T_{c}=0.19 \mathrm{~K}\left(\right.$ at $\left.P_{N}\right)$, superconductivity is observed for high purity samples (residual resistivity $<1 \mu \Omega \mathrm{cm}$ ) [124]. The coexistence of AFM order and superconductivity around a putative QCP demonstrates that the superconductivity is unconventional in nature. The strong magnetic interactions between the $4 f$ magnetic moments and the itinerant electrons suggests that the superconductivity may be mediated by magnetic interactions rather by phonons, as for conventional superconductors [18].

The series $\mathrm{Ce} \mathrm{In}_{5}$ with $T=\mathrm{Co}, \mathrm{Rh}$, Ir is crystallographically related to the parent compound $\mathrm{CeIn}_{3}$. The tetragonal structure of $\mathrm{Ce} T \mathrm{In}_{5}$ can be viewed as a layered structure where building blocks consisting of $\mathrm{CeIn}_{3}$ and $T \mathrm{In}_{2}$ are stacked, one upon the other, in an alternating fashion. At ambient pressure, $\mathrm{CeRhIn}{ }_{5}$ exhibits antiferromagnetic order with a propagation vector $\mathbf{k}=(0.5,0.5,0.297)$ at $T_{N}=3.8 \mathrm{~K}$ [129], while CeIrIn 5 [130] and $\mathrm{CeCoIn}_{5}$ [131] both evince superconductivity below $T_{c}=0.4 \mathrm{~K}$ and $T_{c}=2.3 \mathrm{~K}$, respectively. Here the latter is the highest $T_{c}$ of the known Ce-based heavy-fermion superconductors [131]. In CeRhIn ${ }_{5}$, superconductivity develops out of a NFL normal state above an applied pressure of 16.3 kbar [19]. For increasing pressure, $T_{c}$ is reported to increase towards approximately $2 \mathrm{~K}$ at about $30 \mathrm{kbar}$, followed by a decrease and the eventual suppression of superconductivity at $85 \mathrm{kbar}$ [132]. More recent measurement of the electrical resistivity, specific heat and magnetic susceptibility at low temperature, however, suggest that superconductivity in CeRhIn ${ }_{5}$ develops below $T_{c}=110 \mathrm{mK}$ within the AF phase [133]. It is interesting to note that the $T_{c}$ increases by an order of magnitude from the $3 \mathrm{D}$ cubic $\mathrm{CeIn}_{3}$ towards the quasi-2D tetragonal compound $\mathrm{CeCoIn}_{5}$ with the largest lattice anisotropy $c / a$. This is in agreement with mean-field theory of superconductivity for nearly antiferromagnetic metals which demonstrates that magnetically mediated superconductivity is more stable in quasi-2D compared to 3D [134]. Monthoux et al. [134], however, emphasize that this kind of magnetically mediated superconductivity is sensitive to small changes in the band structure, possibly yielding an explanation why CeRhIn 5 with a $c / a$ ratio intermediate between $\mathrm{CeIrIn}_{5}$ and $\mathrm{CeCoIn}_{5}$ only exhibits superconductivity below $T_{c}=110 \mathrm{mK}$. For the entire series of Ce $T \mathrm{In}_{5}$ compounds, both the specific heat [135] and the spin-lattice relaxation rate divided by temperature $1 / T_{1} T$, measured by means of NQR [136-138], provide compelling evidence for the existence of line nodes in the superconducting energy gap and therefore unconventional superconductivity.

In particular, $\mathrm{CeCoIn}_{5}$ continues to generate great interest, as it exhibits strong Pauli paramagnetic effects [139] that, combined with its quasi-2D structure and super-clean crystal properties [140, 141], fulfill the stringent requirements for the stabilization of the inhomogeneous Fulde-Ferrell-Larkin-Ovchinnikov (FFLO) state[142, 143]. However, up to now, unambiguous microscopic proof for the existence of the FFLO state does not exist, in spite of the multitude of experimental 
signatures that were reported that suggest the formation of a FFLO phase [144-148]. The situation is additionally complicated by the existence of a magnetic field-induced long-range AFM phase (Q-phase) that was recently observed by means of NQR [149] and neutron scattering [150] experiments. This magnetic state emerges in the superconducting mixed state and is observed in the same high-field and low temperature region of the phase diagram that was proposed for the FFLO state. The ordering wave vector if the AFM order is observed to be field independent in contradiction with the predictions for the FFLO state. This rules out the existence of the FFLO state in $\mathrm{CeCoIn}_{5}$ and new theoretical work on this issue suggests that the Q-phase represents a new exotic state at high fields in which a pattern of coexisting SCing condensates develops [151].

All three compounds show pronounced NFL features in their physical properties above $T_{c}$ or when the superconducting phase is suppressed by either temperature, pressure, chemical substitution, or magnetic field. An overview of some of the corresponding NFL exponents is provided in Table 4 of Ref. [152]. In CeIrIn 5 , the electrical resistivity increases as $\rho(T)-\rho\left(T_{0}\right) \propto T^{n}$ with $n=1.3 \pm 0.05$ for $T_{0} \leq T \leq 5 \mathrm{~K}$ [130]. Here $T_{0}$ is the temperature where the resistivity drops below the instrumental resolution above the actual bulk superconducting transition observed in the specific heat and the magnetic susceptibility. When $T_{0}$ is suppressed by application of a magnetic field the NFL resistivity can be observed down to $60 \mathrm{mK}$. Specific heat measurements in zero field show that $C / T$ can be fitted as $\gamma-A T$ for $0.4 \mathrm{~K}<T<2.5 \mathrm{~K}$ [153]. In a magnetic field of $6 \mathrm{~T}$ applied perpendicular to the basal plane, this changes to $\gamma_{0}-A T^{1 / 2}$ for $0.6<T<6 \mathrm{~K}$ which compares well to NFL theory for 3D antiferromagnetic fluctuations [38, 41]. In CeCoIn 5 , the resistivity $\rho(T)-\rho_{0}$ was found to be linear in $T$ above the superconducting transition and up to pressures of 1.6 GPa [154]. Such behavior is expected for 2D antiferromagnetic quantum-critical system [44] and leads to the proposal that $\mathrm{CeCoIn}_{5}$ is near an AFM QCP situated at slightly negative pressures [154]. At higher pressure, a FL-like resistivity $\rho(T)-\rho_{0} \sim T^{2}$ is recovered between $T_{c}$ and a cross-over temperature $T_{F L}\left(\lesssim 2.5 \mathrm{~K}\right.$ ), whereas above $T_{F L}$ and up to $60 \mathrm{~K}, \rho(T)-\rho_{0} \sim T^{1.5}$ is observed. The low temperature specific heat can be best fit by $C / T \sim-\ln T$ which is also consistent with NFL behavior. $\mathrm{CeCoIn}_{5}$ is particularly interesting because magnetoresistance and specific heat measurements suggest the existence of a QCP situated at the upper critical field $H_{c 2}(T)=4.95 \mathrm{~T}[155,156]$. Regardless of the field direction, the normal state properties retain their NFL character up to $H_{c 2}$; above the upper critical field and below a cross-over temperature, FL behavior of the resistivity is finally restored. In CeRhIn 5 , the resistivity does not exhibit FL behavior over the entire pressure range investigated. At $P=3.2 \mathrm{kbar}$, which is where the maximum in the superconducting transitions is observed, the resistivity has linear $T$-dependence in the normal state, similar to $\mathrm{CeCoIn}_{5}$ at ambient pressure [132].

The rich physics in the series $\mathrm{Ce}_{n} \mathrm{~T}_{m} \mathrm{In}_{3 n+2 m}$ described above provides a large phase space for probing the interplay between superconductivity, magnetism, quantum criticality, Kondo and NFL phenomena. This is particularly appealing, because relatively large single crystals can be grown by means of the indium self-flux method and doping studies on all three different atomic sites are possible in this family (see e.g., [157] for a recent review). We have carried out detailed doping studies on both the $\mathrm{Ce}$ and $T$ sites. 
By substituting Co onto the Rh site in $\mathrm{CeRhIn}_{5}$, we demonstrated the $\mathrm{CeCoIn}_{5}$ is indeed near to an antiferromagnetic QCP $[158,159]$. We tracked both $T_{N}$ and $T_{c}$ in the electrical resistivity, specific heat and magnetic susceptibility over the entire doping range and established that the AFM state in $\mathrm{CeRh}_{1-x} \mathrm{Co}_{x} \mathrm{In}_{5}$ persists up to a critical concentration $x_{c} \approx 0.75$ where $T_{N}$ is suppressed to zero. Superconductivity is observed for concentrations $0.4 \lesssim x$ below $T_{c}=1.6 \mathrm{~K}$ and coexists with the AFM phase. Up to $x \approx x_{c}$, it remains insensitive to the Co concentration where it increases up to $2.3 \mathrm{~K}$ for pure $\mathrm{CeCoIn}_{5}$. Around the putative AFM QCP both the residual resistivity and entropy are significantly increased (Fig. 11). Finally, for selected Co concentrations $(x=0.1,0.2,0.4$ and 0.6$)$, the electrical resistivity was also studied under hydrostatic pressure. For the samples with $x=0.1$ and 0.2 , superconductivity appears upon the application of pressure. Apart from the $x=0.6$ sample for which no AFM is observed, superconductivity is found to coexist with the AFM until the magnetic order is suppressed. It is, in particular, remarkable that the superconducting transition temperature $T_{c}$ is constant over a wide pressure range even far from the possible QCP, unlike the prototypical scenario for unconventional superconductivity mediated by magnetic fluctuations proposed in Ref. [18]. In a different study by Nicklas et al., where Ir, instead of Co, was substituted for Rh, a strikingly similar phase diagram was observed [160]. However, in this case, the application of pressure splits the broad superconducting phase into two

Fig. 11 The entropy and phase diagram of $\mathrm{CeRh}_{1-x} \mathrm{Co}_{x} \mathrm{In}_{5}$ as reported in Ref. [159] are shown. Note that the entropy increases around the position of the putative AFM QCP at the critical concentration $x_{c} \approx 0.75$

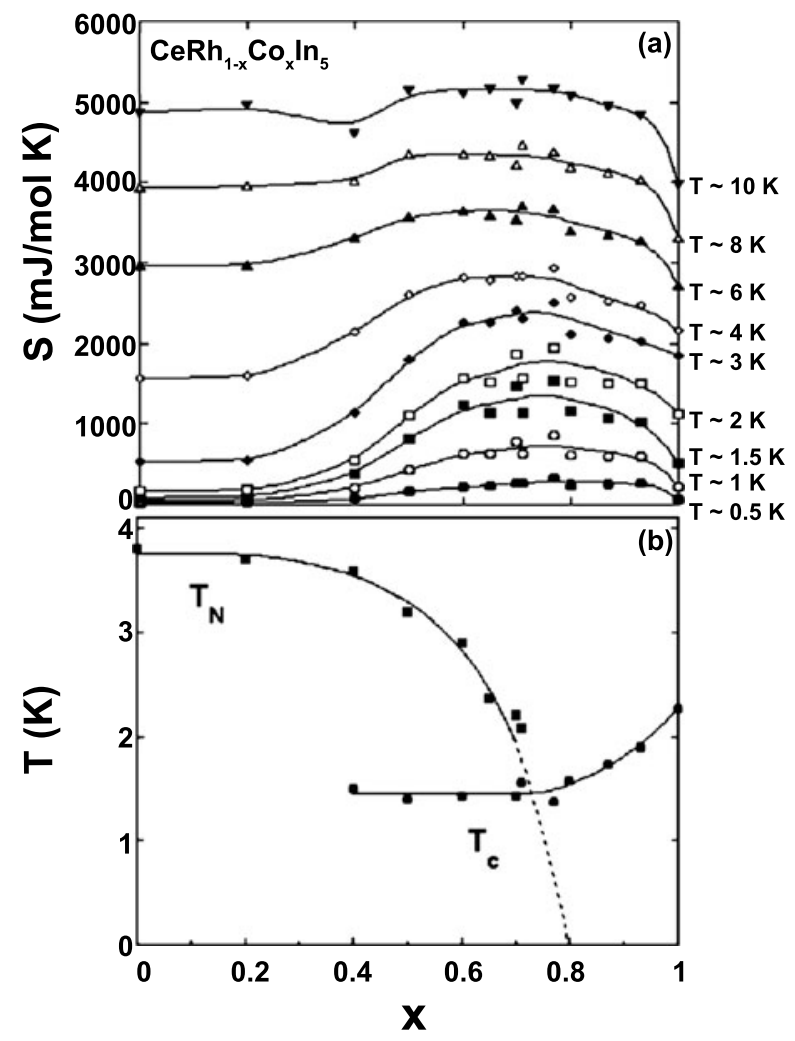


Fig. 12 The magnetic phase diagram of $\mathrm{Ce}_{1-x} \mathrm{Y}_{x} \mathrm{RhIn}_{5}$ as reported in Ref. [161] is shown. The Néel temperature $T_{N}$ vs. yttrium concentration $x$ was determined from specific heat $C(T)$, magnetic susceptibility and resistivity. The region NFL behavior is indicated for samples with $0.4 \leq x \leq 0.9$. Upper left inset: resistivity at $1.8 \mathrm{~K}$ vs. $x$. Upper right inset: magnitude of the NFL contributions to $C(T)$ (solid circles) and $\chi_{a b}(T)$ (open circles) plotted as $B / \lambda$ and $A$ where $C(T) / T=B T^{-1+\lambda}$ and $\chi_{a b}=A T^{-1+\lambda}$. Bottom inset: fraction of $\mathrm{Ce}$ ions contributing to the CEF-like effect in $C(T)$ (solid circles) and $\chi_{a b}(T)$ ('open circles)

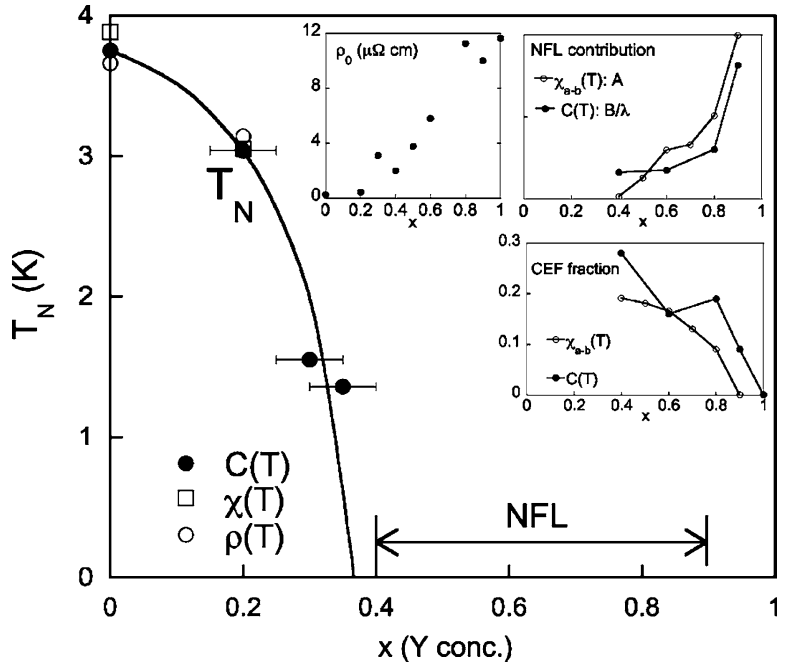

separate domes, suggesting the existence of two different pairing mechanisms. For $\mathrm{CeRh}_{1-x} \mathrm{Co}_{x} \mathrm{In}_{5}$, we also observed that the electrical resistivity in the normal state exhibits NFL behavior for all concentrations, where the exponent $n \lesssim 0.5$ on the low pressure side of the QCP associated with the AFM phase. At the critical pressure, the exponent shows a sudden jump to $n \approx 0.8$ and stays constant up to $30 \mathrm{kbar}$. We emphasize that the NFL behavior is observed over the entire investigated pressure range.

We have observed similarly persistent NFL characteristics in $\mathrm{Ce}_{1-x} \mathrm{Y}_{x} \mathrm{RhIn}_{5}$. Doping $\mathrm{Y}$ onto the Ce site suppresses the AFM order at a critical concentration $x_{c} \approx 0.38$. Above this critical concentration, specific heat and magnetic susceptibility measurements indicate NFL characteristics that can be fit with power laws reminiscent of the Griffith-McCoy singularity model after a nuclear Schottky anomaly is subtracted [161]. More interestingly, the magnitude of the respective NFL contributions is more pronounced for increasing $x$ and is observed up to $x=0.9$ (Fig. 12). The extended range of concentration and pressure over which NFL behavior is observed in both $\mathrm{CeRh}_{1-x} \mathrm{Co}_{x} \mathrm{In}_{5}$ and $\mathrm{Ce}_{1-x} \mathrm{Y}_{x} \mathrm{RhIn}_{5}$ is unusual and not in agreement with the general perception of NFL signatures arising around a QCP as observed for CeIn 3 .

In a further systematic study of the electrical resistivity for various magnetic $\left(\operatorname{Pr}^{3+}\right.$, $\left.\mathrm{Gd}^{3+}, \mathrm{Dy}^{3+}, \mathrm{Er}^{3+}\right)$ and non-magnetic $\left(\mathrm{Y}^{3+}, \mathrm{Yb}^{2+}, \mathrm{Lu}^{3+}\right)$ rare earth ion substitutions on the Ce site of $\mathrm{CeCoIn}_{5}$, we established that both the superconductivity and the Kondo-lattice are insensitive to the exact $f$-electron configuration of the substituent: both the superconducting transition temperature $T_{c}$ and the Kondo-lattice coherence temperature $T_{c o h}$ are suppressed at one universal rate as a function of residual resistivity $\rho_{0}$ for all the rare earth substitutions investigated. In stark contrast, the NFL signature in the resistivity is changed drastically from $T$-linear to sub- $T$-linear behavior by tuning the $f$-electron configuration [34]. 
Fig. 13 Pressure phase diagram of $\mathrm{MnSi}$ as reported in [29].

Here $\rho$ and $\chi$ indicate that the helical ordering temperature $T_{c}$ was determined by measurements of the electrical resistivity [171] and magnetic susceptibility [178, 179], respectively. ENS denotes elastic neutron scattering [180] that identified the cross-over temperature scale $T_{0}$ to the partial order. Larmor denotes Larmor diffraction measurements carried out to measure the lattice expansion of $\mathrm{MnSi}$ as a function of pressure and temperature (see text for details) [29]

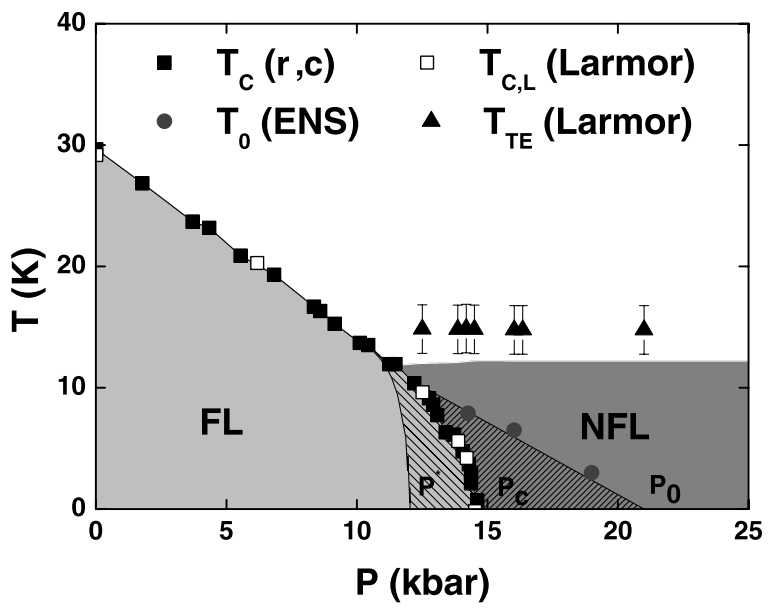

\section{$6 \mathrm{MnSi}$}

Below $T_{C}=29.5 \mathrm{~K}$, the itinerant-electron magnet $\mathrm{MnSi}$ orders in a well understood homochiral helimagnetic ground state [162-164]. The helix, with a pitch of about $180 \AA$, propagates along the space diagonals of the non-centrosymmetric cubic crystal-structure (space group $P 2{ }_{1} 3$ ). Here the lack of inversion symmetry leads to weak spin-orbit coupling, manifested in the Dzyaloshinsky-Moriya interaction (DMI) $[165,166]$, of the form $\mathbf{m} \cdot \nabla \times \mathbf{m}$. In turn, the helimagnetic ground state arises as a consequence of three competing energy scales, where the interplay between ferromagnetic exchange and the DMI stabilizes the helical spiral, that is finally weakly pinned to the $\langle 111\rangle$ direction by crystal field interactions [162].

In the helical phase, $\mathrm{MnSi}$ is well described as a weakly spin-polarized Fermi liquid [167]. The helical order can be suppressed by applying pressure and vanishes at $P_{c}=14.6$ kbar $[168,169]$ (Fig. 13). At $P_{c}$, the temperature dependence of the resistivity abruptly changes from $T^{2}$ to $T^{1.5}$, indicative of NFL behavior [169, 170]. In contrast to the commonly discussed picture of NFL behavior occurring in the vicinity of a QCP, the NFL resistivity in MnSi is observed over an extended region; arising approximately below $10 \mathrm{~K}$ near $P_{c}$, the NFL behavior has been observed down to the low mK range [171]. The NFL behavior further persists up to $45 \mathrm{kbar}$ [171-174], where the temperature exponent was reported to increase again from 1.5 [174].

In principle, the observed extended NFL resistivity makes $\mathrm{MnSi}$ a candidate for a system with a true and stable "NFL phase" far from a QPT. Interestingly, a recent calculation by Belitz et al. shows that for a helimagnet driven by the DMI, exceptionally soft Goldstone modes are expected that would lead to anomalous thermodynamic and transport properties even inside the ordered phase [175]. We have recently performed an inelastic neutron scattering study that clearly demonstrates that helimagnons indeed exist in the proposed form at ambient pressure [176].

Yet, the observed NFL behavior above $P_{c}$ has historically been discussed in terms of QPCs. The long-period helimagnetic ground state of $\mathrm{MnSi}$ is locally similar to a ferromagnet, and originally MnSi played a prominent role in the development of spin 
fluctuation theory for itinerant ferromagnetism [37, 177], in turn leading to studies searching for a "ferromagnetic" QCP $[168,169]$. However, subsequent studies established the NFL resistivity above $P_{c}$ is not driven by a QCP. AC magnetic susceptibility measurements on high purity single crystals demonstrated that the magnetic phase transition at $T_{C}$ changes from second order to first order at $P \approx 12 \mathrm{kbar}$. Additionally the existence of itinerant metamagnetism corroborates the presence of a local minimum in the free energy [178, 179].

Up to the present, critical magnetic fluctuations at $P_{c}$ that would be expected for a ferromagnetic QCP have not been identified. Interestingly, elastic neutron scattering experiments (energy resolution $50 \mu \mathrm{eV}$ ) revealed that the ordered magnetic moment does not vanish at $P_{c}$ [180]. Instead the scattering intensity of the magnetic Bragg reflections at ambient pressure is redistributed onto a small sphere in reciprocal space with a radius corresponding approximately to the pitch of the helix. This suggests that the helices have lost their long-range directional order and a "partial order" similar to liquid crystals develops. Broad maxima are observed on the sphere in the $\langle 110\rangle$ direction differing from the $\langle 111\rangle$ propagation direction of the helix below $P_{c}$. The maxima appear below a cross-over temperature $T_{0}$ that extrapolates to zero at $P_{0} \approx 21 \mathrm{kbar}$ (Fig. 13). In $\mu$ SR measurements performed by Uemera et al., no magnetic signal was observed above $P_{c}$ [181]. This suggests that the partial order below $T_{0}$ is not static on the slower timescales probed by $\mu \mathrm{SR}$, but is dynamic on time scales between $10^{-11}$ and $10^{-10} \mathrm{~s}$. In this case $T_{0}$ represents a freezing temperature below which the fluctuations of the partial order become slow enough to be observed in elastic neutron scattering or NMR [182].

Consequently, the question arises as to whether the anomalous transport properties can be explained by a new QCP situated at $P_{0}$. At a pressure controlled QCP, a change of sign is expected in the Grüneisen parameter [183]. Using this criterion, thermal expansion measurements under pressure performed by means of neutron Larmor diffraction recently established that neither the transitions at $P_{c}$ nor at $P_{0}$ can be associated with a QCP [29].

In our recent extensive inelastic neutron scattering study of the helimagnetic phase at ambient pressure, we pursued the route to NFL-like behavior proposed by Belitz et al. Their calculation shows that within the ordered helimagnetic phase anomalous soft helimagnon modes would lead to a $\Delta \rho \sim T^{5 / 2}$ resistivity for the clean limit. For a more realistic scenario, including weak disorder effects as expected near to the phase transition, the leading term in the resistivity caused by helimagnons is linear in temperature [184], in contrast to the observed $T^{1.5}$ dependence. Before our study, no experimental results about the nature of the helimagnetic Goldstone modes were available, partly because the experimental conditions are extremely challenging. High resolution in both $\mathbf{Q}$ and energy is required due to the tiny size of the magnetic Brillouin zone defined by the small helical propagation vector $k=0.035 \AA^{-1}$.

Our results reveal the existence of anomalously broad dispersive modes as illustrated in Fig. 14 [176]. A naive interpretation of the data suggests an extreme form of broadening caused by an exotic damping mechanism. However, using a model based on the theory in Ref. [175], one obtains a quantitatively precise and complete account of these helimagnon bands. For the fit of the neutron data only three parameters have been used, namely, the measured pitch of the helix, the measured spin wave stiffness 

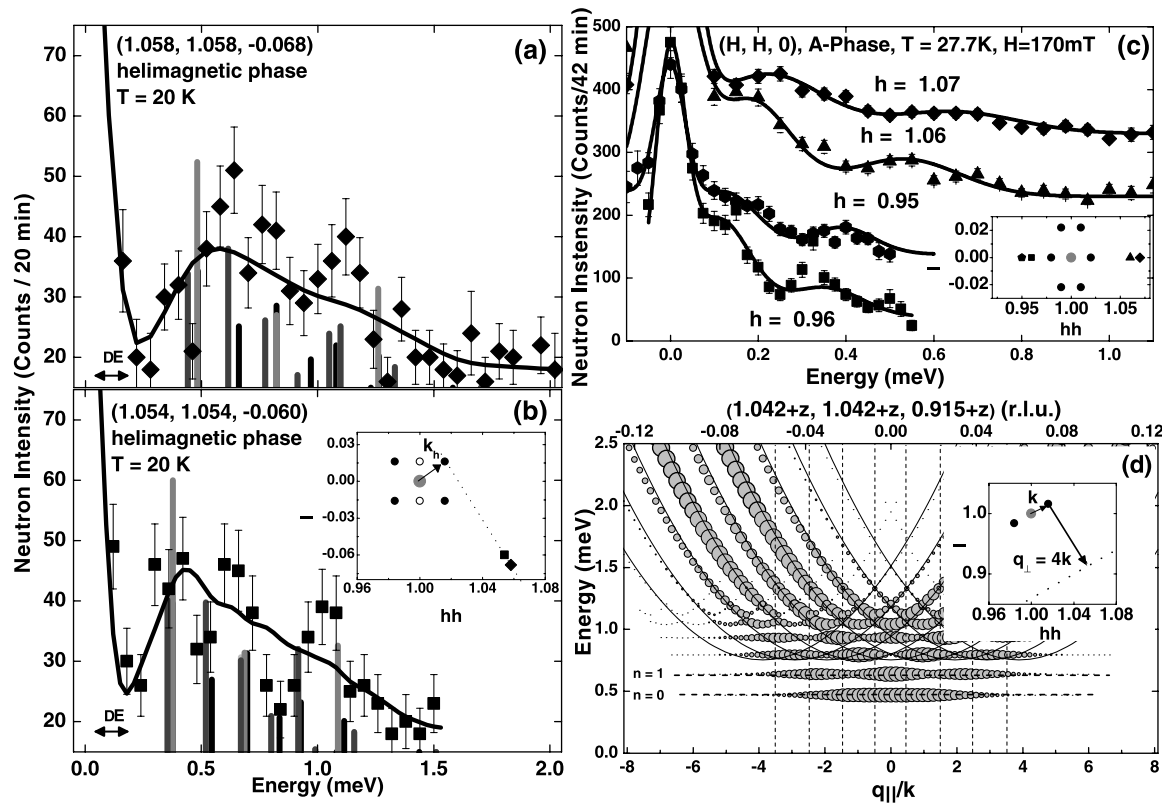

(b)

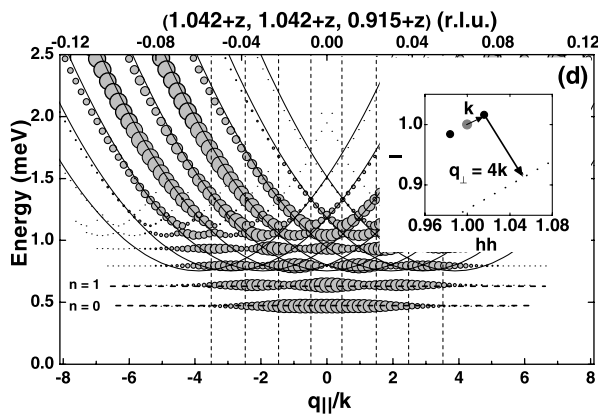

Fig. 14 Recent results highlighting the existence of helimagnons in MnSi as reported in [176, 191]. Panels (a) and (b) show typical energy scans across the magnetic excitations in the helimagnetic phase at $T=20 \mathrm{~K}$. The inset in (b) shows the position of the scans around the $(1,1,0)$ nuclear Bragg reflection (gray point) and the magnetic satellite reflections of the four magnetic $k$-domains within (black) and out of the scattering plane (empty points). The solid black line is the fit that was obtained by convolution of the instrumental resolution $\Delta E$ with the theoretical peaks that are denoted in different gray shades indicating contributions from the four domains. (c) Excitations in the A-phase at $H=170 \mathrm{mT}$ and $27.2 \mathrm{~K}$. The solid lines are multiple Gaussian fits to the data based on no particular theory. Note, however that the observed dispersive modes are very similar to the helimagnons observed in the helimagnetic phase. (d) Illustration of characteristic features of helimagnon bands. For trajectories in reciprocal space (dashed line in inset) with a finite component $q_{\perp}$ of the wave vector perpendicular to the helical propagation vector $\mathbf{k}_{h}$ (here $q_{\perp}=4 k_{h}$ ) multiple bands with approximately equal weight are excited due to Umklapp scattering at the boundary of the tiny magnetic Brillouin zone (vertical dashed lines). The strong Umklapp scattering stops the motion of spin excitations with small $q_{\|}$leading to flat bands (denoted by horizontal dashed lines). The spectral weight of the corresponding modes is proportional to the area of the points, where a maximal size cut-off was used for better visibility. For clarity only one of the existing four $k$-domains is shown

in the ferromagnetic phase (both fixed by previous experiments), and a single scale factor for the overall amplitude of the signal as the only free parameter. In particular, the abundance of helimagnon bands turns out to be a new universal property of spin excitations in chiral magnets that is driven by multiple, strong Umklapp scattering in small magnetic Brillouin zones.

Belitz et al. calculated an estimate for the helimagnon contribution to the electrical resistivity based on realistic values for $\mathrm{MnSi}$. The results demonstrate that the helimagnon contribution would be negligible compared to the Fermi-liquid contribution [184]. Therefore, the good agreement between theory and experiment for the helimagnons at ambient pressure indicates that the helimagnons are most likely not the source for the NFL behavior of the resistivity above $P_{c}$. 
It is clear that a better understanding of the NFL transport properties above $P_{c}$ may be obtained by revealing the nature of partial magnetic order. Several forms of complex unconventional magnetic order stabilized by means of higher order terms of the DMI have been proposed as possible candidates for the partial order [185-188]. Recently, small angle neutron scattering (SANS) and Hall-effect measurements, in combination with detailed calculations, revealed the existence of a topological magnetic state in $\mathrm{MnSi}$, although at ambient pressure in a small phase pocket just below $T_{C}$ and for small magnetic fields $[189,190]$. The so-called A-phase represents a skyrmion lattice similar to the proposals for the partial order, proving that such a magnetic phase in fact can be stable. A calculation in Ref. [189] showed that the skyrmion lattice may only be stabilized by thermal fluctuations of the magnetization over short length scales. We therefore extended our study of the magnetic excitations at ambient pressure by performing measurements with magnetic fields. The result presented in Fig. 14 show that the magnetic excitations in the A-phase of $\mathrm{MnSi}$ are strongly reminiscent of the helimagnons observed in the helical phase [191]. This establishes helimagnons as a possible prerequisite for complex chiral magnetic textures.

A further candidate for a complex chiral magnetic phase in $\mathrm{MnSi}$, reminiscent of the partial order at high pressure, is currently under discussion. In a small temperature interval of about $1 \mathrm{~K}$ above $T_{C}$ in zero magnetic field, SANS measurements suggest that the helix is unpinned [192, 193], which is, in addition, observed in various bulk measurements [194-197]. An alternative point of view is that the characteristic paramagnetic fluctuations of a helimagnet are observed [193, 198]. By means of polarimetric neutron spin echo measurements, Pappas et al. demonstrated that the chiral fraction of the magnetic fluctuations in this temperature interval does not agree with this scenario and proposed that the observed data may be better explained by a skyrmion liquid phase [199] as suggested by Rößler et al. (see electronic supplement of [185]). However, the proposal lacks a calculation demonstrating that a skyrmion liquid would be (i) a stable phase under the investigated conditions and (ii) would lead to the observed chiral fraction, and the issue remains unresolved.

In summary, the source of the observed extended NFL behavior in MnSi still remains unclear. Currently, there is no theoretical account of how the observed forms of complex chiral magnetic order may induce the NFL resistivity observed in $\mathrm{MnSi}$; further theoretical efforts along this line would be highly desirable. From the experimental point of view, the clear understanding of the Goldstone modes of the helimagnetic ground state at ambient pressure [176] may provide a new route towards the exploration of the unconventional behavior above $P_{c}$. Here, the future challenge consists of tracking the helimagnons as a function of pressure in order to verify that their characteristics change distinctively around $P_{c}$ or if even entirely new excitations are observed. An additional task involves the exact determination of the dynamical nature of the partial order as a function of pressure, temperature and magnetic field.

\section{Ferromagnetic superconductors}

Materials such as $\mathrm{ErRh}_{4} \mathrm{~B}_{4}$ [200-203] and $\mathrm{HoMo}_{6} \mathrm{~S}_{8}$ [204, 205], which display the coexistence of superconductivity and ferromagnetism, have been known for over 
three decades. In the latter compounds, $T_{C}$ lies below $T_{c}$ and the superconductivity vanishes at a second, lower critical temperature $T_{c}^{\prime}<T_{C}$. Within the temperature interval between $T_{c}^{\prime}$ and $T_{C}$, the ferromagnetism and superconductivity coexist macroscopically (in a spatially inhomogeneous manner), whereas a new sinusoidallymodulated state with a wavelength $\sim 100 \AA$ and superconductivity coexist microscopically (within the same volume element) [206]. In contrast, the recently discovered uranium-based compounds $\mathrm{UGe}_{2}$ (under pressure) [207], URhGe [24], UIr (under pressure) [208, 209], and UCoGe [210] appear to exhibit the microscopic coexistence of superconductivity and true itinerant electron ferromagnetism. Such a coexistence is intriguing since, in a conventional superconductor, the large internal field generated by the ferromagnetic order would be expected to destroy the superconducting state by breaking the spin-singlet Cooper pairs [211]. It is thus often suggested that the superconducting electrons in such compounds may pair in triplet states, mediated by critical fluctuations associated with a ferromagnetic quantum critical point (QCP) [210, 212, 213]. Non-Fermi-liquid behavior might be expected in the vicinity of a ferromagnetic QCP for the reasons discussed above.

Although the paramagnetic to ferromagnetic transition is often considered as a prototype of a second order phase transition, it has been proposed that a generic feature of itinerant ferromagnets is that the transition becomes first order when it is suppressed towards zero temperature [214]. This implies that the phase diagrams of the uranium-based ferromagnetic superconductors described below may not, strictly speaking, contain QCPs. This has important implications for our understanding of the mechanism of superconductivity in such compounds, as well as for the presence of NFL behavior. In the case of phase transitions that are only very weakly first order, nearly quantum critical fluctuations may still be present and lead to the formation of novel superconducting states [215]. In the remainder of this section, we review the phase diagrams of uranium-based ferromagnetic superconductors as tuned by doping, magnetic field, and pressure with a focus on the presence, or absence of, NFL behavior. For comparison, we also briefly discuss the phase diagram of the simplest ferromagnet-based superconductor, elemental iron. For each figure, we have attempted to reproduced the published data as accurately as possible, though for the definitive representation, the reader is referred to the literature cited in the figure captions.

Figure $15 \mathrm{a}$ presents the phase diagram of $\mathrm{UGe}_{2}$ under pressure. At ambient pressure, $\mathrm{UGe}_{2}$ displays ferromagnetic order below $T_{C 1}=52 \mathrm{~K}$. Under pressure, this ordering temperature drops monotonically to zero near a critical pressure $P_{c 1} \sim 1.6$ GPa [207]. A second ferromagnetic transition $T_{C 2}$ at lower temperatures is also monotonically suppressed until it is driven to zero near a critical pressure $P_{c 2} \sim$ 1.2 GPa. Just below $P_{c 2}$, superconductivity appears, passing through a dome with maximum critical temperature of $T_{c} \sim 0.9 \mathrm{~K}$ at $P_{c 2}$. The entire dome appears within the ferromagnetic phase and superconductivity does not persist into the paramagnetic region beyond $P_{c 1}$. The complete absence of superconductivity in the paramagnetic phase offers circumstantial evidence that the superconductivity in $\mathrm{UGe}_{2}$ is of an unconventional nature. A careful study of the saturation moment under pressure shows that it changes abruptly and discontinuously near each critical pressure (Fig. 15(d)), and at pressures above the critical pressure, a sufficiently large magnetic field induces 
Fig. 15 Characteristics of $\mathrm{UGe}_{2}$ as a function of pressure. (a) Ferromagnetic ordering temperatures $T_{C 1}$ and $T_{C 2}$, and superconducting critical temperature $T_{c}$ multiplied by 10 [207, 219]. (b), (c) Residual resistivity and $A$ coefficient of power law fit to the electrical resistivity, respectively for polycrystalline $\mathrm{UGe}_{2}$ [21]. (d) Saturation magnetization $\mu_{s}$ and $\mu_{x}$. The value of $\mu_{x}$ is determined by extrapolating the magnetization to zero from above $H_{x}$, the critical field for a metamagnetic transition [216].

(e) Coefficient $\gamma$ of the electronic specific heat for single crystal (open circles) [219] and polycrystalline (filled circles) [220] samples

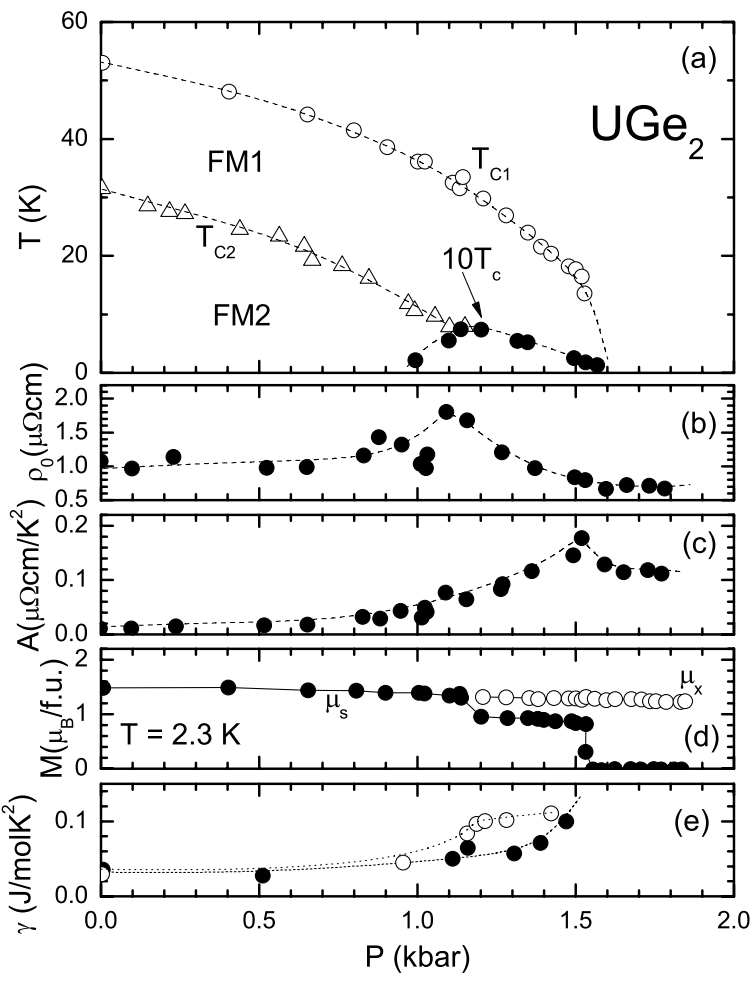

metamagnetic transitions, proving that the transitions become first order [216]. Accordingly, it is likely that the critical pressures in $\mathrm{UGe}_{2}$ represent QPTs rather than QCPs. Nonetheless, the large upper critical field, which exceeds both the paramagnetic and orbital limits for a weak coupled $s$-wave superconductor implies that the superconducting state in $\mathrm{UGe}_{2}$ can not be conventional [217]. The low temperature resistivity follows $T^{2}$ power law behavior over the entire pressure range from ambient to $\sim 2 \mathrm{GPa}[21,207,218]$. Interestingly, while the residual resistivity $\rho_{0}$ (Fig. 15(b)) passes through a maximum near $P_{c 2}$ where $T_{c}$ is also maximized, the $A$ coefficient (Fig. 15(c)) passes through a maximum near $P_{c 1}$ [21]. Specific heat measurements under pressure show that the electronic coefficient of the specific heat $\gamma$ (Fig. 15(e)) increases as the pressure is increased towards $P_{c 1}[219,220]$, consistent with an increase in the electron effective mass near this pressure.

The temperature $T$ vs. magnetic field $H$ phase diagram of single crystalline URhGe is shown in Fig. 16(a). URhGe displays FM below $T_{C} \sim 9.5 \mathrm{~K}$ with the spontaneous magnetization parallel to the $c$-axis, and develops superconductivity below $\sim 0.2 \mathrm{~K}$ [24]. The upper critical field $H_{c 2}$ significantly exceeds the Pauli paramagnetic limiting field which lends support to the idea that URhGe displays spin-triplet pairing [22]. Remarkably, a re-entrant superconducting (RSC) phase appears when a field above $\sim 10 \mathrm{~T}$ is applied along the $b$-axis (perpendicular to the magnetic easy axis) [23]. Applying magnetic fields along the $b$-axis results in the spins progressively tilting away from the $c$-axis towards the $b$-axis. Eventually, a first order transition oc- 
Fig. 16 (a) Temperature $T$ vs. magnetic field $H$ phase diagram of single crystalline URhGe, for fields applied parallel to the $b$-axis (perpendicular to the magnetic easy axis) [23, 224]. $\mathrm{SC}$ and RSC indicate the superconducting and re-entrant superconducting phases respectively. $H_{R}$ indicates the critical field for which the easy magnetization switches from the $c$ - to $b$-axis [224]. The field dependence of the Curie temperature is taken from Ref. [225]. (b) and (c) show representative values of the $\rho_{0}$ and $A$ coefficients, respectively, for fits to the low temperature electrical resistivity with $\rho(T)=\rho_{0}+A T^{2}$ [224].

(d) Total magnetic moment $M_{\text {tot }}$ and moment parallel to the $b$-axis $M_{b}$ for fields applied along the $b$-axis, as measured by neutron scattering [23]
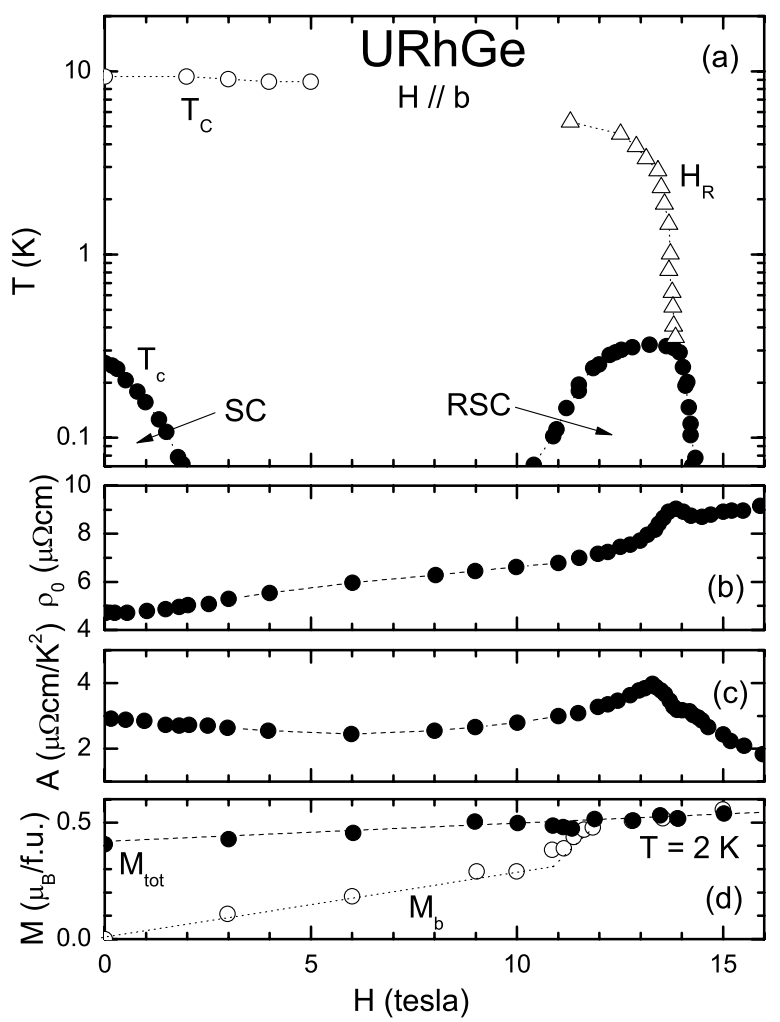

curs and the spins jump into alignment with the $b$-axis at the critical field for spin reorientation $H_{R}$ (see Fig. 16(d)). The RSC phase appears in a dome centered around the extrapolated low temperature value of $H_{R}$ [23]. Thus, the RSC in URhGe appears not to be linked to a prototypical ferromagnetic QCP, but rather to fluctuations associated with a first order transition in the orientation of the magnetic moments. Power law fits to the low temperature electrical resistivity indicate $T^{2}$ Fermi-liquid behavior over the measured field range up to $\sim 16 \mathrm{~T}$ [224]. Both coefficients, $\rho_{0}$ and $A$, pass through maxima near $H_{R}$ (see Figs. 16(b) and 16(c), respectively). Assuming a Kadowaki-Woods relationship, $m^{*} \sim \sqrt{A}$, between $A$ and the effective mass $m^{*}$, one may associate the RSC with an enhancement of the quasiparticle effective mass in the vicinity of $H_{R}$ [224].

A detailed review of the properties of $\mathrm{UCoGe}$ is provided by Gasparini et al. elsewhere in this issue. In the following, we summarize phase diagrams of UCoGe, as tuned by magnetic field, pressure, and doping. UCoGe displays coexisting superconductivity and ferromagnetic order with the superconducting critical temperature $\left(T_{c} \sim 0.8 \mathrm{~K}\right)$ below the Curie temperature $\left(T_{C} \sim 3 \mathrm{~K}\right)$ [210]. NMR measurements show that the ferromagnetic transition in UCoGe is of first-order character [230]. The small saturated moment, $\mu_{\text {sat }} \sim 0.03 \mu_{B}$ [210], and large ratio of the effective moment to saturated moment $\mu_{\text {eff }} / \mu_{\text {sat }}$, indicates the highly itinerant nature of the ferromagnetism. 
Fig. 17 (a) Curie temperature and superconducting critical temperature of UCoGe single crystals as a function of magnetic field applied along the $b$-axis, perpendicular to the easy magnetization axis [231]. (b) $A$ coefficient of quadratic $T^{2}$ fits to the electrical resistivity as a function of fields applied along different crystallographic axes [231]

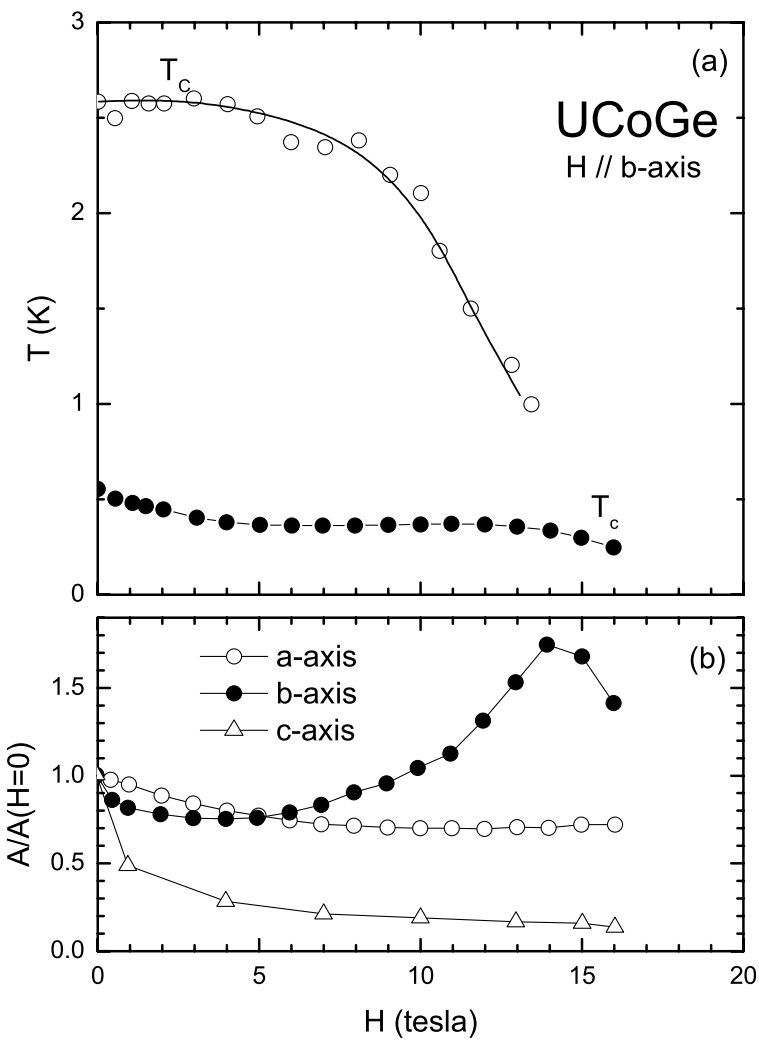

Like URhGe, the magnetic easy axis for the FM in UCoGe is along the $c$-axis. For magnetic fields applied along the $a$-axis of UCoGe, large zero temperature critical fields, $H_{c 2}(0) \sim 20-30 \mathrm{~T}$, are found [231]. For fields aligned to the $b$-axis, hints of re-entrant superconductivity are evinced by an "S"-shaped bend in the upper critical field curve near $T / T_{c} \sim 0.65$ (Fig. 17(a)). $T_{C}$ is suppressed with increasing magnetic field and is destroyed near $15 \mathrm{~T}$. This is near the same field at which there appears to be a small enhancement of the critical temperature. The temperature vs. field phase diagram of UCoGe for fields applied along the $b$-axis is reminiscent of that observed in URhGe. However, for UCoGe at intermediate magnetic fields, the superconductivity is not completely suppressed to zero as it is for URhGe in the field region between the SC and RSC phases. A similar "S"-shaped bend in the upper critical field curve is also found for $\mathrm{UGe}_{2}$ [217]. For all field orientations and fields (up to $16 \mathrm{~T}$ ), the resistivity can be fit by a Fermi liquid power law with $n=2$. However, because $T_{C}$ and $T_{c}$ are rather close together, the temperature interval over which the fitting is performed is very small (this is also an issue for the power law fits described below for UCoGe under pressure and Si-doped UCoGe). For fields along the $a$ - and $c$-axes, the coefficient $A$ is gradually suppressed, while for fields applied along the $b$ axis, $A$ passes through a maximum near the field (15 T) at which FM order is destroyed [231] (Fig. 17(b)). This suggests the effective mass $m^{*}$ is increased at a field-induced QCP near $15 \mathrm{~T}$, which could contribute to the enhancement of the superconductivity. 
Fig. 18 (a) Pressure dependence of the Curie temperature $T_{C}$ and superconducting critical temperature $T_{c}$ for both polycrystalline [25] and single crystalline [26] UCoGe. Panels (b) and (c) show the results of quadratic $T^{2}$ fits to the electrical resistivity over the narrow temperature window between $T_{C}$ and 1.7 K. (d) Magnitude of the feature at $T_{C}$ in the ac magnetic susceptibility signal, which may be related to the size of the ordered magnetic moment

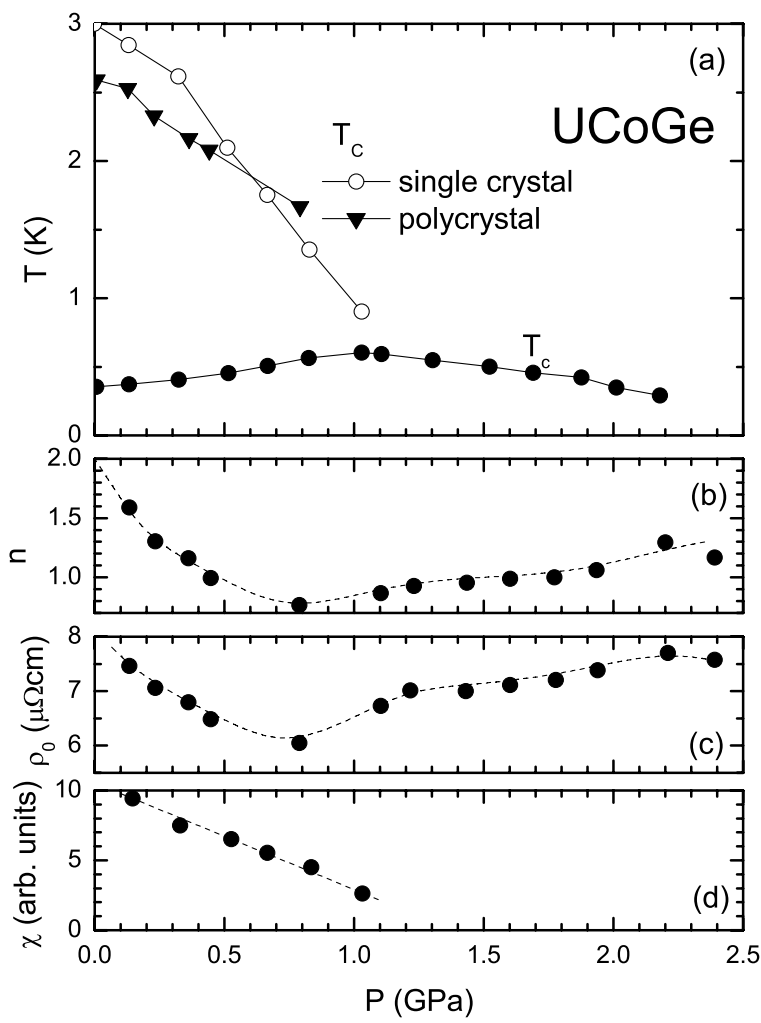

The effect of pressure on some properties of UCoGe is illustrated in Fig. 18. Under pressure, $T_{C}$ decreases and $T_{c}$ initially increases $[25,26]$. Near $1-1.5 \mathrm{GPa}, T_{C}$ is suppressed to zero temperature and $T_{c}$ passes through a weak maximum. The magnitude of the feature in the ac magnetic susceptibility at $T_{C}$, which may be related to the size of the ordered moment, is also gradually suppressed with pressure and extrapolates to zero near $P_{c} \sim 1.5 \mathrm{GPa}$. The exponent $n$ drops from $n=2$ at ambient pressure to $n \lesssim 1$ near the critical pressure (Fig. 18(b)). At higher pressures, $n$ begins a slow recovery towards higher values, although by $2.5 \mathrm{GPa}$ (the highest pressure reported), $n$ has only reached $\sim 1.25$. Surprisingly, $\rho_{0}$ passes through a minimum near this critical pressure. At the critical pressure, one also finds a peak in the coefficient $A$ (not shown), although the interpretation of $A$ for a non-quadratic temperature dependence is unclear. As mentioned above, these coefficients of the power law behavior are based on fitting over a quite small temperature range [25]. Although there is evidence for a pressure-induced QCP in UCoGe, as provided by the gradual suppression of the magnetic state and perhaps the appearance of NFL behavior in the electrical resistivity, the superconductivity seems to persist over a broad range of pressures, rather than being localized around the putative quantum critical point. Furthermore, the superconducting state seems to be unperturbed by the transition from ferromagnetism to paramagnetism. 
Fig. 19 (a) Curie temperature $T_{C}$ and superconducting critical temperature $T_{c}$ as a function of dopant concentration $x$ in $\mathrm{UCoGe}_{1-x} \mathrm{Si}_{x}$ [212]. (b) Exponent $n$ obtained from power law fits to the low temperature electrical resistivity [212]. (c) Saturated moment as determined by extrapolating the ordered magnetization to zero field [212]
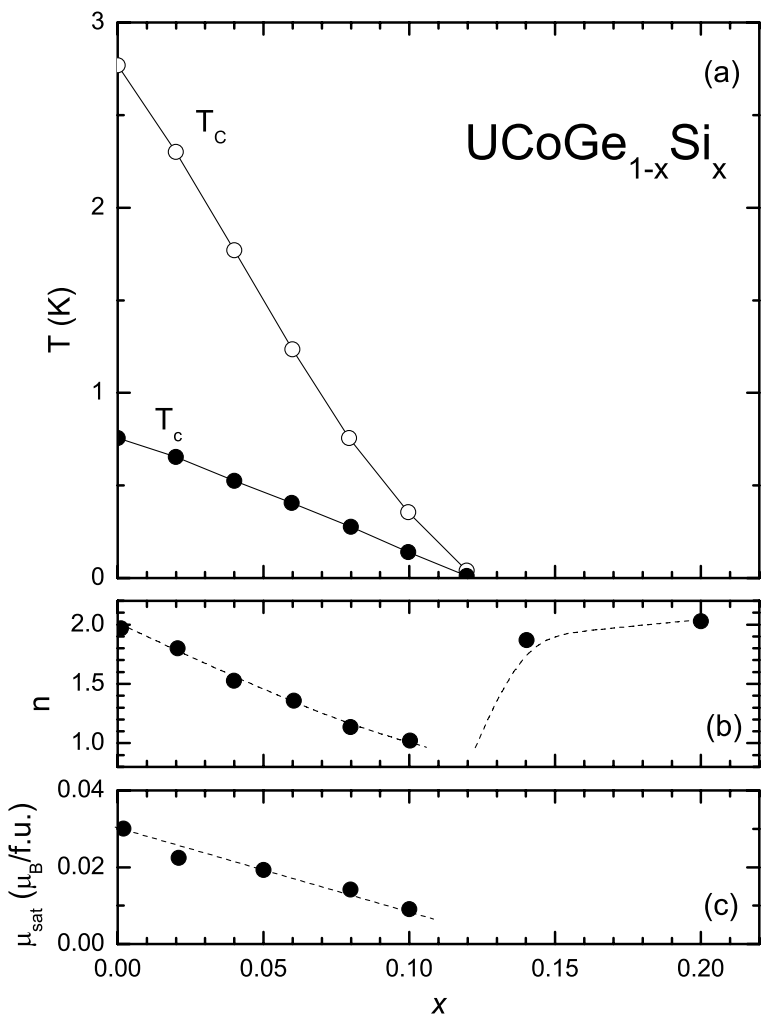

Upon substituting $\mathrm{Si}$ for Ge (UCoGe $\left.{ }_{1-x} \mathrm{Si}_{x}\right)$, both $T_{c}$ and $T_{C}$ decrease until they both vanish simultaneously for $x \gtrsim 0.12$ [212] (Fig. 19). One might expect that this kind of isovalent replacement of Ge with the smaller Si ion would result in a "chemical pressure" that would mimic the effect of physical pressure. However, chemical substitution has the additional effect of introducing atomic disorder, which may be the reason behind the monotonic suppression of $T_{c}$. The fact that the superconductivity and ferromagnetism appear to vanish at the same critical concentration, suggests an intimate link between the two states, wherein the superconductivity is enhanced by the presence of ferromagnetic order. The low temperature resistivity follows powerlaw behavior throughout the doping range $0-0.2$ with the exponent $n$ dropping from $\sim 2$ in the undoped compound to $\sim 1$ at $x=0.1$ near the critical concentration for the destruction of ferromagnetism. The exponents for concentrations below $x=0.12$ are based on fitting the electrical resistivity only over the small temperature interval between $T_{c}$ and $T_{C}$. At high silicon concentrations, Fermi-liquid $n=2$ power law behavior is regained.

We have studied the effect of doping from ferromagnetic UCoGe to paramagnetic UFeGe. UFeGe exhibits a monoclinic distortion of the orthorhombic TiNiSi structure below $\sim 500^{\circ} \mathrm{C}$. However, we found that the orthorhombic TiNiSi structure of UCoGe persisted up to $x \lesssim 70 \%$ for $\mathrm{UCo}_{1-x} \mathrm{Fe}_{x} \mathrm{Ge}$. Similar to a recent report which was restricted to small dopant concentrations [232], we found that the Curie tempera- 
Fig. 20 (a) Curie temperature $T_{C}$ and superconducting critical temperature $T_{c}$ as a function of dopant concentration $x$ in $\mathrm{UCo}_{1-x} \mathrm{Fe}_{x}$ Ge [234].

(b) Saturated moment as determined by extrapolating the ordered magnetization to zero field. (c) Effective magnetic moment from Curie-Weiss fits to the temperature dependent magnetic susceptibility.

(d) Ratio of the effective moment to saturation moment indicating a highly itinerant ferromagnetic state near $x=0.2$
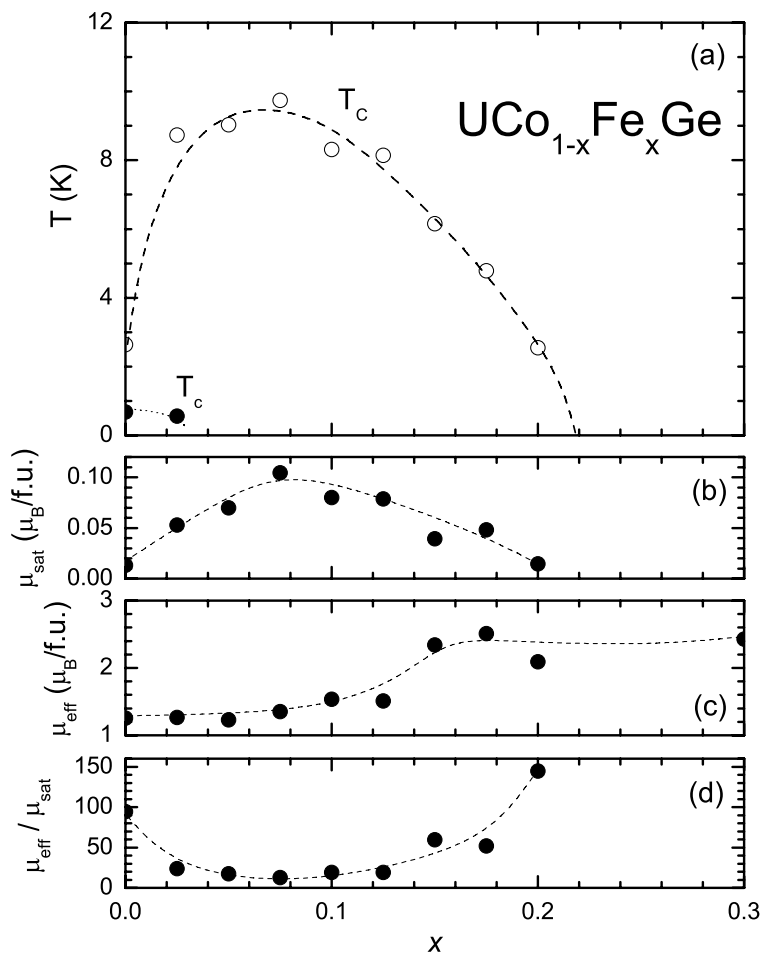

ture initially increased and the superconductivity was rapidly suppressed (Fig. 20(a)). At higher iron concentrations, the Curie temperature passes through a maximum and is then suppressed to zero near $x_{c}=0.22$. The saturation moment, as determined by extrapolating the ordered magnetization to zero, also passes through a maximum and vanishes near $x_{c}$ (Fig. 20(b)). The effective moment, estimated by fitting the temperature dependent magnetic susceptibility to Curie-Weiss behavior, exhibits an increase with doping (Fig. $20\left(\right.$ c)). The ratio $\mu_{\text {eff }} / \mu_{\text {sat }}$ of the effective moment to the saturation moment, which offers a measure of the degree of itinerancy, passes through a minimum near the concentration at which $T_{C}$ is maximized (Fig. 20(d)) and then increases at higher $x$. At $x_{c}$, the ratio $\mu_{\text {eff }} / \mu_{\text {sat }}$, is even larger than in undoped UCoGe, indicating a high degree of itinerancy. Thus, there is some good evidence for a QCP near $x_{c}$. If the superconductivity in UCoGe is indeed spin-triplet in nature, the lack of superconductivity near the $\mathrm{UCo}_{0.8} \mathrm{Fe}_{0.2} \mathrm{Ge}$ concentration may be a result of the atomic disorder inherent in doped samples. Efforts are currently underway to study the region near $\mathrm{UCo}_{0.8} \mathrm{Fe}_{0.2} \mathrm{Ge}$ with finer doping increments, in high quality singleand polycrystalline samples, in order to investigate whether superconductivity can be induced near this FM QCP. In a recent extension of this work, we have found that NFL temperature dependences of the electrical resistivity and specific heat develop in samples close to the $x_{c}$ concentration [233].

Figure 21 presents data for UIr as a function of pressure. UIr is an Ising-type ferromagnet with a Curie temperature $T_{C 1} \sim 46 \mathrm{~K}$ and the spin easy axis along [101] . The small ratio of the ordered moment $\left(0.5 \mu_{B} / U\right)$ to the effective moment $\left(2.4 \mu_{B} / U\right)$ 
Fig. 21 (a) Temperature $T$ vs. pressure $P$ phase diagram for UIr. The open circles indicate the various FM ordering temperatures as measured by electrical resistivity, ac magnetic susceptibility, and magnetization [226]. The superconducting critical temperature $T_{c}$ has been multiplied by a factor of 50 . (b), (c), and (d) show the parameters given by fitting the low temperature electrical resistivity of single crystalline samples with

$\rho(T)=\rho_{0}+A T^{n}$ [208].

(e) Pressure dependence of the ordered magnetic moment for two different samples, as determined by either residual magnetization or Arrott plot analysis [226]

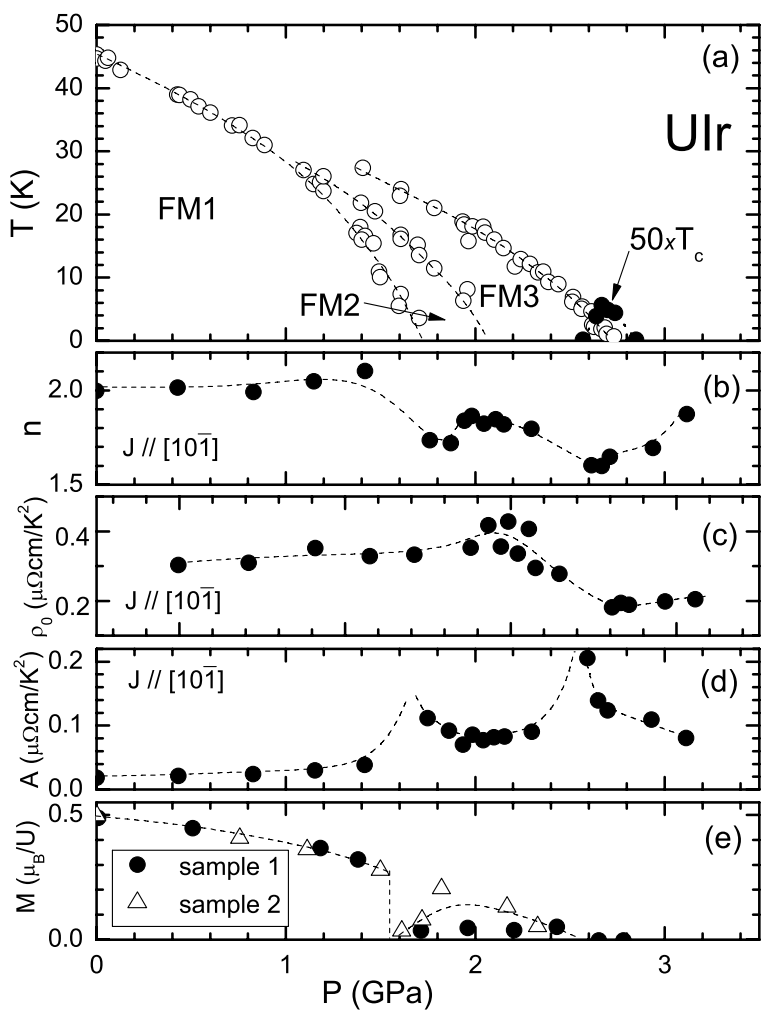

indicates the itinerant nature of the ferromagnetism. Under pressure, $T_{C 1}$ drops monotonically [227] and then vanishes at the critical pressure $P_{C 1} \sim 1.7$ GPa [209]. A second ferromagnetic phase, FM2 appears above $\sim 1.2 \mathrm{GPa}$. The ordering temperature of the FM2 phase is monotonically suppressed with pressure from $\sim 30 \mathrm{~K}$ near $1.2 \mathrm{GPa}$ and vanishes abruptly near the critical pressure $P_{C 2} \sim 2 \mathrm{GPa}$. Yet a third magnetic transition appears above $\sim 1.4 \mathrm{GPa}$ at $\sim 30 \mathrm{~K}$ and is monotonically suppressed continuously to zero temperature near $P_{c 3} \sim 2.7 \mathrm{GPa}$. Superconductivity with a maximum temperature $T_{c} \sim 0.14 \mathrm{~K}$ appears in a very narrow dome localized around $P_{C 3}$ [208]. The superconductivity has been observed by zero electrical resistance and by a drop in the ac magnetic susceptibility [226, 228]; however, thermodynamic signatures of the superconducting transition (via, e.g., ac calorimetry) have not, to our knowledge, been reported. An interesting feature of UIr is that the crystal structure is non-centrosymmetric. The presence of superconductivity in a ferromagnetic material which lacks inversion symmetry raises some interesting questions about the symmetry of the superconducting order parameter [229]. Figure 21(e) displays the pressure dependence of the ordered moments for the various ferromagnetic phases as a function of pressure, as determined by residual magnetization or Arrott plot analysis [208, 226]. Within the FM1 phase, the ordered moment decreases monotonically with pressure and just below $P_{C 1}$ has dropped to roughly half of the ambient pressure value. Upon further increase of pressure, the ordered moment drops abruptly in the 
Fig. 22 (a) Structural, magnetic, and superconducting phases of iron as a function of pressure [20, 31, 221]. The superconducting critical temperature has been multiplied by a factor of 100 to make it more visible. (b), (c), and (d) show the $n, \rho_{0}$, and $A$ coefficients of power law fits to the electrical resistivity, respectively $[30,31]$

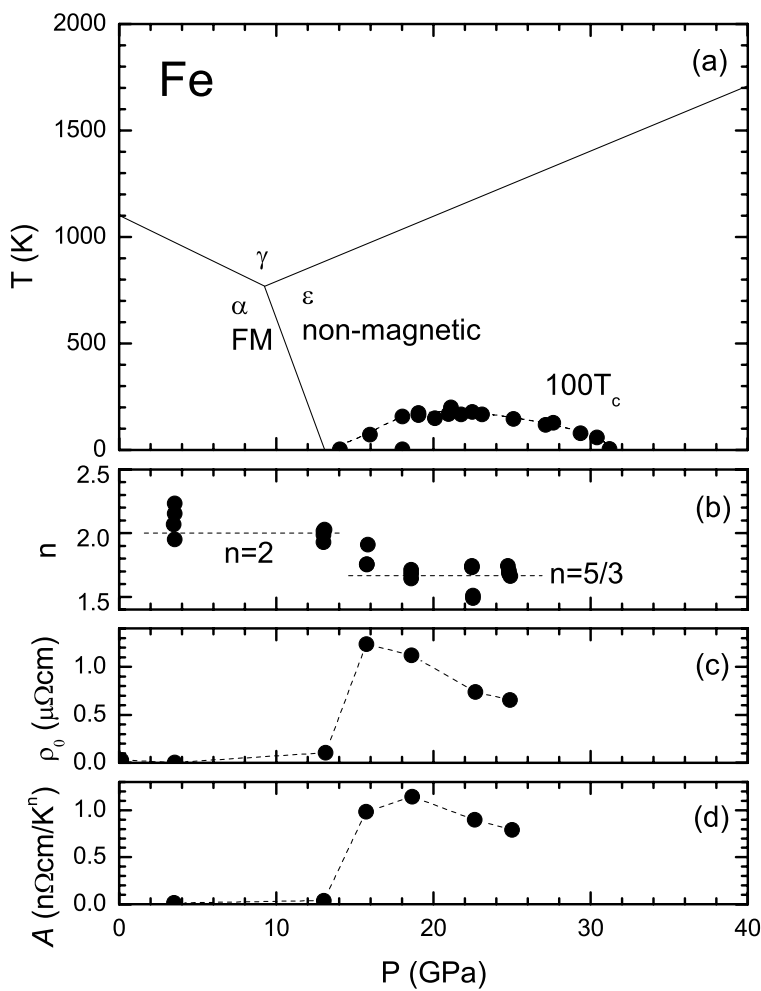

FM2 phase to a much smaller value of $\lesssim 0.08 \mu_{B} / U$. In the FM3 phase, two different samples exhibited rather different values of the ordered moment; however, for both samples the ordered moment decreases with pressure and vanishes above $P_{C 3}$. At pressures below $P_{C 1}$, the low temperature electrical resistivity is well described by Fermi-liquid $T^{2}$ power law behavior (Fig. 21). Above $P_{C 1}$, the exponent of the power law abruptly drops to $n \sim 5 / 3$, corresponding to the predicted behavior due to critical fluctuations of a three-dimensional ferromagnet. Just above $P_{C 1}$, the $n \sim 5 / 3$ power law behavior persists over more than two decades in temperature, from $0.1-$ $15 \mathrm{~K}$. Upon further increase of pressure, $n$ increases weakly and then passes through another minimum of $n \sim 5 / 3$ at $P_{C 3}$. Above $P_{C 3}$, the exponent begins to recover towards $n=2$. The residual resistivity passes through a maximum in the vicinity of the FM2 $\rightarrow$ FM3 transition (Fig. 21(c)). The $A$ coefficient (Fig. 21(d)), determined by fitting the lowest temperature data with a $T^{2}$ power law, passes through maxima near $P_{C 1}$ and $P_{C 3}$, the same pressures at which the NFL behavior is most pronounced. It is interesting to note that the critical pressures $P_{C 1}$ and $P_{C 3}$ are quite similar in terms of their NFL behavior, but that superconductivity only develops at the critical pressure associated with a $\mathrm{FM} \rightarrow \mathrm{PM}$ transition and not the one associated with the transition between two different FM phases.

As shown in Fig. 22(a), iron converts from a ferromagnetic $b c c$ " $\alpha$ " phase to a non-magnetic $h c p$ " $\epsilon$ " structure at pressures above $\sim 10 \mathrm{GPa}$ (at low temperature). Recent combined XAS and XMCD measurements indicate that the magnetic phase 
transition slightly precedes the structural transition, suggesting that the magnetic transition drives the structural one [222]. The sharpness of the transitions is taken as evidence that both transitions are first order. At pressures above that required to drive iron into the non-magnetic $\epsilon$ phase, superconductivity appears and passes through a broad maximum of $\sim 2 \mathrm{~K}$ near $20 \mathrm{GPa}$ [221]. The fact that the superconductivity only appears in the non-magnetic phase (exactly the opposite of the situation for $\mathrm{UGe}_{2}$ ) might lead one to conclude that iron is a conventional superconductor since the onset of ferromagnetism immediately destroys the superconductivity. In addition, the first order character of the magnetic and structural transitions would seem to rule out the presence of strong critical fluctuations in the vicinity of the $\alpha \rightarrow \epsilon$ transition as the driving force behind the superconductivity. Nonetheless, at least one piece of evidence suggests an unconventional pairing mechanism in iron: the superconductivity is highly sensitive to non-magnetic disorder, which is an expected feature of spin-triplet superconductors. Complete resistive transitions are only observed in samples with an electronic mean free path above a certain threshold value [30]. NFL behavior is observed in the exponent $n$ of power law fits to the normal state electrical resistivity, which displays an abrupt change from $n \sim 2$ at pressures below the $\alpha \rightarrow \epsilon$ transition, to $n \sim 5 / 3$ at pressures above the $\alpha \rightarrow \epsilon$ transition [30, 31] (Fig. 22(b)). The $T^{5 / 3}$ power law behavior is expected within the nearly ferromagnetic Fermi-liquid model [223]. It is remarkable that this NFL power law behavior persists far into the non-magnetic state, to pressure at least twice that required to drive the system nonmagnetic. Thus, iron appears to exhibit an extended NFL quantum phase, rather than a small region of NFL behavior localized around a QCP.

It appears that NFL behavior is absent in the above phase diagrams when the ordered moment jumps or vanishes discontinuously at the critical value of the tuning parameter. This seems to be the case for both $\mathrm{UGe}_{2}$ and $\mathrm{URhGe}$, and is consistent with the transitions in these materials having a stronger first order character. In UCoGe (and possibly UIr), where the ordered moment extrapolates to zero near the critical value of the tuning parameter, as would be expected for a true QCP, NFL behavior may be observed. The situation in pure iron is intriguing, in that the $\alpha \rightarrow \epsilon$ magnetostructural transition is strongly first order, yet the transition is accompanied by the appearance of NFL behavior.

\section{$8 \mathrm{Yb}_{2} \mathrm{Fe}_{12} \mathbf{P}_{7}$}

Yb-based compounds that exhibit heavy fermion and NFL behavior are quite rare. In this section, we discuss the unconventional $T-H$ phase diagram in the compound $\mathrm{Yb}_{2} \mathrm{Fe}_{12} \mathrm{P}_{7}$. For comparison purposes, we first briefly review the behavior of the well studied compound $\mathrm{YbRh}_{2} \mathrm{Si}_{2}$, which is thought to be a prototypical example of the Kondo destruction QCP scenario where antiferromagnetic order is suppressed to $T=0 \mathrm{~K}$ by a magnetic field, accompanied by a crossover from a small to a large Fermi surface; i.e., a heavy Fermi liquid emerges for $\delta_{c} \leq \delta$. In this instance, the suppression of the ordered state and the so-called Kondo destruction occur at the same $\delta$, in contrast with the spin density wave scenario. Order parameter fluctuations are manifested as NFL behavior at $T \mathrm{~s}$ above the QCP and a line emanating from the QCP 
delineates a gradual crossover from NFL to FL behavior at lower $T$ and higher values of $\delta$.

The compound $\mathrm{YbRh}_{2} \mathrm{Si}_{2}$ has attracted intense interest because it is a model example of a heavy fermion (HF) system where NFL effects are observed near the suppression of a magnetic ordering temperature [47, 235, 236]. This material exhibits high crystalline purity, is stoichiometric, and is found extremely close to (but still on the magnetic side of) a QCP. Moreover, it was the first example of a Ybbased compound to show strong evidence for NFL behavior near a QCP. Similar to $\mathrm{CeCu}_{2} \mathrm{Si}_{2}$, it crystallizes in a tetragonal structure $(I 4 / \mathrm{mmm})$ with lattice constants $a=4.007 \AA$ and $c=9.858 \AA$. The high temperature magnetic susceptibility $\chi(T)$ for both crystallographic directions reveals Curie-Weiss (CW) behavior (for $200 \mathrm{~K}$ $<T$ ) with the effective magnetic moment $\mu_{\mathrm{eff}} \approx 4.5 \mu_{B} / \mathrm{Yb}$ and $\mathrm{CW}$ temperatures $\theta=-9 \mathrm{~K}$ and $-180 \mathrm{~K}$ for magnetic field parallel and perpendicular to the $c$-axis, respectively. The electrical resistivity is nearly constant for $100 \mathrm{~K}<T$. As expected near the onset of a coherent ground state, $\rho(T)$ decreases precipitously below the coherence temperature $T_{c o h}$. Measurements of $\chi_{a c}(T), \rho(T)$, and $C(T) / T$ reveal a phase transition to a magnetic ground state near $T_{M} \approx 65 \mathrm{mK}$ which is thought to be antiferromagnetic, although bulk magnetization measurements indicate ferromagnetic fluctuations above the QCP [237]. Measurements for $\mathrm{YbRh}_{2}\left(\mathrm{Si}_{1-x} \mathrm{Ge}_{x}\right)_{2}$ $(x=0.05)$ show that isoelectronic substitution suppresses $T_{M}$ below $20 \mathrm{mK}$ [238].

Early measurements also revealed deviations from $\mathrm{FL}$ behavior in $\mathrm{YbRh}_{2} \mathrm{Si}_{2}$ near the QCP including: A linear $T$-dependence of $\rho(T)$ for $20 \mathrm{mK}-1 \mathrm{~K}$, a logarithmic $T$-dependence of $C(T) / T$ for $0.3 \mathrm{~K}-10 \mathrm{~K}$, and a power law dependence of $C(T) / T$ for $T<0.3 \mathrm{~K}[47,235,238]$. Inelastic neutron scattering experiments showed that these types of behavior are unrelated to the excited crystalline electric field states [239]. By analogy to $\mathrm{CeCu}_{6-x} \mathrm{Au}_{x}$, these results were taken as evidence for 2D antiferromagnetic critical fluctuations near the QCP [47, 236], although it should be pointed out that this conclusion remains an open issue. Subsequently, several other unusual phenomena were observed near the QCP including divergent Grüneisen ratios for $\mathrm{YbRh}_{2}\left(\mathrm{Si}_{0.95} \mathrm{Ge}_{0.05}\right)_{2}$ [240] and further evidence for ferromagnetic quantum critical fluctuations (inferred from ${ }^{29} \mathrm{Si}$ NMR and ESR measurements $[241,242])$. In order to further probe the low $T$ NFL behavior, extensive studies in applied magnetic fields have been performed. For instance, a $T-H$ phase diagram developed from $\rho(T, H)$ data (for $H$ applied parallel to the magnetically hard $c$-axis) is shown in the lower left panel to Fig. 24 where the color gradient represents the evolution of the exponent $n$ where $n(T, H)=\partial \ln \left(\rho-\rho_{0}\right) / \partial \ln T$, assuming $\rho(T, H)=\rho_{0}+A T^{n}$ [238]. As expected for the typical "V" shaped QCP phase diagram, order parameter fluctuations are manifested as NFL behavior at $T$ s above the QCP and a line emanating from the QCP delineates a gradual crossover from NFL to FL behavior at lower $T$ and higher values of $H$. FL behavior $\left(\Delta \rho(T) \sim T^{2}\right)$ is observed inside the magnetically ordered state. Hall effect measurements have also shown that, with increasing field, the Hall coefficient $\left(R_{H}\right)$ decreases abruptly at the AFM QCP. This indicates a sudden increase in the Fermi surface, consistent with the abrupt expansion of the large Fermi surface associated with a heavy fermion state at $H=H_{c}$ [243]. It is noteworthy that the abrupt change is seen only in the low $T$ limit. Results from thermopower measurements were also found to support this point 
of view [244]. These observations have led to the conclusion that the heavy fermion state breaks down at the QCP, commensurate with the suppression of the magnetic state, resulting in quantum fluctuations [245]. As a result, $\mathrm{YbRh}_{2} \mathrm{Si}_{2}$ is thought to be a model example for NFL behavior in the context of an AFM QCP scenario.

We recently reported new measurements on single crystals of the ternary compound $\mathrm{Yb}_{2} \mathrm{Fe}_{12} \mathrm{P}_{7}$ [246]. This material is a member of a broad class of pnictogen-based systems with noncentrosymmetric structures and the chemical formula $L n_{n(n-1)} T_{(n+1)(n+2)} M_{n(n+1)+1}$, where $L n$ is a lanthanide (or actinide), $T$ is a transition metal, and $M$ is a metalloid (phosphorus, arsenic) [247, 248]. The $L n_{2} \mathrm{Fe}_{12} \mathrm{P}_{7}$ compounds crystallize in a noncentrosymmetric hexagonal structure (space group $P \overline{6}$ ) with two $L n$ sites. For $L n=\mathrm{Yb}$, the lattice parameters are $a=9.111 \AA$ and $c=3.626 \AA$. The $\chi(T)$ data reveal Curie-Weiss $(\mathrm{CW})$ behavior where the effective magnetic moments $\left(\mu_{\mathrm{eff}}=4.1 \mu_{B} / \mathrm{Yb}\right.$ and $4.8 \mu_{B} / \mathrm{Yb}$ for $H \| c$ and $H \perp c$, respectively) are close to the value expected for $\mathrm{Yb}^{3+}$ free ions ( $\left.\mu_{\mathrm{eff}}=4.53 \mu_{B} / \mathrm{Yb}\right)$ according to Hund's rules. The crystalline anisotropy is reflected in the CW temperatures $(\theta)$ taken from fits to the data, for which $\theta=1 \mathrm{~K}$ and $-27 \mathrm{~K}$ for $H \| c$ and $H \perp c$, respectively (Fig. 23(c)). For $\chi_{\perp}(T)$, a CW law is observed down to $T \sim 30 \mathrm{~K}$, below which the data increase more weakly than expected from the high $-T \mathrm{CW}$ behavior. The departure from $\mathrm{CW}$ behavior occurs near

Fig. 23 (a) Electrical resistivity $\rho$ vs. temperature $T$ for $T=0.05 \mathrm{~K}-290 \mathrm{~K}$ and $H=0$ T. (a) Inset: $\rho-\rho_{0}$ vs. $T$ on a log-log plot. The fits are described in the text. (b) Specific heat divided by temperature $C / T$ vs. $T$ for several magnetic fields $H$. (b) Inset: Derivative of the electrical resistivity with respect to $T$, $\partial \rho(T, H) / \partial T$. (c) Magnetic susceptibility $\chi=M / H$ vs. temperature $T$ for magnetic field $H$ applied perpendicular and parallel to the $c$-axis. The solid lines represent Curie-Weiss fits to the data as described in the text. (c) Inset: $\chi=M / H$ vs. temperature $T$ for magnetic field $H$ applied perpendicular to the $c$-axis. The solid and dotted lines represent the power law fit and logarithmic fits to the data, respectively, as described in the text

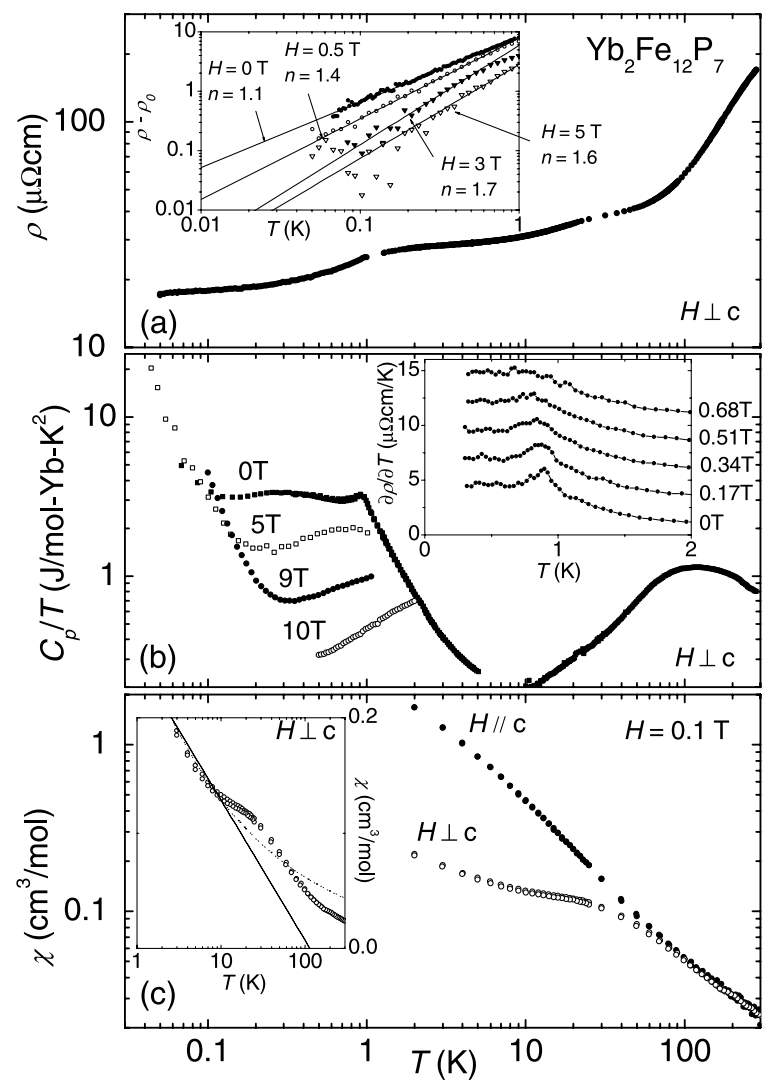




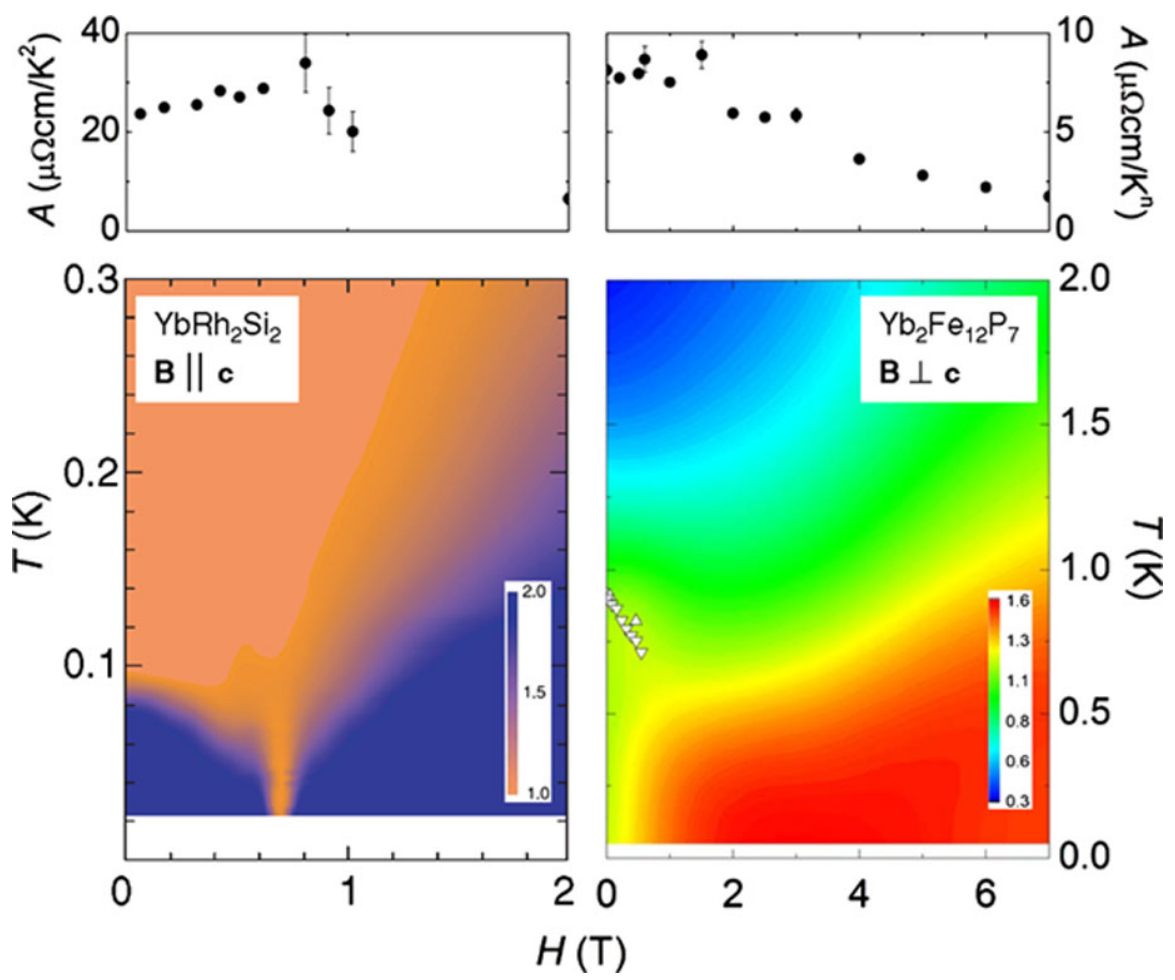

Fig. 24 (Color online) The $T-H$ phase diagrams for $\mathrm{YbRh}_{2} \mathrm{Si}_{2}$ (left panel) and $\mathrm{Yb}_{2} \mathrm{Fe}_{12} \mathrm{P}_{7}$ (right panel). For $\mathrm{Yb}_{2} \mathrm{Fe}_{12} \mathrm{P}_{7}$, the values for $T_{M}$ are taken from measurements of $C(T, H)$ (triangles) and $\rho(T, H)$ (upside down triangles). The color gradients represents the quantity $\partial \ln \left(\rho-\rho_{0}\right) / \partial \ln T$ where $\rho_{0}$ is the value, for each $\rho(T, H)$, that maximizes the temperature range for the low $T$ power law behavior. We emphasize that the values of $\partial \ln \left(\rho-\rho_{0}\right) / \partial \ln T$ above the low $T$ fit range do not represent the true local exponent $n$ since they refer to the value of $\rho_{0}$ from the low $T$ power law behavior. Also note that the temperature range for the low $T$ power law behavior appears to be somewhat reduced from what would be inferred from Fig. 23. This is because it is necessary to smooth $\rho(T)$ before taking the logarithmic derivative. This process has the effect of reducing the apparent fit range because, in the upper temperature limit of the power law region, adjacent averaging samples the higher $T$ data which have a lower local slope than the low $T$ power law

the onset of correlated electron phenomena inferred from $C(T, H)$ and $\rho(T, H)$. A similar trend is observed for $\chi_{\|}(T)$ for $T<10 \mathrm{~K}$. The $\rho(T, H=0)$ data decrease from room $T$, indicating metallic behavior down to $T \sim 50 \mathrm{~K}$. Below $T \sim 30 \mathrm{~K}$, $\rho(T, H=0)$ evolves through a pronounced shoulder (Fig. 23(a)). The $H=0 \mathrm{~T}$ specific heat divided by temperature $C(T, H=0) / T$ data increase rapidly for $T<10 \mathrm{~K}$, suggesting the formation of a strongly correlated electron ground state (Fig. 23(b)). At low $T$, measurements of $\rho(T)$ and $C(T) / T$ reveal a phase transition to a magnetic ground state near $T_{M} \approx 0.9 \mathrm{~K}$, which appears to be antiferromagnetic (Fig. 23).

Low $T$ measurements for $\mathrm{Yb}_{2} \mathrm{Fe}_{12} \mathrm{P}_{7}$ indicate NFL behavior. We find that for $T$ $<10 \mathrm{~K}, \chi_{\perp}(T)$ can be described using either power law or logarithmic functions of the forms $\chi_{\perp}(T)=a T^{-n}$ where $a=0.27 \mathrm{~cm}^{3} \mathrm{~K}^{n} / \mathrm{mol}$ and $n=0.32$ or $\chi_{\perp}(T)=$ $b-c \ln T$ where $b=0.25 \mathrm{~cm}^{3} / \mathrm{mol}$ and $c=0.05 \mathrm{~cm}^{3} / \mathrm{mol}$ (inset Fig. 23(c)). Re- 
markably, $\rho(T, H=0)$ is nearly linear for more than a decade in $T$ below $T_{M}$ (inset Fig. 23(a)). This result is particularly interesting because it implies the existence of an NFL phase deep within the magnetically ordered state which has an associated large value of the electronic specific heat coefficient $\gamma=C(T) / T$. This result is in contrast to typical NFL systems (e.g., $\mathrm{CeCu}_{6-x} \mathrm{Au}_{x}$ and $\mathrm{YbRh}_{2} \mathrm{Si}_{2}$ ) where the NFL behavior emerges only near the QCP and is often commensurate with the destruction of the HF ground state.

Studies of $\rho(T, H)$ and $C(T, H) / T$ were performed in order to develop a $T-H$ phase diagram at low $T$. It is possible to track the evolution of the power law behavior in $\rho(T, H)$ at low $T$ by fitting the data with the expression $\rho(T)=\rho_{0}+A T^{n}$. The best fits were determined from a plot of $\ln \left(\rho-\rho_{0}\right)$ vs. $\ln (T)$ in which the value of $\rho_{0}$ was selected to maximize the linear region of the fit extending from low $T$. Based on this analysis, we find the $T-H$ phase diagram shown in the right lower panel to Fig. 24. First, $T_{M}$ (inferred from $\partial \rho(T, H) / \partial T$ and $C(T, H) / T$ ) decreases with $H$ until it becomes impossible to track for $0.7 \mathrm{~T}<H$. As a result, it is unclear whether there is a first order transition near $0.7 \mathrm{~T}$ or a second order transition in the vicinity of $\sim 1.5 \mathrm{~T}$, where we have extrapolated $T_{M}=0 \mathrm{~K}$ (dashed line in Fig. 24, right panel). From the power law fits to $\rho(T, H)$, we find that $n \sim 1.1$ for $H=0 \mathrm{~T}$. As $H$ increases, $n$ rapidly crosses over to a value $\sim 1.5$ near $H=0.5 \mathrm{~T}$, close to the field where $T_{M}$ is no longer observable. Starting near $H \sim 2.5 \mathrm{~T}$, the $n \sim 1.5$ dependence extends over an increasingly broad $T$ region. From this result, we infer that $\mathrm{Yb}_{2} \mathrm{Fe}_{12} \mathrm{P}_{7}$ has an unconventional ground state which includes a QPT between two phases that exhibit NFL behavior with different power law exponents of the electrical resistivity. On the other hand, we also find that for $1 \mathrm{~T}<H$, the low $T$ value for $C(T, H) / T$ is suppressed and the location of the broad maximum shifts towards higher $T$ (Fig. 23(b)). This behavior is consistent with the recovery of a FL state due to the increase of the Zeeman energy with $H$. This apparent disagreement between $\rho(T, H)$ and $C(T, H) / T$ highlights the point that the $T-H$ phase diagram for $\mathrm{Yb}_{2} \mathrm{Fe}_{12} \mathrm{P}_{7}$ is highly anomalous and does not conform to the classical QCP scenario.

\section{$9 \mathrm{PrOs}_{4} \mathrm{Sb}_{12}$}

The filled skutterudites are a well known class of compounds that have generated intense activity during the past three decades due to the wide variety of interesting electronic and magnetic phenomena that they exhibit and their promising thermoelectric properties [249-251]. Filled skutterudites have the chemical formula $M T_{4} X_{12}$ where the "filler" atom $M=L a-\mathrm{Nd}, \mathrm{Sm}, \mathrm{Eu}, \mathrm{Yb}, \mathrm{U}$, Th, the transition metal $T=\mathrm{Fe}, \mathrm{Ru}$, Os, and the pnictogen $X=\mathrm{P}$, As, Sb. The filled skutterudites exhibit many types of correlated electron behavior including: Spin fluctuations, itinerant electron ferromagnetism, local moment ferromagnetism and antiferromagnetism, conventional BCS (Bardeen-Cooper-Schrieffer) superconductivity, unconventional superconductivity, heavy fermion behavior, hybridization gap semiconducting behavior, nonFermi liquid (NFL) behavior, etc.

The Pr-based filled skutterudites ( $\operatorname{Pr} T_{4} X_{12} ; T=\mathrm{Fe}, \mathrm{Ru}, \mathrm{Os}$ and $X=\mathrm{P}, \mathrm{Sb}, \mathrm{As}$ ) are mainly correlated electron metals which exhibit a variety of low temperature phe- 
Fig. 25 (Color online) The $H-T$ phase diagram for $\mathrm{PrOs}_{4} \mathrm{Sb}_{12}$. The superconducting state phase boundary is derived from the electrical resistivity data. The high field ordered phase (HFOP) is deduced from features observed in $\rho(T, H), C(T, H)$, $M(T, H)(H$ parallel to [001] and [111]), thermal expansion $\alpha(T, H)$, and magnetostriction $\lambda(T, H)$. After Refs. [28, 260]

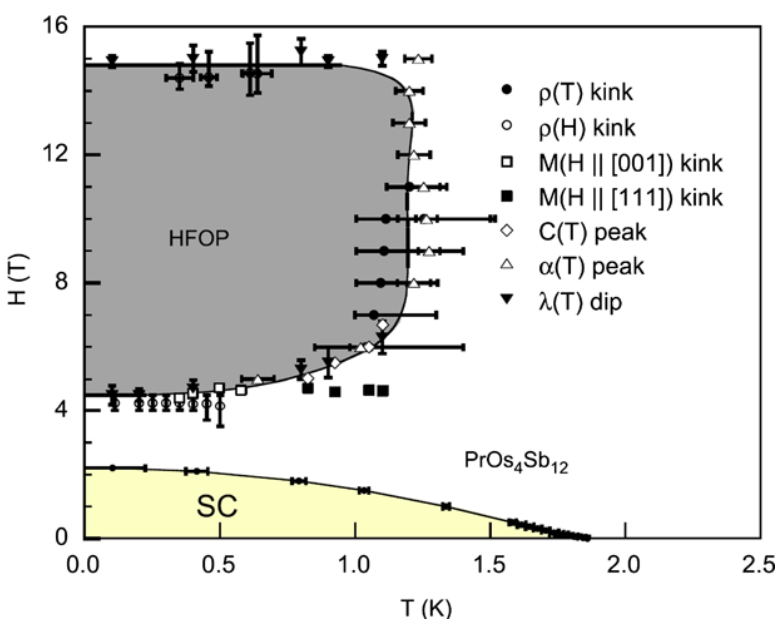

nomena [250]. Of these compounds, $\mathrm{PrOs}_{4} \mathrm{Sb}_{12}$ has received the most attention, owing largely to the fact that it is the first Pr-based heavy fermion superconductor, for which $T_{c}=1.85 \mathrm{~K}$ and $H_{c 2}(0)=2.5 \mathrm{~T}[27,28]$. The superconducting state breaks time reversal symmetry [252], apparently consists of several distinct superconducting phases, some of which may have point nodes in the energy gap [253, 254], and may involve triplet spin pairing of electrons [255]. Additionally, it has been shown that there likely are multiple superconducting bands [254, 256-259]. The superconductivity develops from a Fermi liquid ground state where the conduction electrons apparently have masses that are fifty times larger than the free electron mass [27, 28]. It is evident that the heavy quasiparticles participate in the superconductivity, in that the specific heat jump $\Delta_{C}$ is comparable with $\gamma T_{c}$ [28]. It has also been demonstrated that the superconductivity develops out of a non-magnetic $\Gamma_{1}$ crystalline electric field $(\mathrm{CEF})$ ground state with a low lying $\Gamma_{5}$ (using the notation for a cubic CEF) and that the application of magnetic fields larger than $4.5 \mathrm{~T}$ induces a high field ordered phase (HFOP) [28, 260] which is thought to be due to antiferroquadrupolar ordering [261] (Fig. 25). As a result of this wealth of unusual behaviors, $\mathrm{PrOs}_{4} \mathrm{Sb}_{12}$ has been the subject of much investigation since it's discovery in our lab in the early 2000s. However, in spite of the vigorous research that has been devoted to this compound, there yet remain several outstanding issues to be addressed.

The nature of the superconductivity in $\mathrm{PrOs}_{4} \mathrm{Sb}_{12}$ continues to be an unresolved issue. Experimental studies have reported various $H-T$ phase diagrams with mixed evidence for nodes in the pairing gap. Some experiments have also indicated the presence of multiple gaps, suggesting that the pairing involves either multiple bands or multiple order parameters. In order to address this issue, transverse field muon spin rotation (TF- $\mu \mathrm{SR})$ measurements, in fields up to $200 \mathrm{Oe}$, were performed. These measurements suggest that the superconducting penetration depth $\lambda(T)$ is temperature independent at low temperatures, consistent with a completely gapped quasiparticle excitation spectrum. In contrast, radio frequency (rf) inductive measurements yield a stronger temperature dependence of $\lambda(T)$, indicative of point nodes in the gap. It is possible that this discrepancy is related to multiband superconductivity [257, 
258]. It is also worth noting that measurements of the muon Knight-shift in the normal state of $\mathrm{PrOs}_{4} \mathrm{Sb}_{12}$ suggest that the perturbing effect of the muon charge on the neighboring $\operatorname{Pr}^{3+}$ crystalline electric field is negligibly small and therefore is unlikely to cause the difference between the TF- $\mu \mathrm{SR}$ and $\mathrm{rf}$ results. The superconducting state has also been probed via Andreev reflection spectroscopy measurements, revealing distinct spectral evidence for the presence of gap nodes, the evolution of which has been interpreted within a scenario for multigap pairing [262]. Thermal and charge conductivity measurements were recently performed for single crystal samples of $\mathrm{PrOs}_{4} \mathrm{Sb}_{12}$ and $\mathrm{PrRu}_{4} \mathrm{Sb}_{12}$. In zero magnetic field, the low temperature electronic thermal conductivity of $\mathrm{PrRu}_{4} \mathrm{Sb}_{12}$ is consistent with a fully gapped Fermi surface. For $\mathrm{PrOs}_{4} \mathrm{Sb}_{12}$, residual electronic conduction in the zero-temperature limit indicates the presence of nodes in the superconducting energy gap. The electronic thermal conductivity for both compounds shows a rapid rise at low magnetic fields. In $\mathrm{PrRu}_{4} \mathrm{Sb}_{12}$, this result is interpreted in terms of multiband effects. In $\mathrm{PrOs}_{4} \mathrm{Sb}_{12}$, the effect of the Doppler shift of nodal quasiparticles and multiband effects were considered [254, 259].

To further advance our understanding of $\mathrm{PrOs}_{4} \mathrm{Sb}_{12}$, studies of the substitutional systems $\operatorname{Pr}_{1-x} \mathrm{Nd}_{x} \mathrm{Os}_{4} \mathrm{Sb}_{12}$ and $\operatorname{Pr}\left(\mathrm{Os}_{1-x} \mathrm{Ru}_{x}\right)_{4} \mathrm{Sb}_{12}$ were undertaken. For the $\mathrm{Pr}_{1-x} \mathrm{Nd}_{x} \mathrm{Os}_{4} \mathrm{Sb}_{12}$ series, the $x=1$ end-member is a ferromagnet with an ordering temperature $T_{C} \sim 0.9 \mathrm{~K}$ [265]. As mentioned above, several measurements have indicated that the superconducting electron pairing in $\mathrm{PrOs}_{4} \mathrm{Sb}_{12}$ may be spin-triplet. This result implies that the superconductivity in $\mathrm{PrOs}_{4} \mathrm{Sb}_{12}$ could tolerate the presence of magnetic moments. In contrast, for the series $\operatorname{Pr}\left(\mathrm{Os}_{1-x} \mathrm{Ru}_{x}\right)_{4} \mathrm{Sb}_{12}$, the $x=1$ end-member is a conventional BCS superconductor [266]. Thus, it was expected that these substitutions would uniquely affect the unconventional superconductivity, heavy fermion behavior, and CEF effects, and thereby provide insight into the mechanisms underlying the unusual phenomena in the pure compound. The results of these studies are summarized in Fig. 26.

In order to determine the $T-x$ phase diagram for the $\operatorname{Pr}_{1-x} \mathrm{Nd}_{x} \mathrm{Os}_{4} \mathrm{Sb}_{12}$ system, measurements of ac magnetic susceptibility vs. temperature in zero-field and electrical resistivity vs. temperature in magnetic fields between $H=0 \mathrm{~T}$ and $8 \mathrm{~T}$ were performed for several concentrations $x$ [250, 267]. A phase diagram for $T_{c}$ and $T_{C}$ vs. $\mathrm{Nd}$ concentration was developed which reveals that superconductivity persists up to the surprisingly high value of $x \sim 0.5$ and ferromagnetism appears above $x \sim 0.5$. Both $T_{c}$ and $T_{C}$ are suppressed almost linearly toward $x=0.5$, at which point a sudden disappearance of the ferromagnetic feature in the ac magnetic susceptibility vs. temperature data suggests that this concentration may be near a possible quantum phase transition. The low temperature $H-x$ phase diagram was also developed, from which it is apparent that the normal state features related to the HFOP persist up to $x \sim 0.5$ (note that for $x>0.5$, measurements have yet to be performed). In contrast, while the HFOP is only weakly perturbed with increasing $x$ up to $0.5, H_{c 2}(T=0)$ is strongly suppressed up to $x \sim 0.3$, above which $H_{c 2}(T=0)$ decreases more gradually up to $x \sim 0.5$. These data, along with specific heat measurements indicate that the possible coexistence of ferromagnetism and superconductivity for $x>0.3$.

From measurements of electrical resistivity, dc magnetic susceptibility, ac magnetic susceptibility, and specific heat [28, 266, 268], the phase diagrams for 

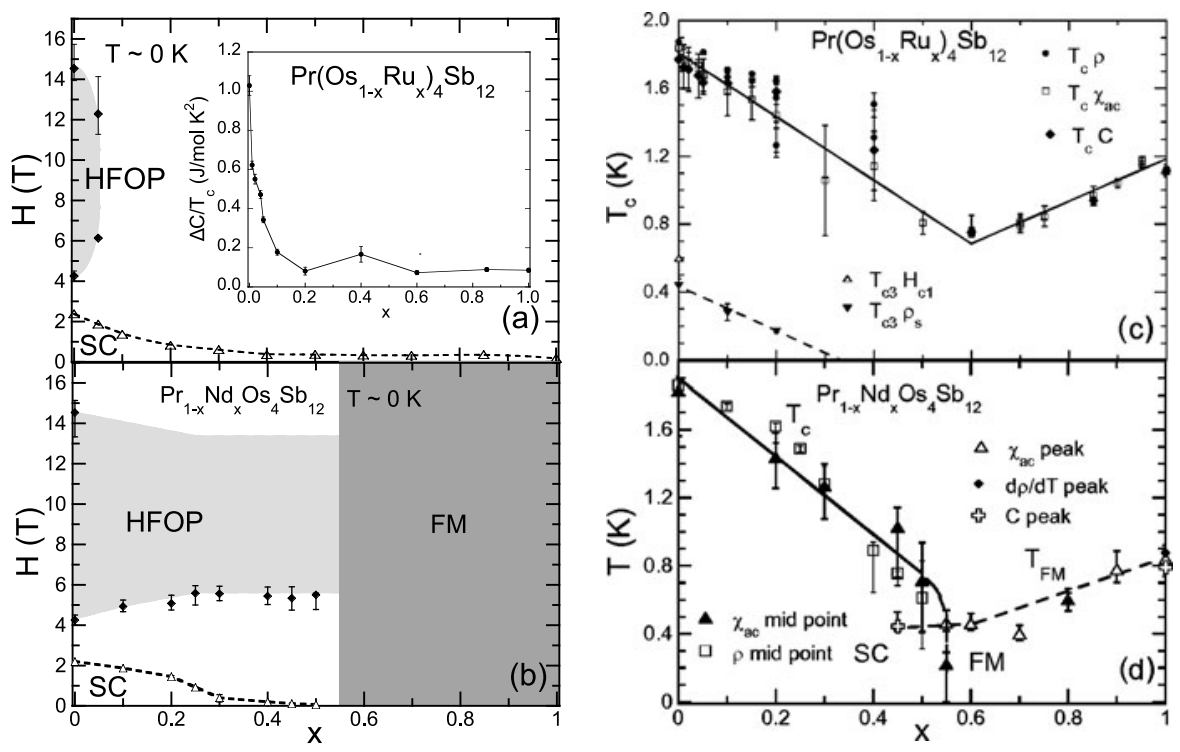

Fig. 26 Left panel (a) Zero-kelvin $H-x$ phase diagram for $\operatorname{Pr}\left(\mathrm{Os}_{1-x} \mathrm{Ru}_{x}\right)_{4} \mathrm{Sb}_{12}$. Left panel (b) Zero-kelvin $H-x$ phase diagram for $\operatorname{Pr}_{1-x} \mathrm{Nd}_{x} \mathrm{Os}_{4} \mathrm{Sb}_{12}$. The dashed line and the gray area are guides to the eye. Left panel (a) Inset: Ruthenium concentration $x$ dependence of the specific heat jump at the superconducting transition divided by the transition temperature $\Delta C / T_{c}$. Right panel (c) Superconducting transition temperature $T_{c}$ vs. Ru concentration $x$ in the $\operatorname{Pr}\left(\mathrm{Os}_{1-x} \mathrm{Ru}_{x}\right)_{4} \mathrm{Sb}_{12}$ system. The solid lines drawn from $x=0$ and $x=1$ toward $x=0.6$ are guides to the eye. The possible low temperature phase identified as $T_{c 3}$, which was determined from the $H_{c 1}(T)$ and magnetic penetration depth data [263, 264] is also plotted. Right panel (d) $T_{C}$ and the Curie transition temperature $T_{C}$ vs. Nd concentration $x$ in the $\mathrm{Pr}_{1-x} \mathrm{Nd}_{x} \mathrm{Os}_{4} \mathrm{Sb}_{12}$ system

$\operatorname{Pr}\left(\mathrm{Os}_{1-x} \mathrm{Ru}_{x}\right)_{4} \mathrm{Sb}_{12}$ were also established. As shown in Fig. 26 as $x$ increases, $T_{c}$ decreases to a minimum value at $x=0.6$, and then increases again to $x=1$. As noted earlier, it is also observed that the quantity $\Delta C / T_{c}$ is large for $x=0$, reflecting the large mass of the quasiparticles involved in the superconducting ground state. However, with increasing $x$, the large value for $\Delta C / T$ is suppressed to a constant value for $0.05<x$. Additionally, the double superconducting transition that is observed in specific heat measurements for the pure compound disappears for $0.05<x$ [269]. Thus, there apparently are two interesting Ru concentration regimes where the ground state may change: $x \sim 0.05$ and $x \sim 0.6$. In order to investigate this issue, the evolution of the superconductivity and the HFOP was measured by means of electrical resistivity in magnetic fields up to $18 \mathrm{~T}$. For $x<0.4$, the upper critical field curves are conventional. However, for $0.4<x$, the $H_{c 2}(T)$ curves are nearly linear [270]. This result may be consistent with the multi-band picture for superconductivity which has been proposed to explain features in several other measurements, as mentioned above. Interestingly, it was also revealed that the HFOP is weakened with increasing $x$ and suppressed by $x \sim 0.1$ [270], which is in the vicinity of the Ru concentration where many of the other features related to unconventional superconductivity are suppressed. 


\section{Concluding Remarks}

Over the years, NFL behavior has evolved from a rare curiosity to a common phenomenon. Although we have discussed several different materials in this review, they represent a small sample of NFL systems known today. In contrast to twenty years ago, the occurrence of NFL behavior is now observed in a wide variety of materials. Examples include materials with important applications potential, including the cuprate superconductors, and the recently discovered iron arsenide superconductors. Although NFL behavior is often associated with quantum critical fluctuations, it is evident there are many cases that do not fit this mold cleanly, including $\mathrm{Y}_{1-x} \mathrm{U}_{x} \mathrm{Pd}_{3}$, $\mathrm{URu}_{2-x} \mathrm{Re}_{x} \mathrm{Si}_{2}, \mathrm{Yb}_{2} \mathrm{Fe}_{12} \mathrm{P}_{7}, \mathrm{Ce}_{1-x} \mathrm{Y}_{x} \mathrm{CoIn}_{5}, \mathrm{MnSi}$, etc. It is also worth noting that there are many systems, such as $\mathrm{PrOs}_{4} \mathrm{Sb}_{12}$, which have interesting phase diagrams with evidence for a QCP, but do not exhibit NFL behavior. It is safe to say that today NFL behavior is not completely understood. Unraveling what these various materials share in common and what aspects of their NFL behavior are system-specific is a current very active field of research. Undoubtedly many more materials exhibiting NFL behavior will be discovered in the future.

Acknowledgements The crystal growth work was supported by the US Department of Energy (DOE) under research grant DE FG02-04ER46105 and acquisition of crystal growth equipment was sponsored by the US DOE through grant DE FG02-04ER46178. Low-temperature measurements were funded by the National Science Foundation (NSF) under grant 0802478. High-pressure research at University of California, San Diego (UCSD), was supported by the National Nuclear Security Administration (NNSA) under the Stewardship Science Academic Alliance (SSAA) program through the U.S. Department of Energy (DOE) grant number DEFG52-06NA26205. N. P. B. acknowledges support from CNAM. M.J. gratefully acknowledges financial support by the Alexander von Humboldt foundation.

Open Access This article is distributed under the terms of the Creative Commons Attribution Noncommercial License which permits any noncommercial use, distribution, and reproduction in any medium, provided the original author(s) and source are credited.

\section{References}

1. M.B. Maple, C.L. Seaman, D.A. Gajewski, Y. Dalichaouch, V.B. Barbetta, M.C. de Andrade, H.A. Mook, H.G. Lukefahr, O.O. Bernal, D.E. MacLaughlin, J. Low Temp. Phys. 95, 225 (1994)

2. M.B. Maple, M.C. de Andrade, J. Herrmann, Y. Dalichaouch, D.A. Gajewski, C.L. Seaman, R. Chau, R. Movshovich, M.C. Aronson, R. Osborn, J. Low Temp. Phys. 99, 223 (1995)

3. P. Coleman, M.B. Maple, A.J. Millis (eds.), J. Phys. Condens. Matt. 8 (See various articles in Proceedings of the Institute for Theoretical Physics Conference on Non-Fermi Liquid Behavior in Metals, Santa Barbara) (1996)

4. G.R. Stewart, Rev. Mod. Phys. 73, 797 (2001)

5. G.R. Stewart, Rev. Mod. Phys. 78, 743 (2006)

6. C.L. Seaman, M.B. Maple, B.W. Lee, S. Ghamaty, M.S. Torikachvili, J.-S. Kang, L.Z. Liu, J.W. Allen, D.L. Cox, Phys. Rev. Lett. 67, 2882 (1991)

7. H. v. Löhneysen, J. Phys., Condens. Matter 8, 9689 (1996)

8. F. Steglich, B. Buschinger, P. Gegenwart, M. Lohmann, R. Helfrich, C. Langhammer, P. Hellmann, L. Donnevert, S. Thomas, A. Link, C. Geibel, M. Lang, G. Sparn, W. Assmus, J. Phys., Condens. Matter 8, 9909 (1996)

9. S.R. Julian, F.V. Carter, F.M. Grosche, R.K.W. Haselwimmer, S.J. Lister, N.D. Mathur, G.J. McMullan, C. Pfleiderer, S.S. Saxena, I.R. Walker, N.J.W. Wilson, G.G. Lonzarich, J. Magn. Magn. Mater. 177-181, 265 (1998) 
10. O.O. Bernal, D.E. MacLaughlin, H.G. Lukefahr, B. Andraka, Phy. Rev. Lett. 75, 2023 (1995)

11. E. Miranda, V. Dobrosavljević, G. Kotliar, J. Phys., Condens. Matter 8, 9871 (1996)

12. P. Nozières, A. Blandin, J. Phys. (Paris) 41, 193 (1980)

13. A.M. Tsvelik, J. Phys. C 18, 159 (1985)

14. D.L. Cox, Phys. Rev. Lett. 59, 1240 (1987)

15. A.W.W. Ludwig, I. Affleck, Phys. Rev. Lett. 67, 3160 (1991)

16. P. Schlottmann, P.D. Sacramento, Adv. Phys. 42, 641 (1993)

17. A.H. Castro Neto, G. Castilla, B.A. Jones, Phys. Rev. Lett. 81, 3531 (1998)

18. N.D. Mathur, F.M. Grosche, S.R. Julian, I.R. Walker, D.M. Freye, R.K.W. Haselwimmer, G.G. Lonzarich, Nature 394, 39 (1998)

19. H. Hegger, C. Petrovic, E.G. Moshopoulou, M.F. Hundley, J.L. Sarrao, Z. Fisk, J.D. Thompson, Phys. Rev. Lett. 84, 4986 (2000)

20. S.S. Saxena, P.B. Littlewood, Nature 412, 290 (2001)

21. E.D. Bauer, R.P. Dickey, V.S. Zapf, M.B. Maple, J. Phys., Condens. Matter 13, L759 (2001)

22. F. Hardy, A.D. Huxley, Phys. Rev. Lett. 94, 247006 (2005)

23. F. Lévy, I. Sheikin, B. Grenier, A.D. Huxley, Science 309, 1343 (2005)

24. D. Aoki, A.D. Huxley, E. Ressouche, D. Braithwaite, J. Flouquet, J.P. Brison, E. Lhotel, C. Paulsen, Nature (London) 413, 613 (2001)

25. E. Hassinger, D. Aoki, G. Knebel, J. Flouquet, J. Phys. Soc. Jpn. 77(7), 073703 (2008)

26. E. Slooten, T. Naka, A. Gasparini, Y.K. Huang, A. de Visser, Phys. Rev. Lett. 103, 097003 (2009)

27. E.D. Bauer, N.A. Frederick, P.-C. Ho, V.S. Zapf, M.B. Maple, Phys. Rev. B 65, 100506 (2002)

28. M.B. Maple, N.A. Frederick, P.-C. Ho, W.M. Yuhasz, T. Yanagisawa, J. Supercond. Novel Magn. 19, 299 (2006)

29. C. Pfleiderer, P. Böni, T. Keller, U.K. Rößler, A. Rosch, Science 316, 1871 (2007)

30. D. Jaccard, A.T. Holmes, G. Behr, Y. Inada, Y. Onuki, Phys. Lett. A 299, 282 (2002)

31. A.T. Holmes, D. Jaccard, G. Behr, Y. Inada, Y. Onuki, J. Phys., Condens. Matter 16, S1121 (2004)

32. D.L. Cox, M.B. Maple, Phys. Today 48, 32 (1995)

33. R.P. Dickey, V.S. Zapf, P.-C. Ho, E.J. Freeman, N.A. Frederick, M.B. Maple, Phys. Rev. B 68, 104404 (2003)

34. J. Paglione, T.A. Sayles, P.C. Ho, J.R. Jeffries, M.B. Maple, Nat. Phys. 3, 703 (2007)

35. M.C. de Andrade, R. Chau, R.P. Dickey, N.R. Dilley, E.J. Freeman, D.A. Gajewski, M.B. Maple, R. Movshovich, A.H.C. Neto, G. Castilla, B.A. Jones, Phys. Rev. Lett. 81, 5620 (1998)

36. H.G. Lukefahr, O.O. Bernal, D.E. MacLaughlin, C.L. Seaman, M.B. Maple, B. Andraka, Phys. Rev. B 52, 3038 (1995)

37. T. Moriya, Spin Fluctuations in Itinerant Electron Magnetism (Springer, Berlin, 1985)

38. T. Moriya, T. Takimoto, J. Phys. Soc. Jpn. 64, 960 (1995)

39. B. Andraka, A.M. Tsvelik, Phys. Rev. Lett. 67, 2886 (1991)

40. J.A. Hertz, Phys. Rev. B 14(3), 1165 (1976)

41. A.J. Millis, Phys. Rev. B 48, 7183 (1993)

42. M.A. Continentino, Phys. Rev. B 47, 11587 (1993)

43. A.M. Tsvelik, M. Reizer, Phys. Rev. B 48, 9887 (1993)

44. S. Sachdev, N. Read, R. Oppermann, Phys. Rev. B 52, 10286 (1995)

45. S.J. Yamamoto, Q. Si, Proc. Natl. Acad. Sci. USA (2010, unpublished)

46. H. v. Löhneysen, T. Pietrus, G. Portisch, H.G. Schlager, A. Schröder, M. Sieck, T. Trappman, Phys. Rev. Lett. 472, 3262 (1994)

47. O. Trovarelli, C. Geibel, S. Mederle, C. Langhammer, F.M. Grosche, P. Gegenwart, M. Lang, G. Sparn, F. Steglich, Phys. Rev. Lett. 85, 626 (2000)

48. C.L. Seaman, M.B. Maple, B.W. Lee, S. Ghamaty, M.S. Torikachvili, J.S. Kang, L.Z. Liu, J.W. Allen, D.L. Cox, J. Alloys Compd. 181, 327 (1992)

49. M.B. Maple, R.P. Dickey, J. Herrmann, M.C. de Andrade, E.J. Freeman, D.A. Gajewski, R. Chau, J. Phys., Condens. Matter 8, 9773 (1996)

50. C.L. Seaman, M.B. Maple, Physica B 199-200, 396 (1994)

51. J.-S. Kang, J.W. Allen, M.B. Maple, M.S. Torikachvili, W.P. Ellis, B.B. Pate, Z.X. Shen, J.J. Yeh, I. Lindau, Phys. Rev. B 39, 13529 (1989)

52. D.A. Gajewski, R. Chau, M.B. Maple, Phys. Rev. B 62, 5496 (2000)

53. P.D. Sacramento, P. Schlottmann, Phys. Lett. A 142, 245 (1989)

54. D.L. Cox, M. Makivic, Physica B 199-200, 391 (1994)

55. H.A. Mook, C.L. Seaman, M.B. Maple, M.A.L. de la Torre, D.L. Cox, M. Makivic, Physica B 186188, 341 (1993) 
56. P. Dai, H.A. Mook, C.L. Seaman, M.B. Maple, J.P. Koster, Phys. Rev. Lett. 75, 1202 (1995)

57. M.J. Bull, K.A. McEwen, R.S. Eccleston, Phys. Rev. B 57, 3850 (1998)

58. D.A. Gajewski, P. Allenspach, C.L. Seaman, M.B. Maple, Physica B 199-200, 419 (1994)

59. S. Süllow, T.J. Gortenmulder, G.J. Nieuwenhuys, A.A. Menovsky, J.A. Mydosh, J. Alloys Compd. 215, 223 (1994)

60. J. Xu, P.J.C. Signore, B. Andraka, W.A. Acree, M.W. Meisel, Y. Takano, J. Alloys Compd. 216, 33 (1994)

61. S.D. Wilson, P.C. Dai, D.T. Adroja, S.-H. Lee, J.-H. Chung, J.W. Lynn, N.P. Butch, M.B. Maple, Phys. Rev. Lett. 94, 056402 (2005)

62. M.C. Aronson, R. Osborn, R.A. Robinson, J.W. Lynn, R. Chau, C.L. Seaman, M.B. Maple, Phys. Rev. Lett. 75, 725 (1995)

63. M.C. Aronson, R. Osborn, R. Chau, M.B. Maple, B.D. Rainford, A.P. Murani, Phys. Rev. Lett. 87, 197205 (2001)

64. A. Schröder, G. Aeppli, E. Bucher, R. Ramazashvili, P. Coleman, Phys. Rev. Lett. 80, 5623 (1998)

65. N.P. Butch, M.B. Maple, Phys. Rev. Lett. 103, 076404 (2009)

66. C. Geibel, C. Schank, S. Thies, H. Kitazawa, C. Bredl, A. Böhm, M. Rau, A. Grauel, R. Caspary, R. Helfrich, U. Ahlheim, G. Weber, F. Steglich, Z. Phys. B 84, 1 (1991)

67. A. Krimmel, P. Fischer, B. Roessli, H. Maletta, C. Geibel, C. Schank, A. Grauel, A. Loidl, F. Steglich, Z. Phys. B 86, 161 (1992)

68. C. Geibel, C. Schank, F. Jährling, B. Buschinger, A. Grauel, T. Lühman, P. Gegenwart, R. Helfrich, P. Reinders, F. Steglich, Physica B 199-200, 128 (1994)

69. Y. Dalichaouch, M.B. Maple, Physica B 199-200, 176 (1994)

70. R.P. Dickey, A. Amann, E.J. Freeman, M.C. de Andrade, M.B. Maple, Phys. Rev. B 62, 3979 (2000)

71. A.M. Strydom, P.V. de du Plessis, R. Troc, Physica B 259-261, 421 (1999)

72. E.J. Freeman, M.C. de Andrade, R.P. Dickey, N.R. Dilley, M.B. Maple, Phys. Rev. B 58, 16027 (1998)

73. E.J. Freeman, M.B. Maple (unpublished)

74. V.S. Zapf, E.J. Freeman, R.P. Dickey, P.-C. Ho, M.B. Maple, Physica B 312-313, 448 (2002)

75. M.B. Maple, E.D. Bauer, V.S. Zapf, J. Wosnitza, Superconductivity in Conventional and Unconventional Superconductors (Springer, Berlin, 2008), pp. 639-762

76. V.S. Zapf, R.P. Dickey, E.J. Freeman, C. Sirvent, M.B. Maple, Phys. Rev. B 65, 024437 (2001)

77. M.B. Maple, J. Chen, Y. Dalichaouch, T. Kohara, C. Rossel, M.S. Torikachvili, M.W. McElfresh, J.D. Thompson, Phys. Rev. Lett. 56, 185 (1986)

78. T.T.M. Palstra, A.A. Menovsky, J. van den Berg, A.J. Dirkmaat, P.H. Kes, G.J. Nieuwenhuys, J.A. Mydosh, Phys. Rev. Lett. 55, 2727 (1985)

79. W. Schlabitz, J. Baumann, B. Pollit, U. Rauchschwalbe, H.M. Mayer, U. Ahlheim, C.D. Bredl, Z. Phys. B 62, 171 (1986)

80. C. Broholm, J.K. Kjems, W.J.L. Buyers, P. Matthews, T.T.M. Palstra, A.A. Menovsky, J.A. Mydosh, Phys. Rev. Lett. 58, 1467 (1987)

81. H. Amitsuka, K. Matsuda, I. Kawasaki, K. Tenya, M. Yokoyama, C. Sekine, N. Tateiwa, T.C. Kobayashi, S. Kawarazaki, H. Yoshizawa, J. Magn. Magn. Mater. 310, 214 (2007)

82. H. Harima, K. Miyake, J. Flouquet, J. Phys. Soc. Jpn. 79, 033705 (2010)

83. S. Elgazzar, J. Rusz, M. Amft, P.M. Oppeneer, J.A. Mydosh, Nat. Mater. 8, 337 (2009)

84. A.V. Balatsky, A. Chantis, H.P. Dahal, D. Parker, J.X. Zhu, Phys. Rev. B 79, 214413 (2009)

85. K. Haule, G. Kotliar, Nat. Phys. 5, 796 (2009)

86. K. Kuwahara, H. Amitsuka, T. Sakakibara, O. Suzuki, S. Nakamura, T. Goto, M. Mihalik, A.A. Menovsky, A. de Visser, J.J.M. Franse, J. Phys. Soc. Jpn. 66, 3251 (1997)

87. A. de Visser, F.E. Kayzel, A.A. Menovksy, J.J.M. Franse, J. van den Berg, G.J. Nieuwenhuys, Phys. Rev. B 34, 8168 (1986)

88. P.A. Sharma, N. Harrison, M. Jaime, Y.S. Oh, K.H. Kim, C.D. Batista, H. Amitsuka, J.A. Mydosh, Phys. Rev. Lett. 97, 156401 (2006)

89. K. Hasselbach, J.R. Kirtley, P. Lejay, Phys. Rev. B 46, 5826 (1992)

90. C.R. Wiebe, J.A. Janik, G.J. MacDougall, G.M. Luke, J.D. Garrett, H.D. Zhou, Y.-J. Jo, L. Balicas, Y. Qiu, J.R.D. Copley, Z. Yamani, W.J.L. Buyers, Nat. Phys. 3, 96 (2007)

91. A.F. Santander-Syro, M. Klein, F.L. Boariu, A. Nuber, P. Lejay, F. Reinert, Nat. Phys. 5, 637 (2009)

92. M.W. McElfresh, J.D. Thompson, J.O. Willis, M.B. Maple, T. Kohara, M.S. Torikachvili, Phys. Rev. B 35, 43 (1987)

93. J.R. Jeffries, N.P. Butch, B.T. Yukich, M.B. Maple, Phys. Rev. Lett. 99, 217207 (2007) 
94. Y. Kasahara, T. Iwasawa, H. Shishido, T. Shibauchi, K. Behnia, Y. Haga, T.D. Matsuda, Y. Onuki, M. Sigrist, Y. Matsuda, Phys. Rev. Lett. 99, 116402 (2007)

95. E. Hassinger, G. Knebel, K. Izawa, P. Lejay, B. Salce, J. Flouquet, Phys. Rev. B 77, 115117 (2008)

96. N.P. Butch, J.R. Jeffries, S.X. Chi, J.B. Leão, J.W. Lynn, M.B. Maple, arXiv:1006.4140 (2010, unpublished)

97. J.R. Jeffries, N.P. Butch, B.T. Yukich, M.B. Maple, J. Phys., Condens. Matter 20, 095225 (2008)

98. N.P. Butch, J.R. Jeffries, D.A. Zocco, M.B. Maple, High Press. Res. 29, 335 (2009)

99. C. Pfleiderer, J.A. Mydosh, M. Vojta, Phys. Rev. B 74, 104412 (2006)

100. R.A. Fisher, S. Kim, Y. Wu, N.E. Phillips, M.W. McElfresh, M.S. Torikachvili, M.B. Maple, Physica B 163, 419 (1990)

101. F.R. de Boer, J.J.M. Franse, E. Louis, A.A. Menovsky, J.A. Mydosh, T.T.M. Palstra, U. Rauchschwalbe, W. Schlabitz, F. Steglich, A. de Visser, Physica B+C 138, 1 (1986)

102. A. de Visser, F. de Boer, A.A. Menovsky, J.J.M. Franse, Sol. State Commun. 64, 527 (1987)

103. M. Jaime, K.H. Kim, G. Jorge, S. McCall, J.A. Mydosh, Phys. Rev. Lett. 89, 287201 (2002)

104. N. Harrison, M. Jaime, J.A. Mydosh, Phys. Rev. Lett. 90, 096402 (2003)

105. K.H. Kim, N. Harrison, M. Jaime, G.S. Boebinger, J.A. Mydosh, Phys. Rev. Lett. 91, 256401 (2003)

106. J.S. Kim, D. Hall, P. Kumar, G.R. Stewart, Phys. Rev. B 67, 014404 (2003)

107. Y.S. Oh, K.H. Kim, P.A. Sharma, N. Harrison, H. Amitsuka, J.A. Mydosh, Phys. Rev. Lett. 98, 016401 (2007)

108. K.H. Kim, N. Harrison, H. Amitsuka, G.A. Jorge, M. Jaime, J.A. Mydosh, Phys. Rev. Lett. 93, 206402 (2004)

109. A.V. Silhanek, N. Harrison, C.D. Batista, M. Jaime, A. Lacerda, H. Amitsuka, J.A. Mydosh, Phys. Rev. Lett. 95, 026403 (2005)

110. Z. Zhu, E. Hassinger, Z. Xu, D. Aoki, J. Flouquet, K. Behnia, Phys. Rev. B 80, 172501 (2009)

111. Y. Dalichaouch, M.B. Maple, M.S. Torikachvili, A.L. Giorgi, Phys. Rev. B 39, 2423 (1989)

112. Y. Dalichaouch, M.B. Maple, J.W. Chen, T. Kohara, C. Rossel, M.S. Torikachvili, A.L. Giorgi, Phys. Rev. B 41, 1829 (1990)

113. Y. Miyako, S. Kawarazaki, H. Amitsuka, C.C. Paulsen, K. Hasselbach, J. Appl. Phys. 70, 5791 (1991)

114. Y. Dalichaouch, M.B. Maple, R.P. Guertin, M.V. Kuric, M.S. Torikachvili, A.L. Giorgi, Physica B 163, 113 (1990)

115. N.P. Butch, M.B. Maple, J. Phys., Condens. Matter 22, 164204 (2010)

116. M.S. Torikachvili, L. Rebelsky, K. Motoya, S.M. Shapiro, Y. Dalichaouch, M.B. Maple, Phys. Rev. B 45, 2262 (1992)

117. Y. Kohori, Y. Noguchi, T. Kohara, Y. Dalichaouch, M.A. Lopez de la Torre, M.B. Maple, Physica B 186, 792 (1993)

118. S. Thieme, P. Steiner, L. Degiorgi, P. Wachter, Y. Dalichaouch, M.B. Maple, Europhys. Lett. 32, 367 (1995)

119. E.D. Bauer, V.S. Zapf, P.-C. Ho, N.P. Butch, E.J. Freeman, C. Sirvent, M.B. Maple, Phys. Rev. Lett. 94, 046401 (2005)

120. V.V. Krishnamurthy, D.T. Adroja, N.P. Butch, S.K. Sinha, M.B. Maple, R. Osborn, J.L. Robertson, S.E. Nagler, M.C. Aronson, Phys. Rev. B 78, 024413 (2008)

121. N.P. Butch, Ph.D. dissertation, University of California, San Diego (2008)

122. J.D. Thompson, R. Movshovich, Z. Fisk, F. Bouquet, N.J. Curro, R.A. Fisher, P.C. Hammel, H. Hegger, M.F. Hundley, M. Jaime, P.G. Pagliuso, C. Petrovic, N.E. Phillips, J.L. Sarrao, J. Magn. Magn. Mater. 226-230, 5 (2001)

123. P. Morin, C. Vettier, J. Flouquet, M. Konczykowski, Y. Lassailly, J.M. Mignot, U. Welp, J. Low Temp. Phys. 70, 377 (1988)

124. I.R. Walker, F.M. Grosche, D.M. Freye, G.G. Lonzarich, Physica C 282-287, 303 (1997)

125. G. Knebel, D. Braithwaite, P.C. Canfield, G. Lapertot, J. Flouquet, Phys. Rev. B 65, 024425 (2001)

126. R. Settai, T. Kubo, T.D. Matsuda, Y. Haga, Y. Ōnuki, H. Harima, J. Phys. Soc. Jpn. 75S, 167 (2005)

127. S. Doniach, Physica B 91, 231 (1977)

128. S. Kawasaki, M. Yashima, Y. Kitaoka, K. Takeda, K. Shimizu, Y. Oishi, M. Takata, T.C. Kobayashi, H. Harima, S. Araki, H. Shishido, R. Settai, Y. Ōnuki, Phys. Rev. B 77, 064508 (2008)

129. W. Bao, P.G. Pagliuso, J.L. Sarrao, J.D. Thompson, Z. Fisk, J.W. Lynn, R.W. Erwin, Phys. Rev. B 62, R14621 (2000)

130. C. Petrovic, R. Movshovich, M. Jaime, P.G. Pagliuso, M.F. Hundley, J.L. Sarrao, Z. Fisk, J.D. Thompson, Europhys. Lett. 53, 354 (2001) 
131. C. Petrovic, P.G. Pagliuso, M.F. Hundley, R. Movshovich, J.L. Sarrao, J.D. Thompson, Z. Fisk, P. Monthoux, J. Phys., Condens. Matter 13, L337 (2001)

132. T. Muramatsu, Physica C 388-389, 539 (2003)

133. J. Paglione, P.C. Ho, M.B. Maple, M.A. Tanatar, L. Taillefer, Y. Lee, C. Petrovic, Phys. Rev. B 77, 100505 (2008)

134. P. Monthoux, G.G. Lonzarich, Phys. Rev. B 66, 224504 (2002)

135. R. Movshovich, M. Jaime, J.D. Thompson, C. Petrovic, Z. Fisk, P.G. Pagliuso, J.L. Sarrao, Phys. Rev. Lett. 86, 5152 (2001)

136. G.Q. Zheng, K. Tanabe, T. Mito, S. Kawasaki, Y. Kitaoka, D. Aoki, Y. Haga, Y. Onuki, Phys. Rev. Lett. 86, 4664 (2001)

137. Y. Kohori, Y. Yamato, Y. Iwamoto, T. Kohara, E.D. Bauer, M.B. Maple, J.L. Sarrao, Phys. Rev. B 64, 134526 (2001)

138. T. Mito, S. Kawasaki, G.Q. Zheng, Y. Kawasaki, K. Ishida, Y. Kitaoka, D. Aoki, Y. Haga, Y. Onuki, Phys. Rev. B 63, 220507 (2001)

139. A. Bianchi, R. Movshovich, N. Oeschler, P. Gegenwart, F. Steglich, J.D. Thompson, P.G. Pagliuso, J.L. Sarrao, Phys. Rev. Lett. 89, 137002 (2002)

140. R.J. Ormeno, A. Sibley, C.E. Gough, S. Sebastian, I.R. Fisher, Phys. Rev. Lett. 88, 047005 (2002)

141. Y. Kasahara, Y. Nakajima, K. Izawa, Y. Matsuda, K. Behnia, H. Shishido, R. Settai, Y. Onuki, Phys. Rev. B 72, 214515 (2005)

142. P. Fulde, R.A. Ferrell, Phys. Rev. 135, A550 (1964)

143. A.I. Larkin, Y.N. Ovchinnikov, Sov. Phys. JETP 20, 762 (1965)

144. A. Bianchi, R. Movshovich, C. Capan, P.G. Pagliuso, J.L. Sarrao, Phys. Rev. Lett. 91, 187004 (2003)

145. H.A. Radovan, N.A. Fortune, T.P. Murphy, S.T. Hannahs, E.C. Palm, S.W. Tozer, D. Hall, Nature 425, 51 (2003)

146. K. Kakuyanagi, M. Saitoh, K. Kumagai, S. Takashima, M. Nohara, H. Takagi, Y. Matsuda, Phys. Rev. Lett. 94, 047602 (2005)

147. K. Kumagai, M. Saitoh, T. Oyaizu, Y. Furukawa, S. Takashima, M. Nohara, H. Takagi, Y. Matsuda, Phys. Rev. Lett. 97, 227002 (2006)

148. V.F. Mitrović, G. Koutroulakis, M. Klanjšek, M. Horvatić, C. Berthier, G. Knebel, G. Lapertot, J. Flouquet, Phys. Rev. Lett. 101, 039701 (2008)

149. B.L. Young, R.R. Urbano, N.J. Curro, J.D. Thompson, J.L. Sarrao, A.B. Vorontsov, M.J. Graf, Phys. Rev. Lett. 98, 036402 (2007)

150. M. Kenzelmann, T. Strassle, C. Niedermayer, M. Sigrist, B. Padmanabhan, M. Zolliker, A.D. Bianchi, R. Movshovich, E.D. Bauer, J.L. Sarrao, J.D. Thompson, Science 321, 1652 (2008)

151. A. Aperis, G. Varelogiannis, P.B. Littlewood, Phys. Rev. Lett. 104, 216403 (2010)

152. J.D. Thompson, M. Nicklas, A. Bianchi, R. Movshovich, A. Llobet, W. Bao, A. Malinowski, M.F. Hundley, N.O. Moreno, P.G. Pagliuso, J.L. Sarrao, S. Nakatsuji, Z. Fisk, R. Borth, E. Lengyel, N. Oeschler, G. Sparn, F. Steglich, Physica B 329-333, 446 (2003)

153. J.S. Kim, J. Alwood, G.R. Stewart, J.L. Sarrao, J.D. Thompson, Phys. Rev. B 64, 134524 (2001)

154. V.A. Sidorov, M. Nicklas, P.G. Pagliuso, J.L. Sarrao, Y. Bang, A.V. Balatsky, J.D. Thompson, Phys. Rev. Lett. 89, 157004 (2002)

155. J. Paglione, M.A. Tanatar, D.G. Hawthorn, E. Boaknin, R.W. Hill, F. Ronning, M. Sutherland, L. Taillefer, C. Petrovic, P.C. Canfield, Phys. Rev. Lett. 91, 246405 (2003)

156. A. Bianchi, R. Movshovich, I. Vekhter, P.G. Pagliuso, J.L. Sarrao, Phys. Rev. Lett. 91, 257001 (2003)

157. J.L. Sarrao, J.D. Thompson, J. Phys. Soc. Jpn. 76, 051013 (2007)

158. V.S. Zapf, E.J. Freeman, E.D. Bauer, J. Petricka, C. Sirvent, N.A. Frederick, R.P. Dickey, M.B. Maple, Phys. Rev. B 65, 014506 (2001)

159. J.R. Jeffries, N.A. Frederick, E.D. Bauer, H. Kimura, V.S. Zapf, K.D. Hof, T.A. Sayles, M.B. Maple, Phys. Rev. B 72, 024551 (2005)

160. M. Nicklas, V.A. Sidorov, H.A. Borges, P.G. Pagliuso, J.L. Sarrao, J.D. Thompson, Phys. Rev. B 70, 020505 (2004)

161. V.S. Zapf, N.A. Frederick, K.L. Rogers, K.D. Hof, P.C. Ho, E.D. Bauer, M.B. Maple, Phys. Rev. B 67, 064405 (2003)

162. P. Bak, M.H. Jensen, J. Phys. C 13, L881 (1980)

163. G. Shirane, R. Cowley, C. Majkrzak, J. Sokoloff, B. Pagonis, C.H. Perry, Y. Ishikawa, Phys. Rev. B 28, 6251 (1983)

164. M. Ishida, Y. Endoh, S. Mitsuda, Y. Ishikawa, M. Tanaka, J. Phys. Soc. Jpn. 54, 2975 (1985) 
165. I.E. Dzyaloshinskii, J. Phys. Chem. Solids 4, 241 (1958)

166. T. Moriya, Phys. Rev. 120, 91 (1960)

167. L. Taillefer, G. Lonzarich, P. Strange, J. Magn. Magn. Mater. 54-57, 957 (1986)

168. J. Thompson, Z. Fisk, G.G. Lonzarich, Physica B 161, 317 (1989)

169. C. Pfleiderer, R. Friend, G. Lonzarich, N. Bernhoeft, J. Flouquet, Int. J. Mod. Phys. B 7, 887 (1993)

170. C. Pfleiderer, G. McMullan, G. Lonzarich, Physica B 199, 634 (1994)

171. C. Pfleiderer, S.R. Julian, G.G. Lonzarich, Nature 414, 427 (2001)

172. N. Doiron-Leyraud, I.R. Walker, L. Taillefer, M.J. Steiner, S.R. Julian, G.G. Lonzarich, Nature 425, 595 (2003)

173. C. Pfleiderer, Physica B 328, 100 (2003)

174. P. Pedrazzini, D. Jaccard, G. Lapertot, J. Flouquet, Y. Inada, H. Kohara, Y. Onuki, Physica B 378, 165 (2006)

175. D. Belitz, T.R. Kirkpatrick, A. Rosch, Phys. Rev. B 73, 054431 (2006)

176. M. Janoschek, F. Bernlochner, S. Dunsiger, C. Pfleiderer, P. Böni, B. Roessli, P. Link, A. Rosch, Phys. Rev. B 81, 214436 (2010)

177. G.G. Lonzarich, L. Taillefer, J. Phys. C 18, 4339 (1985)

178. C. Thessieu, C. Pfleiderer, A.N. Stepanov, J. Flouquet, J. Phys., Condens. Matter 9, 6677 (1997)

179. C. Pfleiderer, G.J. McMullan, S.R. Julian, G.G. Lonzarich, Phys. Rev. B 55, 8330 (1997)

180. C. Pfleiderer, D. Reznik, L. Pintschovius, H. v. Löhneysen, M. Garst, A. Rosch, Nature 427, 227 (2004)

181. Y.J. Uemura, T. Goko, I.M. Gat-Malureanu, J.P. Carlo, P.L. Russo, A.T. Savici, A. Aczel, G.J. MacDougall, J.A. Rodriguez, G.M. Luke, S.R. Dunsiger, A. McCollam, J. Arai, C. Pfleiderer, P. Böni, K. Yoshimura, E. Baggio-Saitovitch, M.B. Fontes, J. Larrea, Y.V. Sushko, J. Sereni, Nat. Phys. 3, 29 (2007)

182. W. Yu, F. Zamborszky, J.D. Thompson, J.L. Sarrao, M.E. Torelli, Z. Fisk, S.E. Brown, Phys. Rev. Lett. 92, 086403 (2004)

183. M. Garst, A. Rosch, Phys. Rev. B 72, 205129 (2005)

184. T.R. Kirkpatrick, D. Belitz, R. Saha, Phys. Rev. B 78, 094407 (2008)

185. U.K. Rößler, A.N. Bogdanov, C. Pfleiderer, Nature 442, 797 (2006)

186. B. Binz, A. Vishwanath, V. Aji, Phys. Rev. Lett. 96, 207202 (2006)

187. S. Tewari, D. Belitz, T.R. Kirkpatrick, Phys. Rev. Lett. 96, 047207 (2006)

188. I. Fischer, N. Shah, A. Rosch, Phys. Rev. B 77, 024415 (2008)

189. S. Mühlbauer, B. Binz, F. Jonietz, C. Pfleiderer, A. Rosch, A. Neubauer, R. Georgii, P. Böni, Science 323, 915 (2009)

190. A. Neubauer, C. Pfleiderer, B. Binz, A. Rosch, R. Ritz, P.G. Niklowitz, P. Böni, Phys. Rev. Lett. 102, $186602(2009)$

191. M. Janoschek, F. Jonietz, P. Link, C. Pfleiderer, P. Böni, J. Phys., Conf. Ser. 200, 032026 (2010)

192. R. Georgii, P. Böni, D. Lamago, S. Stüber, S.V. Grigoriev, S.V. Maleyev, A.I. Okorokov, H. Eckerlebe, P.K. Pranzas, B. Roessli, W.E. Fischer, Physica B 350, 45 (2004)

193. S.V. Grigoriev, S.V. Maleyev, A.I. Okorokov, Y.O. Chetverikov, R. Georgii, P. Böni, D. Lamago, H. Eckerlebe, K. Pranzas, Phys. Rev. B 72, 134420 (2005)

194. M. Matsunaga, Y. Ishikawa, T. Nakajima, J. Phys. Soc. Jpn. 51, 1153 (1982)

195. C. Pfleiderer, J. Magn. Magn. Mater. 226-230, 23, (2001)

196. S.M. Stishov, A.E. Petrova, S. Khasanov, G.K. Panova, A.A. Shikov, J.C. Lashley, D. Wu, T.A. Lograsso, Phys. Rev. B 76, 052405 (2007)

197. A.E. Petrova, S.M. Stishov, J. Phys., Condens. Matter 21, 196001 (2009)

198. S.V. Grigoriev, S.V. Maleyev, E.V. Moskvin, V.A. Dyadkin, P. Fouquet, H. Eckerlebe, Phys. Rev. B 81, 144413 (2010)

199. C. Pappas, E. Lelièvre-Berna, P. Falus, P.M. Bentley, E. Moskvin, S. Grigoriev, P. Fouquet, B. Farago, Phys. Rev. Lett. 102, 197202 (2009)

200. W.A. Fertig, D.C. Johnston, E. DeLong, R.W. McCallum, M.B. Maple, M.T. Matthias, Phys. Rev. Lett. 38, 987 (1977)

201. D.E. Moncton, D.B. McWhan, J. Eckert, G. Shirane, W. Thomlinson, Phys. Rev. Lett. 39, 1164 (1977)

202. D.E. Moncton, D.B. McWhan, P.H. Schmidt, G. Shirane, W. Thomlinson, M.B. Maple, H.B. MacKay, L.D. Woolf, Z. Fisk, D.C. Johnston, Phys. Rev. Lett. 45, 2060 (2008)

203. S.K. Sinha, G.W. Crabtree, D.G. Hinks, H. Mook, Phys. Rev. Lett. 48, 950 (1982)

204. M. Ishikawa, Ø. Fischer, Solid State Commun. 23, 37 (1977) 
205. J.W. Lynn, G. Shirane, W. Thomlinson, R.N. Shelton, Phys. Rev. Lett. 46, 368 (1981)

206. M.B. Maple, R.E. Baumbach, J.J. Hamlin, D.A. Zocco, B.J. Taylor, N.P. Butch, J.R. Jeffries, S.T. Weir, B.C. Sales, D. Mandrus, M.A. McGuire, A.S. Sefat, R. Jin, Y.K. Vohra, J.-H. Chu, I.R. Fisher, Physica B 404, 2924 (2009)

207. S.S. Saxena, P. Agarwal, K. Ahilan, F.M. Grosche, R.K. Haselwimmer, M. Steiner, E. Pugh, I.R. Walker, S.R. Julian, P. Monthoux, G.G. Lonzarich, A.D. Huxley, I. Sheikin, D. Braithwaite, J. Flouquet, Nature (London) 406, 587 (2000)

208. T. Akazawa, H. Hidaka, H. Kotegawa, T.C. Kobayashi, T. Fujiwara, E. Yamamoto, Y. Haga, R. Settai, Y. Ōnuki, J. Phys. Soc. Jpn. 73(11), 3129 (2004)

209. T. Akazawa, H. Hidaka, T. Fujiwara, T.C. Kobayashi, E. Yamamoto, Y. Haga, R. Settai, Y. Ōnuki, J. Phys., Condens. Matter 16, L29 (2004)

210. N.T. Huy, A. Gasparini, D.E. de Nijs, Y. Huang, J.C.P. Klaasse, T. Gortenmulder, A. de Visser, A. Hamann, T. Görlach, H. v. Löhneysen, Phys. Rev. Lett. 99, 067006 (2007)

211. N.F. Berk, J.R. Schrieffer, Phys. Rev. Lett. 17, 433 (1966)

212. D.E. de Nijs, N.T. Huy, A. de Visser, Phys. Rev. B 77, 140506 (2008)

213. N.T. Huy, A. de Visser, Solid State Commun. 149, 703 (2009)

214. D. Belitz, T.R. Kirkpatrick, T. Vojta, Phys. Rev. Lett. 82(23), 4707 (1999)

215. C. Pfleiderer, J. Phys., Condens. Matter 17, S987 (2005)

216. C. Pfleiderer, A.D. Huxley, Phys. Rev. Lett. 89, 147005 (2002)

217. I. Sheikin, A. Huxley, D. Braithwaite, J.P. Brison, S. Watanabe, K. Miyake, J. Flouquet, Phys. Rev. B 64, 220503(R) (2001)

218. G. Oomi, T. Kagayama, Y. Ōnuki, J. Alloys Compd. 271, 482 (1998)

219. N. Tateiwa, T.C. Kobayashi, K. Hanazono, K. Amaya, Y. Haga, R. Settai, Y. Ōnuki, J. Phys., Condens. Matter 13, L17 (2001)

220. R. Vollmer, C. Pfleiderer, H. v. Löhneysen, E.D. Bauer, M.B. Maple, Physica B 312-313, 112 (2002)

221. K. Shimizu, T. Kimura, S. Furomoto, K. Takeda, K. Kontani, Y. Onuki, K. Amaya, Nature 412, 316 (2001)

222. O. Mathon, F. Baudelet, J.P. Itié, A. Polian, M. d'Astuto, J.C. Chervin, S. Pascarelli, Phys. Rev. Lett. 93, 255503 (2004)

223. T. Moriya, Spin Fluctuations in Itinerant Electron Magnetism (Springer, Berlin, 1985)

224. A. Miyake, D. Aoki, J. Flouquet, J. Phys. Soc. Jpn. 77, 094709 (2008)

225. A.D. Huxley, S.J.C. Yates, F. Lévy, I. Sheikin, J. Phys. Soc. Jpn. 76, 051011 (2007)

226. T.C. Kobayashi, A. Hori, S. Fukushima, H. Hidaka, H. Kotegawa, T. Akazawa, K. Takeda, Y. Ohishi, E. Yamamoto, J. Phys. Soc. Jpn. 76, 051007 (2007)

227. E.D. Bauer, E.J. Freeman, C. Sirvent, M.B. Maple, J. Phys., Condens. Matter 13, 5675 (2001)

228. T.C. Kobayashi, S. Fukushima, H. Hidaka, K. Kotegawa, T. Akazawa, E. Yamamoto, Y. Haga, R. Settai, Y. Ōnuki, Physica B 378, 355 (2006)

229. J. Linder, A.H. Nevidomskyy, A. Sudbø, Phys. Rev. B 78, 172502 (2008)

230. T. Ohta, T. Hattori, K. Ishida, Y. Nakai, E. Osaki, K. Deguchi, N.K. Sato, I. Satoh, J. Phys. Soc. Jpn. 79(2), 023707 (2010)

231. D. Aoki, T.D. Matsuda, V. Taufour, E. Hassinger, G. Knebel, J. Flouquet, J. Phys. Soc. Jpn. 78(11), 113709 (2009)

232. J. Pospíšil, J.P. Vejpravová, M. Diviš, V. Sechovský, J. Appl. Phys. 105, 07E114 (2009)

233. manuscript in preparation

234. J.J. Hamlin, R.E. Baumbach, K. Huang, M. Janoschek, N. Kanchanavatee, D.A. Zocco, M.B. Maple, Proc. Mat. Res. Soc. (2010, accepted) doi:10.1557/PROC-1264-Z12-04

235. P. Gegenwart, J. Custers, C. Geibel, K. Neumaier, T. Tayama, K. Tenya, O. Trovarelli, F. Steglich, Phys. Rev. Lett. 89, 056402 (2002)

236. H. v. Löhneysen, A. Rosch, M. Vojta, P. Wölfle, Rev. Mod. Phys. 79, 1015 (2007)

237. P. Gegenwart, J. Custers, Y. Tokiwa, C. Geibel, F. Steglich, Phys. Rev. Lett. 94, 076402 (2005)

238. J. Custers, P. Gegenwart, H. Wilhelm, K. Neumaier, Y. Tokiwa, O. Trovarelli, C. Geibel, F. Steglich, C. Pépin, P. Coleman, Nature 424, 524 (2003)

239. O. Stockert, M.M. Koza, J. Ferstl, A.P. Murani, C. Geibel, F. Steglich, Physica B 378, 157 (2006)

240. R. Küchler, N. Oeschler, P. Gegenwart, T. Cichorek, K. Neumaier, O. Tegus, C. Geibel, J.A. Mydosh, F. Steglich, L. Zhu, Q. Si, Phys. Rev. Lett. 91, 066405 (2003)

241. K. Ishida, K. Okamoto, Y. Kawasaki, Y. Kitaoka, O. Trovarelli, C. Geibel, F. Steglich, Phys. Rev. Lett. 89, 107202 (2002)

242. J. Sichelschmidt, V.A. Ivanshin, J. Ferstl, C. Geibel, F. Steglich, Phys. Rev. Lett. 91, 156401 (2003) 
243. S. Paschen, T. Lühmann, S. Wirth, P. Gegenwart, O. Trovarelli, C. Geibel, F. Steglich, P. Coleman, Q. Si, Nature 432, 881 (2004)

244. S. Hartmann, N. Oeschler, C. Krellner, C. Geibel, S. Paschen, F. Steglich, Phys. Rev. Lett. 104, 096401 (2010)

245. P. Gegenwart, Q. Si, F. Steglich, Nat. Phys. 4, 186 (2008)

246. R.E. Baumbach, J.J. Hamlin, L. Shu, D.A. Zocco, J.R. O’Brien, P.-C. Ho, M.B. Maple, Phys. Rev. Lett. (2010, accepted)

247. W. Jeitschko, D.J. Braun, R.H. Ashcraft, R. Marchand, J. Solid State Chem. 25, 309 (1978)

248. Y.M. Prots, W. Jeitschko, Inorg. Chem. 37, 5431 (1998)

249. B.C. Sales, Handbook on the Physics and Chemistry of the Rare Earths, vol. 33 (Elsevier, Amsterdam, 2002)

250. M.B. Maple, Z. Henkie, R.E. Baumbach, T.A. Sayles, N.P. Butch, P.-C. Ho, T. Yanagisawa, W.M. Yuhasz, R. Wawryk, T. Cichorek, A. Pietraszko, J. Phys. Soc. Jpn. 77(Suppl. A), 7 (2008)

251. H. Sato, D. Kikuchi, K. Tanaka, M. Ueda, H. Aoki, T. Ikeno, S. Tatsuoka, K. Kuwahara, Y. Aoki, M. Koghi, H. Sugawara, K. Iwasa, H. Harima, J. Phys. Soc. Jpn. 77(Suppl. A), 1 (2008)

252. Y. Aoki, A. Tsuchiya, T. Kanayama, S.R. Saha, H. Sugawara, H. Sato, W. Higemoto, A. Koda, K. Ohishi, K. Nishiyama, R. Kadono, Phys. Rev. Lett. 91, 067003 (2003)

253. K. Izawa, Y. Nakajima, J. Goryo, Y. Matsuda, S. Osaki, H. Sugawara, H. Sato, P. Thalmeier, K. Maki, Phys. Rev. Lett. 90, 117001 (2003)

254. R.W. Hill, S. Li, M.B. Maple, L. Taillefer, Phys. Rev. Lett. 101, 237005 (2008)

255. E.E.M. Chia, M.B. Salamon, H. Sugawara, H. Sato, Phys. Rev. Lett. 91, 247003 (2003)

256. G. Seyfarth, J.P. Brison, M.A. Méasson, D. Braithwaite, G. Lapertot, J. Flouquet, Phys. Rev. Lett. 97, 236403 (2006)

257. D.E. MacLaughlin, L. Shu, R.H. Heffner, J.E. Sonier, F.D. Callaghan, G.D. Morris, O.O. Bernal, W.M. Yuhasz, N.A. Frederick, M.B. Maple, Physica B 403, 1132 (2008)

258. L. Shu, D.E. MacLaughlin, W.P. Beyermann, R.H. Heffner, G.D. Morris, O.O. Bernal, F.D. Callaghan, J.E. Sonier, W.M. Yuhasz, N.A. Frederick, M.B. Maple, Phys. Rev. B 79, 174511 (2009)

259. S. Rahimi, M.B. Maple, R.W. Hill, J. Phys., Condens. Matter 20, 215209 (2008)

260. P.-C. Ho, N.A. Frederick, V.S. Zapf, E.D. Bauer, T.D. Do, M.B. Maple, A. Christianson, A.H. Lacerda, Phys. Rev. B 67, 180508(R) (2003)

261. M. Kohgi, K. Iwasa, M. Nakajima, N. Metoki, S. Araki, N. Bernhoeft, J.M. Mignot, A. Gukasov, H. Sato, Y. Aoki, H. Sugawara, J. Phys. Soc. Jpn. 72, 1002 (2003)

262. C.S. Turel, J.Y.T. Wei, W.M. Yuhasz, R.E. Baumbach, M.B. Maple, J. Phys. Soc. Jpn. 77(Suppl. A), $21(2008)$

263. T. Cichorek, A.C. Mota, F. Steglich, N.A. Frederick, W.M. Yuhasz, M.B. Maple, Phys. Rev. Lett. 94, 107002 (2005)

264. E.E.M. Chia, D. Vandervelde, M.B. Salamon, D. Kikuchi, H. Sugawara, H. Sato, J. Phys., Condens. Matter 17, L303 (2005)

265. P.-C. Ho, W.M. Yuhasz, N.P. Butch, N.A. Frederick, T.A. Sayles, J.R. Jeffries, M.B. Maple, J.B. Betts, A.H. Lacerda, Phys. Rev. B 72, 094410 (2005)

266. N.A. Frederick, T.D. Do, P.-C. Ho, N.P. Butch, V.S. Zapf, M.B. Maple, Phys. Rev. B 69, 024523 (2004)

267. P.-C. Ho, T. Yanagisawa, N.P. Butch, W.M. Yuhasz, C.C. Robinson, A.A. Dooraghi, M.B. Maple, Physica B 403, 1038 (2008)

268. N.A. Frederick, T.A. Sayles, M.B. Maple, Phys. Rev. B 71, 064508 (2005)

269. N.A. Frederick, T.A. Sayles, S.K. Kim, M.B. Maple, J. Low Temp. Phys. 147, 321 (2007)

270. P.-C. Ho, N.P. Butch, V.S. Zapf, T. Yanagisawa, N.A. Frederick, S.K. Kim, W.M. Yuhasz, M.B. Maple, J.B. Betts, A.H. Lacerda, J. Phys., Condens. Matter 20, 215226 (2008) 